\title{
Prediction of Noise Levels and Annoyance from Aircraft Run-ups at the Vancouver International Airport
}

by

\author{
Katrina Anna Scherebnyj \\ B.Sc.Eng., Queen's University, 2004 \\ A THESIS SUBMITTED IN PARTIAL FULFILMENT OF \\ THE REQUIREMENTS FOR THE DEGREE OF \\ Master of Applied Science \\ in \\ The Faculty of Graduate Studies \\ (Mechanical Engineering)
}

The University of British Columbia

September 2006

(C) Katrina Anna Scherebnyj 2006 


\section{Abstract}

The Vancouver International Airport Authority (YVRAA), responsible for managing the airport, has been receiving an ever-increasing number of noise complaints resulting from engine run-ups. Engine run-ups - the testing of stationary aircraft's engines following maintenance - often take place at night in order to promptly return aircraft into service the following morning, thus causing noise disturbance in nearby communities. There are certain run-up parameters - such as the location and heading of the aircraft - which the airport can control. A model to predict noise levels, from which annoyance can be inferred and which YVRAA can use to manage run-ups, has been developed. A basic 2D, single-frequency version of the Green's Function Parabolic Equation (GFPE) model for predicting outdoor noise levels was modified to include spatially-varying types of ground, turbulence, and air absorption. Sound-speed profiles resulting from wind and temperature gradients were also added, and the model was extended to predict 1/3-octave-band levels. The sound pressure levels and directivities of a small jet aircraft, the CRJ200, were measured in order to characterize its noise radiation. These and other data were used as the model input source data. The model modifications were evaluated by comparison with literature; predictions agreed well, typically within a couple of $d B$. The GFPE model was then used to calculate the transmission loss from run-up pads to community receiver locations, and corresponding annoyance levels, for various atmospheric conditions. A sensitivity analysis found the GFPE model to be most sensitive to wind, the state of the atmosphere, and the aircraft power setting and heading. Predictions for 17 run-ups were made; results were within $10 \mathrm{dBA}$ in cases for which all input information was available, and within $20 \mathrm{dBA}$ when some input data was unavailable. The percentage of people annoyed, as indicated by the percentage of people awoken from sleep, was predicted from the noise level predictions to be between 1.8 and $9.5 \%$. 


\section{Contents}

Abstract ................................

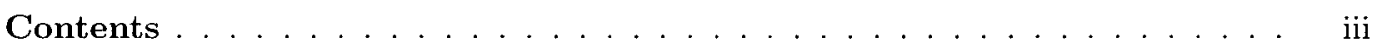

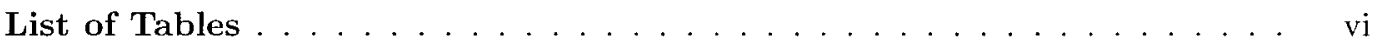

List of Figures . . . . . . . . . . . . . . . . . . . vii

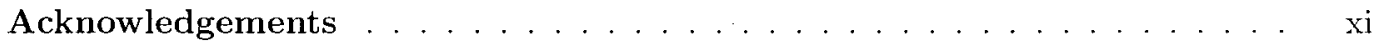

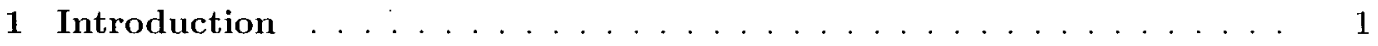

1.1 The Vancouver International Airport $(Y V R) \ldots \ldots \ldots$

1.2 Basics of Outdoor Sound . . . . . . . . . . . . . . . . . 5

1.2.1 Basic Acoustic Theory . . . . . . . . . . . . . . . . . . . 6

1.2.2 Sources and Propagation .................. 8

1.2 .3 Ground Impedance . . . . . . . . . . . . . . . . . . . . . . 11

1.2.4 Atmospheric Absorption . . . . . . . . . . . . . . . 15

1.2 .5 Inhomogeneous Atmosphere . . . . . . . . . . . . . 16

1.2 .6 Turbulence . . . . . . . . . . . . . . . . . . . . . . . . 17

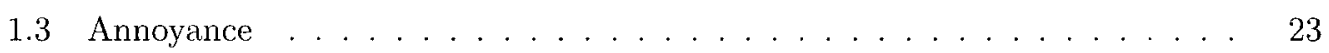

1.3.1 Annoyance and Sleep Disturbance ... . . . . . . . . . . 23

1.3.2 Quantification ........................ 24

1.3.3 Relationships . . . . . . . . . . . . . . . . . 26

1.4 Research Objectives . . . . . . . . . . . . . . . . . . . 28

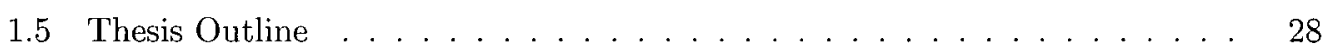

2 Run-up Noise Measurements . . . . . . . . . . . . . . . . . . . . . . . . . . 29

2.1 Introduction . . . . . . . . . . . . . . . . . . . . . . 29 
2.2 Meteorological Conditions . . . . . . . . . . . . . . . . . . . . 30

2.3 Equipment . . . . . . . . . . . . . . . . . . . . . 31

2.4 Experimental Procedure $\ldots \ldots \ldots \ldots \ldots \ldots \ldots \ldots$

2.4 .1 Calibration . . . . . . . . . . . . . 33

2.4 .2 Procedure . . . . . . . . . . . . . . . 33

2.5 Results. . . . . . . . . . . . . . . . . . . 34

2.6 Conclusions . . . . . . . . . . . . . . . . . . . . . . . . 39

3 Outdoor Sound Propagation Modeling and the GFPE Model . . . . 40

3.1 Introduction to Models . . . . . . . . . . . . . . . . . . . . 40

3.2 The Original GFPE Code and Required Modifications . . . . . . . . . . 41

3.3 GFPE Theory . . . . . . . . . . . . . . . . . . . . 42

3.3 .1 Origins of Sound Equations . . . . . . . . . . . 43

3.3 .2 The Helmholtz Equation . . . . . . . . . . . . . . . . . . 44

3.3.3 A more 'realistic' atmosphere: vertical sound-speed profiles . . . . . 44

3.3 .4 Refraction . . . . . . . . . . . . . . . . . 48

3.3 .5 Starting Field . . . . . . . . . . . . . . . . . 49

3.3 .6 Elevation Angle Limitation . . . . . . . . . . . . . . 50

3.4 Modifications to GFPE . . . . . . . . . . . . . . . . . . 50

3.4 .1 Variable Grounds . . . . . . . . . . . . . . . . . . 51

3.4 .2 Step Size . . . . . . . . . . . . . . . . . . . . . 52

3.4 .3 Multiple Frequencies . . . . . . . . . . . . . . 53

3.4 .4 Corrections: A-Weighting; Air Absorption . . . . . . . . . . 53

3.4 .5 Turbulence . . . . . . . . . . . . . . . . . . 55

3.4 .6 Sound Speed Profile . . . . . . . . . . . . . . . . 57

3.4 .7 Sound Exposure Level (SEL) _ . . . . . . . . . . . . . 58

4 GFPE Model Evaluation . . . . . . . . . . . . . . . . 59

4.1 Homogeneous Atmosphere (No Refraction) . . . . . . . . . . . . . 59

4.2 Downward and Upward Refracting Atmospheres . . . . . . . . . . . . 61

4.3 Mixed Ground Impedance . . . . . . . . . . . . . . . . . . 64

4.4 Turbulence . . . . . . . . . . . . . . . 67

4.5 Summary . . . . . . . . . . . . . . . . . . 71 
5 Run-up Noise and Annoyance Predictions . . . . . . . . . . . 72

5.1 Atmospheric Predictions . . . . . . . . . . . . . . . . 73

5.2 Prediction Uncertainty . . . . . . . . . . . . . 75

5.2 .1 Summary . . . . . . . . . . . . . . . . . 80

5.3 GFPE Run-up Prediction Results . . . . . . . . . . . . . . . . . 80

5.3.1 Available Aircraft Source Levels, Known Headings . . . . . . . . . 84

5.3.2 Unavailable Aircraft Source Levels, Known Headings . . . . . . . . 85

5.3.3 Available Aircraft Source Levels, Unknown Headings . . . . . . . . 85

5.3.4 Unavailable Aircraft Source Levels, Unknown Headings . . . . . . 86

5.3.5 Predictions With Winds Above $5 \mathrm{~m} / \mathrm{s} \ldots \ldots \ldots$. . . . . 87

5.3 .6 Summary . . . . . . . . . . . . . . . . . 87

5.4 SEL and Annoyance . . . . . . . . . . . . . . . . 90

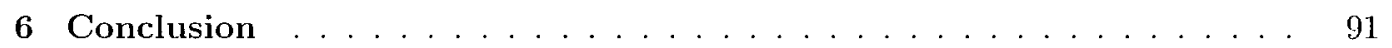

6.1 Summary . . . . . . . . . . . . . . . . . . 91

6.2 Contribution to the field . . . . . . . . . . . . . . . . . 93

6.3 Future Work . . . . . . . . . . . . . . . . . . . 93

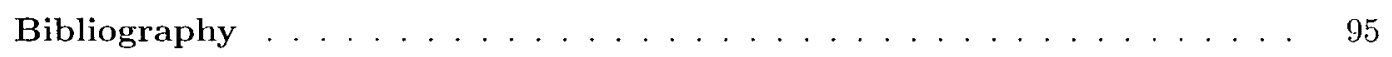




\section{List of Tables}

1.1 Complaints resulting from engine run-ups . . . . . . . . . . . . 2

2.1 Equipment used for measurements. . . . . . . . . . . . . . . . . 32

3.1 Parameters used for finite ground impedance calculations. 0.5 was used for the pore shape factor ratio in all cases $[1] \ldots \ldots \ldots 2$

3.2 A-weighting correction for $1 / 3$ octave bands, according to IEC 651 (1993-09)

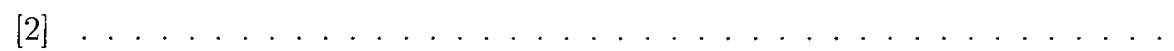

3.3 Attenuation in third-octave bands resulting from atmospheric absorption. Values are listed in $\mathrm{dB} / \mathrm{km}$, for temperature of $283.15 \mathrm{~K}$, relative humidity of $80 \%$, and atmospheric pressure of $1.01325 \times 10^{5} \mathrm{~Pa}[2] \ldots \ldots \ldots$

3.4 Comparison of attenuation [in $\mathrm{dB}$ ] resulting from a linear sound speed gradient at select frequencies and source-receiver distances . . . . . . . . . .

5.1 Summary of run-up measurement and prediction results for events from Jan. '05 to June '06. Values in () are full power levels, with idle levels in the same cell; values in \{\} are at Pos. 0 , with Pos. 100 in the same cell; and values in [] are full power at the correct, position, with values at idle, but at the incorrect positions, in the same cell. Cells highlighted ( $\mathbf{(})$ represent stable,

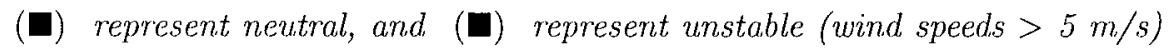
conditions.

5.2 Summary of run-up event categories, separated into those within $10 \mathrm{dBA}$ of the average, and those for which predictions were greater than $10 \mathrm{dBA}$ of the

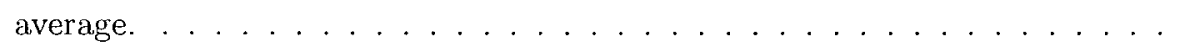

5.3 Summary of SEL data corresponding to SEL GFPE predictions . . . . . . . 


\section{List of Figures}

1.1 Aerial photograph of YVR $[3] . \ldots \ldots \ldots \ldots$

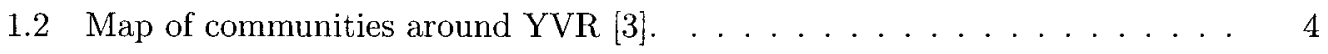

1.3 Run-up locations at YVR $[4] . \ldots \ldots \ldots \ldots \ldots$

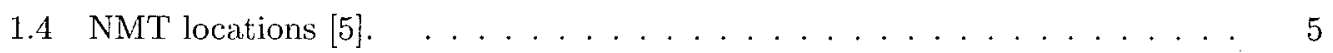

1.5 Geometrical spreading of a point, line, and planar source. . . . . . . 9

1.6 Reflection of a sound wave upon incidence $[6] \ldots \ldots . \ldots . \ldots 12$

1.7 Normalized specific acoustic impedance: Measured ( - . - ): curve E by Embleton et al.; curve D by Daigle and Stinson; and curve Z by Zuckerwar; and theory (-): curve A by Attenborough (5-parameter model); and curve C by Chessell (extension to Delany and Bazley's work) $[6]$. . . . . . . . 15

1.8 Wave refraction in downwind (similar to temperature inversion conditions) and in upwind (similar to temperature lapse conditions) [7] $\ldots \ldots \ldots 17$

1.9 Turbulence record of wind and temperature fluctuations (a) with time, and (b) with frequency $[8] . \ldots \ldots \ldots \ldots \ldots \ldots$

1.10 Relative sound-pressure level as a function of range in an upward-refracting atmosphere. Values here are typical of a strong upward refracting atmosphere $[9]$

1.11 Factors affecting annoyance according to FICAN $[10] \ldots \ldots \ldots . \ldots 24$

1.12 Comparison of FICAN and FICON sleep awakening relationships [11]. . . 27

1.13 Prevalence of awakening in laboratory and field environments based on indoor sound exposure level $[12] \ldots \ldots \ldots \ldots$. . . . . . . . . . . . . . . 27

2.1 The CRJ200 aircraft. . . . . . . . . . . . . . . . . . 30

2.2 Background levels at the run-up site prior to run-up. . . . . . . . . 31

2.3 Measurements locations for noise measurements. . . . . . . . . . . . . . . 32 
2.4 Comparison of directivity symmetry of CRJ200. Solid colors are for the right hand side, hatched colors for the corresponding left hand side of the aircraft. 35

2.5 Variation with position of difference in $5 \mathrm{~s}$ Leq at two measurement heights. Plotted values are the $0 \mathrm{~m}$ level minus the $1.4 \mathrm{~m}$ level. (_.... ) idle power,

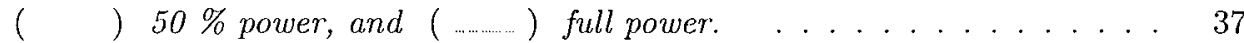

2.6 Dash $8 \mathrm{Lp}$ at $40 \mathrm{~m}$; degree positions correspond to those in Figure 2.3 [13]. 38

2.7 CRJ200 Lp at 40 m; degree positions correspond to those in Figure 2.3. . . 38

3.1 Gaussian starting function $[14] . \ldots \ldots \ldots$. . . . . . . . . . . 49

3.2 Elevation angle limitation: between angles $-\gamma_{\max }$ and $\gamma_{\max }$, the PE model is valid $[2] \ldots \ldots \ldots \ldots \ldots \ldots$

4.1 Transmission loss for the homogeneous atmosphere benchmark case. Input parameters include $h_{\text {source }}=5 \mathrm{~m}, \mathrm{~h}_{\text {receiver }}=1 \mathrm{~m}$, and normalized ground impedance for 10,100 , and $1000 \mathrm{~Hz}$ of $(38.79,38.41),(12.81,11.62)$, and $(5.96$, 2.46), respectively [15].

4.2 GFPE prediction corresponding to Figure 4.1. Parameters used in prediction include: FFT length $=16384$, vertical step size $=\lambda / 5$, vertical height $=250$ $\mathrm{m}$, speed of sound $=343 \mathrm{~m} / \mathrm{s}$, surface wave integration number $250 . \ldots$.

4.3 Transmission loss for the downward refracting atmosphere from benchmark paper. Similar input parameters as Figure 4.1, but with sound speed gradient

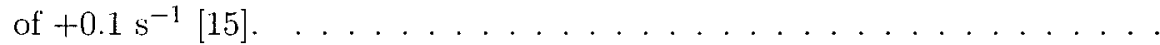

4.4 GFPE prediction corresponding to Figure 4.3. Similar input parameters as Figure 4.2 , but with sound speed gradient of $+0.1 \mathrm{~s}^{-1} \ldots \ldots \ldots 62$

4.5 Transmission loss for the upward refracting atmosphere benchmark case. Similar input parameters as Figure 4.1, but with sound speed gradient of

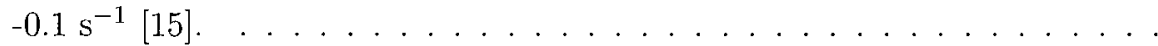

4.6 GFPE prediction corresponding to Figure 4.5. Similar input parameters as Figure 4.2 , but with sound speed gradient of $-0.1 \mathrm{~s}^{-1} \ldots \ldots \ldots \ldots$

4.7 Excess attenuation for a mixed impedance ground. The solid line is for the CNPE results, the dots for the Padé predictions, the diamonds are for the single impedance case $(160 \mathrm{HZ})[16] \ldots \ldots \ldots \ldots$ 
4.8 GFPE prediction corresponding to Figure 4.7. Parameters used in prediction include: $h_{\text {source }}=1.5 \mathrm{~m}$, FFT length $=16384, h_{\text {receiver }}=1.8 \mathrm{~m}, \Delta z=\lambda$ / $10, h_{\text {vertical }}=100 \mathrm{~m}, \mathrm{c}=340 \mathrm{~m} / \mathrm{s}$, Delany and Bazley model $\sigma_{1}=2 \cdot 10^{5} \mathrm{kPa}$, $\sigma_{2}=2 \cdot 10^{2} \mathrm{kPa}$ ), discontinuity at $50 \mathrm{~m}$; surface wave integration number = 100. $(\bigcirc)$ is the GFPE prediction, (......) is the hard impedance only prediction, and (......) is the soft impedance only prediction, and (-----) is the CNPE estimate from Figure $4.7 \ldots \ldots \ldots \ldots$

4.9 Comparison of calculated curves for two models across an impedance discontinuity: Rasmussen (_-----) and De Jong (_ _ _ ). The dotted curves are predictions for all hard or all soft ground (no discontinuity). $\mathrm{h}_{\text {source }}=1.5$ $\mathrm{m}, \mathrm{h}_{\text {receiver }}=1.5 \mathrm{~m}$, source-receiver distance $=5 \mathrm{~m}$, first portion of ground hard $\left(\sigma_{1}=30000 \mathrm{cgs}\right.$ rayls), second portion grass ( $\sigma_{2}=300 \mathrm{cgs}$ rayls $)[17]$.

4.10 GFPE prediction corresponding to Figure 4.9. ( $O$ ) is for GFPE predictions, and (_.....) is an estimate of Ramussen's model from Figure 4.9 for

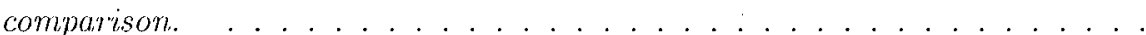

4.11 Relative sound pressure level prediction for two turbulence simulations using the CNPE at $424 \mathrm{~Hz}$. Joint dots are Weiner et al.'s measurements; lines are Gilbert et al.'s CNPE predictions. The root-mean-square fluctuation and correlation length are $1.42 \times 10^{-3}$ and $1.1 \mathrm{~m}$, respectively, $\mathrm{a}=-0.5 \mathrm{~m} / \mathrm{s}$ and $-2.0 \mathrm{~m} / \mathrm{s}$ for weak and strong refraction, respectively $\left(\mathrm{c}(\mathrm{z})=\mathrm{c}_{o}=\mathrm{a} \ln z / d\right)$, $\mathrm{d}=6 \times 10^{-3} \mathrm{~m}, \mathrm{Z}=8.00+\mathrm{i} 9.24$, source height $=1.8 \mathrm{~m}$, receiver height $=$ $1.5 \mathrm{~m}[9]$.

4.12 Relative sound pressure level prediction for two turbulence simulations using the CNPE at $848 \mathrm{~Hz}$. Joint dots are Weiner et al.'s measurements; lines are Gilbert et al.'s CNPE predictions. Same parameters as Figure 4.11, except $\mathrm{Z}=5.17+\mathrm{i} 5.57[9]$.

4.13 GFPE predictions with turbulence in a weak and strong upward refracting atmosphere. Parameters correspond to Figures 4.11 and 4.12 . (......) is the non-turbulent prediction, $(\ldots, \ldots),(\quad)$, and $(\ldots . . .$.$) correspond to the three$ predictions with turbulence, (_---) is the $d B$ average of the three turbulent predictions, and $(\bullet)$ are Weiner et al.'s measurements from Figures 4.11 and 4.12 
4.14 GFPE predictions with turbulence in a homogeneous (no refraction), weak, and strong downward refracting atmosphere. All other parameters are the same as Figures 4.11 and 4.12. (_...) is the non-turbulent prediction, (.....), ( ), and ( ...) correspond to the three predictions with turbulence, (----) is the $d B$ average of the three turbulent predictions, and $(\bullet)$ are'Weiner et al.'s measurements from Figures 4.11 and 4.12 for the weak upward refracting atmosphere.

5.1 Atmospheric stability test: run-ups in the upper left hand corner took place under stable conditions; run-ups in the lower left hand corner took place under neutral conditions. Strong winds make it difficult to predict the atmospheric conditions on the right hand side. $\ldots \ldots \ldots \ldots$

5.2 Reference test case compared with predictions for input variations in position (distance) and ground type. (.....) is the reference level. . . . . . . . . . 77

5.3 Reference test case (71.29 dBA) compared with predictions for input variations in wind, temperature, height of propagation, and aircraft details.

) is the reference level. . . . . . . . . . . . . .

5.4 Data from NMT 3, October 12, 2005. A noise event is apparent from 0:00 to $0: 20$ AM. $(\sim 0: 30$ to $0: 50$ on the time axis $) \ldots \ldots \ldots \ldots$

5.5 Summary of run-up noise levels from GFPE predictions, and estimates from NMT data. NMT Leq average, low GFPE prediction, high GFPE prediction; black lines indicate the range of NMT levels (from back-

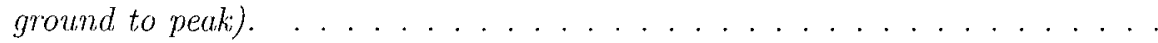

5.6 GFPE predictions using turbulence for the 8-Sept-05 sound speed profile. $(\ldots)$ is the non-turbulent prediction, $(\ldots . .),.(\quad)$, and $\left({ }_{-}\right.$.) correspond to the three predictions with turbulence, and (---) is the $d B$ average of the three turbulent predictions. . . . . . . . . . . . . . .

5.7 Scatter plot comparing GFPE predictions with NMT measurements. (....) represents $y=x$, and 10 and $20 \mathrm{dBA}$ offsets, ( $)$ are the GFPE predictions where some information was unknown, and are the GFPE predictions where all information was known. Error bars represent the background and peak NMT measurement levels. . . . . . . . . . . . . . . . . . . . 


\section{Acknowledgements}

To my supervisor at UBC, Dr. Murray Hodgson, thank you. Your ongoing support and advice with my thesis research, school courses, and future acoustics ambitions has been invaluable. You've pushed me to work to my best abilities over the last two years, and I have been rewarded with successful research, a broad exposure to the acoustical world, and above all else, a sound acoustics background.

To Mark Cheng and Fred Tewfik, and the rest of Vancouver Airport Authority, your eagerness to help in anyway possible was invaluable. Thank you for your all technical support. I enjoyed the time I spent working with you all at YVRAA. Devin, thank you for your help organizing the engine run-up; and Trudi, thank you and Jeff for coming out (both evenings!). That night couldn't have happened without Air Canada Jazz, who graciously provided a CR,J200 to be run-up, however I wanted. That was quite a feeling! To the people in the lab who worked overtime the night of the engine run-up measurements - thank you Gary, Natalie, Rimi, and Vincent, for all your help. Hopefully no more engines will fail the night of future planned run-ups!

To everyone in the Acoustics Lab - who is there now, and who has worked there in the last two years - you have all helped broaden my knowledge not only of acoustics, but of the world. You all helped bring a little light to a windowless room! Zohreh, thank you for all the chats and lunch time swims; and Galen, thank you for all your help and advice, even after you finished.

I have been lucky throughout the last two years to have received the help from many experts in acoustics, and other fields relating to this project. I'd like to thank Dr. Kenneth Gilbert and Dr. Xiao Di, who developed the GFPE code, for helping with my many coderelated questions; Dr. Keith Attenborough, for his help applying ground impedance models; Dr. Gilles Daigle and Dr. Mike Stinson, who helped take the disorder out of turbulence; Dr. Karl Pearsons, for helping me find a way to quantify annoyance; and Dr. Douw Steyn, who helped me understand the states of the atmosphere, so I could model its affects on the 
speed of sound.

Thank you to my family, who have forever been compassionate and encouraging, and especially patient, of everything I do. Mom and Dad, you have shown me by example how far hard work can go. I appreciate your love and support more than words can say. Tasha, you've always helped me keep life in perspective, and make time for fun. And to little Ava, with your irresistible little smile, no one can brighten a gloomy day quite like you. Kevin, thank you for your sagacious confidence, your plethora of ideas - you are a rara avis - but most of all, thank you for being my friend and escaping with me in the mountains. On whichever continent they may be.

And to you, thank you for picking up my master thesis and having a read through. It gets more exciting with each turn of the page, so don't stop until you've reached the end!

Katrina Scherebnyj,

August, 2006. 


\section{Chapter 1}

\section{Introduction}

The body that manages the Vancouver International Airport, the Vancouver International Airport Authority (YVRAA), receives hundreds of noise complaints each year. The causes of these complaints include landings, takeoffs, overhead flights, and engine run-ups. Each of these activities can, to an extent, be controlled. A run-up, or the testing of a stationary aircraft's engines, is a routine maintenance procedure that occurs following repairs to the aircraft. The number of complaints resulting specifically from engine run-ups has increased consistently over the last few years. For engine run-ups, there are certain parameters that affect community noise levels, such as the location and heading of the aircraft during the run-up, which the airport can manage. Following previous successful noise-related collaborative projects between YVRAA and the University of British Columbia (UBC) [13, 18], YVRAA and UBC agreed to collaborate on a new research project to predict annoyance in the communities surrounding the airport resulting from aircraft run-up noise.

The number of complaints received by YVR resulting from engine run-ups has been rising for several years now, as illustrated in Table 1.1. While there are some good reasons for this (such as increased traffic), engine run-l.ps are premeditated events and can be planned (time, location, olientation) to minimize noise disturbance in communities. The Airport Authority at YVR continuously strives to maintain good relations with its neighbours; this project was conceived to study the conditions for which engine run-ups will result in high noise levels in nearby communities, and provide a tool that can be used to mitigate noise by understanding the effects of atmospheric conditions on the propagation of noise.

The propagation of noise outdoors is a complex phenomenon. As sound propagates, it can be reflected, diffracted, attenuated, and amplified. Many factors influence the propagation of sound outdoors; the commonly assumed free-field conditions (only the direct wave and air absorption exist) over an infinite plane are over-simplified in the real world. For instance, acoustically hard surfaces such as concrete reflect a large portion of sound energy, 
Table 1.1: Complaints resulting from engine run-ups

\begin{tabular}{|c|c|c|}
\hline Year & $\begin{array}{c}\text { Number of run-up } \\
\text { complaints }\end{array}$ & $\begin{array}{c}\text { Percentage of total } \\
\text { noise complaints }\end{array}$ \\
\hline 2003 & 80 & $18 \%$ \\
\hline 2004 & 95 & $12 \%$ \\
\hline 2005 & 117 & $27 \%$ \\
\hline 2006 (until June 30) & 41 & $46 \%$ \\
\hline
\end{tabular}

creating significantly louder noise levels compared to acoustically soft surfaces, like grass fields and forest floors. Temperature profiles, such as inversions (increase in temperature with height) alter the speed of a part of a propagating sound wavefront in such a way that sound is diffracted upward, creating quiet zones at the ground level. Temperature and humidity are also important, as they affect, air absorption. These factors must all be considered when modeling sound outdoors.

This work extends one of the earlier UBC/YVRAA collaborative research projects in which a Green's Function Parablic Equation model (GFPE) was used in simple atmospheric conditions to investigate the feasibility of Active Noise Control (ANC - reducing noise with the introduction of additional noise) [13]. In this thesis, ANC is no longer considered, but the GFPE model is extended further.

In an effort to maintain good relations with the communities near the airport, YVRAA decided to look into developing a tool that could help determine under what conditions run-ups are expected to cause annoyance for the residents living nearby the airport. The objective of this work is to use additional environmental conditions - such as realistic wind and temperature profiles, mixed ground impedance, and atmospheric turbulence - to extend an existing sound propagation model to predict noise levels, and thus the community reaction in the residential areas surrounding the Vancouver Airport, for propeller and jet aircraft engine run-ups, in order to minimize community annoyance for given atmospheric conditions and airline requirements.

This thesis has been funded by the Natural Science and Engineering Council of Canada (NSERC) and YVRAA. 


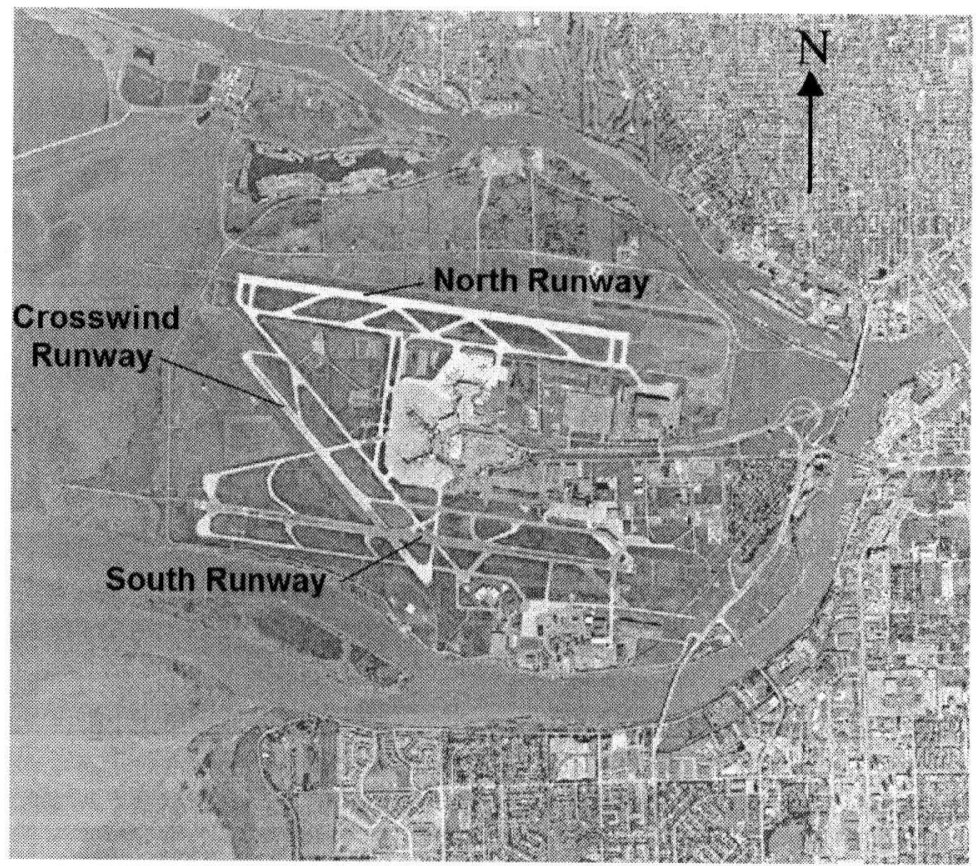

Figure 1.1: Aerial photograph of YVR [3].

\subsection{The Vancouver International Airport (YVR)}

The Vancouver International Airport (YVR) is a major gateway to Asia: it is the shortest flying distance between many Asian cities and North America, making it a premier destination for many airlines. It is also a regional hub, accessing small towns and communities throughout BC. As a result, unlike most other international airports, YVR has the unique feature that half of the aircraft activities result from jet aircraft, and the other half from propeller aircraft. YVR has three runways, as shown in Figure 1.1: the North runway, the South runway, and the Crosswind runway. The majority of winds at YVR come from the East or West; the runway locations allow the airplanes to face into the wind during landing and take off, as required. The Crosswind runway is used when the winds are from the Northwest or Southeast.

Vancouver is quite fortunate in its geographical location: the ocean on its West side allows landings and take-offs to take place over the water (wind permitting), minimizing the number of aircraft flying directly over the city. While certain areas, such as North Delta, located along the flight paths, may be of primary concern for overhead aircraft, run-ups typically disturb the communities closest to the airport (see Figure 1.2). This suggests that 


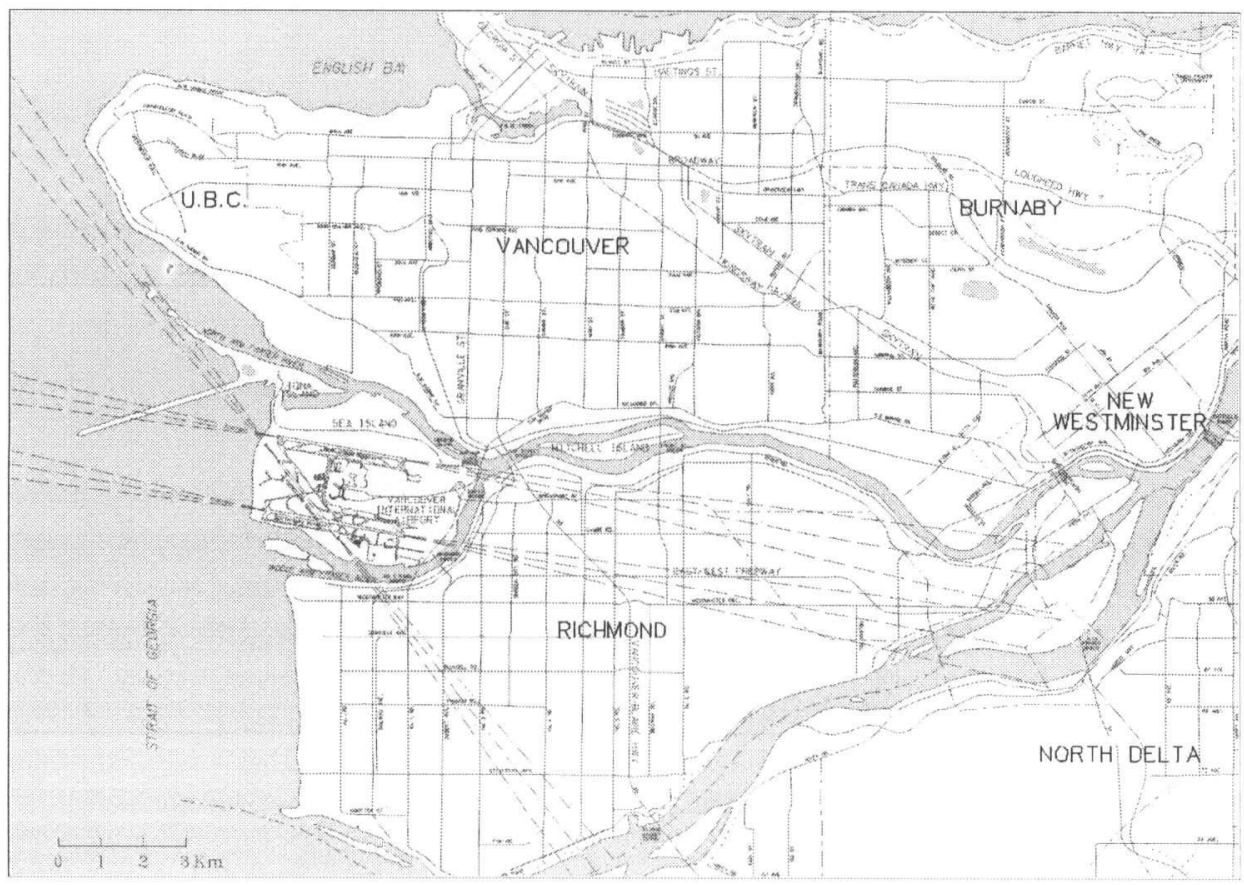

Figure 1.2: Map of communities around YVR [3].

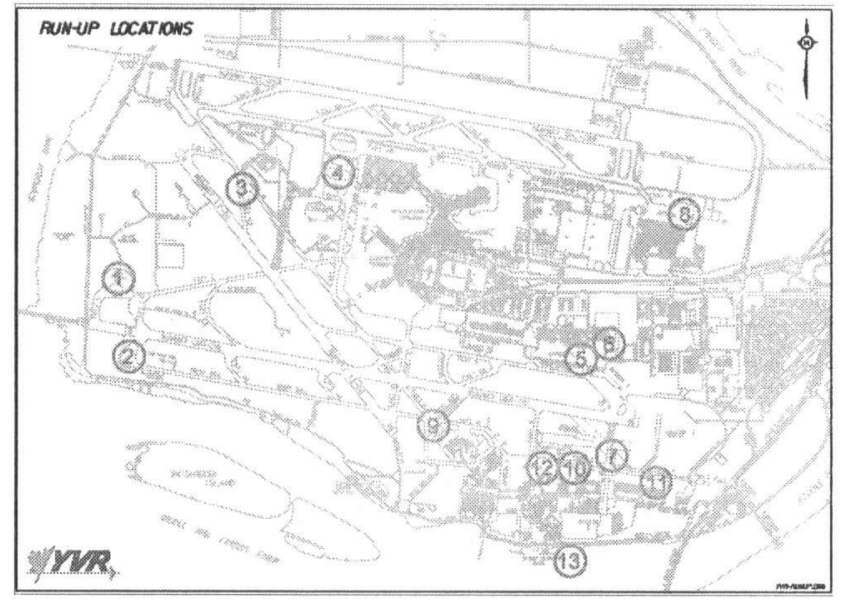

Figure 1.3: Run-up locations at YVR [4].

southern Vancouver and northern Richmond are the areas of concern for of this work.

Engine run-ups take place at designated run-ups pads. These are large, open areas, free of obstacles and accessible to aircraft via taxiways. The grounds are typically concrete, an 'acoustically hard' material. There are many run-up pads at the Vancouver Airport; the thirteen most common locations are illustrated in Figure 1.3. Run-up Pad Number 1 


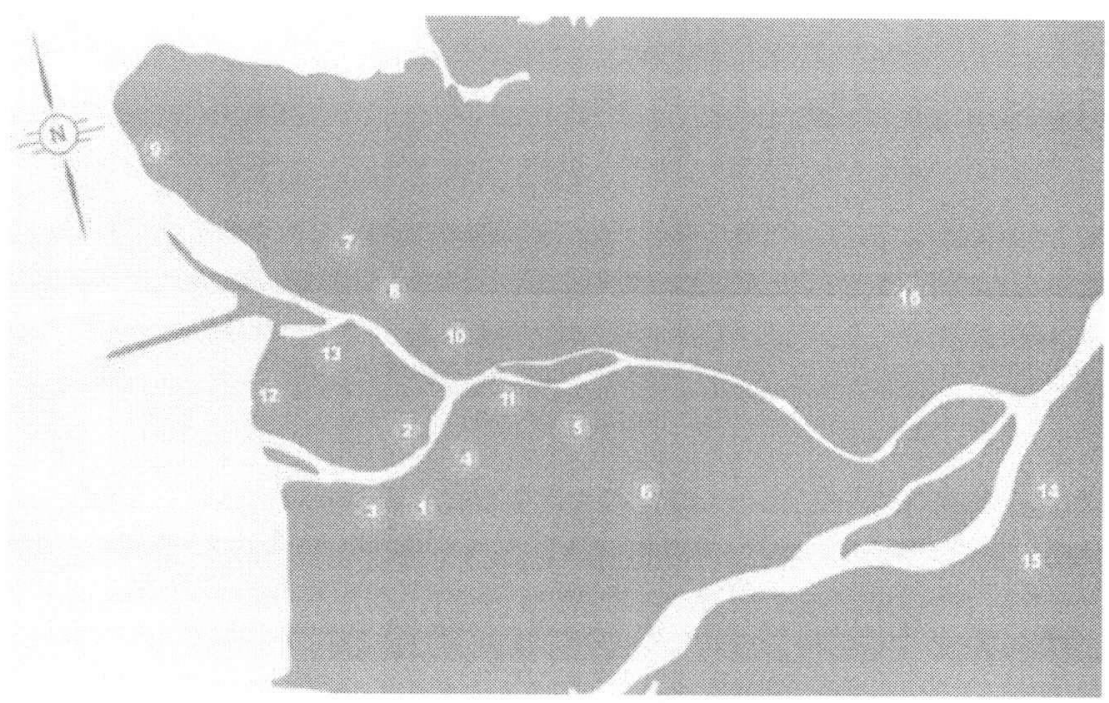

Figure 1.4: NMT locations [5].

was recently constructed in combination with the extension of the South runway. It, along with the run-up pad at the South Terminal (not shown, but close to Run-up Pad Number 13) and Run-up Pad Number 5 (Apron V), are often used for run-ups. Historically many complaints have resulted from activities in these three locations. An interesting feature of Apron $\mathrm{V}$ is the blast fence, located at the Southern end. The purpose of this barrier is to deflect and absorb sound propagating it its direction. A previous UBC student measured the insertion loss (energy loss through a barrier) of this particular blast fence [18].

In its efforts to manage noise, YVRAA has installed 16 noise monitoring terminals (NMTs) at and around the airport (see Figure 1.4). NMTs consist of microphones on poles, which measure A-weighted, 1-second $L_{e q}$ levels.

\subsection{Basics of Outdoor Sound}

Outdoor sound propagation governs noise levels at local communities resulting from runup activities. Outdoor sound propagation is highly dependent on meteorology and on the ground type $[6,19,20,21,22]$; both parameters are examined in detail in this work. Meteorological conditions that affect noise include wind speed and direction, temperature, wind and temperature gradients, humidity, and turbulence. Effects include changes in attenuation and directivity $[2,6,18]$. The type of ground surface is another important 
factor governing outdoor sound propagation: the ground impedance regulates the phase and magnitude of the reflected wave and, thus, the interaction of direct and reflected waves [19].

\subsubsection{Basic Acoustic Theory}

Sound - what we hear and feel - is the result of very rapid fluctuations in atmospheric pressure. Our ears are extremely sensitive organs, capable of differentiating fluctuations from $20 \mu \mathrm{Pa}$ to $100 \mathrm{~Pa}$, a range spanning many orders of magnitude. As a result, sound is typically qualified in decibels, a logarithmic unit. Sound pressures are converted to sound pressure levels, $L_{p}$, in decibels, as follows:

$$
L_{p}=20 \log \left(\frac{p}{p_{o}}\right)
$$

in $\mathrm{dB}$, where $p_{o}$ is a reference pressure. Here, $p_{o}$ is defined as the smallest pressure fluctu-

ation a normal hearing person can hear: $2 \times 10^{-5} \mathrm{~Pa}$ in air. The pressure, $p$, received at a listener's position, is a function of the source power and the distance from the source. The smallest change in decibel level people can usually hear is $\sim 1 \mathrm{~dB}$.

For a point source whose pressure fluctuations are radiating spherically, the pressure squared can be expressed as:

$$
p^{2}(r)=\frac{\rho c W}{4 \pi r^{2}}
$$

where $W$ (in Watts) is the sound power of the source, $c$ is the speed of sound (in $\mathrm{m} / \mathrm{s}$ ), $\rho$ is the density of the medium, and is $r$ the distance from the source (in $\mathrm{m}$ ). Pressure squared is proportional to energy; thus, according to Equation (1.1), an increase of $10 \mathrm{~dB}$ corresponds to a ten-fold increase in energy. This rapid increase in energy with decibel level is a serious concern when dealing with people and noise levels.

A healthy, normal-hearing person can typically hear sound in the frequency range of $20 \mathrm{~Hz}-20 \mathrm{kHz}$. This range decreases with noise exposure and age; elderly people, for instance, may only hear up to $14 \mathrm{kHz}$. Due to the geometry and size of the human ear, the ear does not respond linearly to these frequencies. For example, a $100 \mathrm{~Hz}, 60 \mathrm{~dB}$ pure tone will sound much quieter than a $1000 \mathrm{~Hz}, 60 \mathrm{~dB}$ pure tone (the human ear would perceive the $100 \mathrm{~Hz}$ tone to have the loudness of a $41 \mathrm{~dB}, 1000 \mathrm{~Hz}$ tone). This phenomena is quantified in acoustics as the 'A-weighting'. Sound pressure levels, $L_{p}$, can be quantified in the unweighted $\mathrm{dB}$ as described in Equation (1.1), and also by the A-weighted $\mathrm{dB}$, in 
$\mathrm{dBA}$, where a correction based on an equal loudness curve has been made. A-weighting is the unit typically used in government regulations and in situations involving people.

There are several other quantities that are quite useful in acoustics. For example:

- $L_{e q}$, the equivalent sound pressure level: this is the continuous, level corresponding to the average acoustical energy over a certain time. This unit is defined as:

$$
L_{e q}=20 \log \left(\frac{1}{T} \int_{t_{1}}^{t_{2}} \frac{p}{p_{o}} d t\right)
$$

$L_{e q}$ can be defined for any length of time, $\left(t_{2}-t_{1}\right)$ (e.g. 1 second, 1 hour, 1 day, 1 year, etc.);

- $L_{D N}$, a day-night equivalent sound level, similar to a 24 -hour $L_{e q}$ level, but weighting nighttime noise more heavily:

$$
L_{D N}=20 \log \left(\frac{1}{24}\left[\int_{7 a m}^{10 p m n} \frac{p}{p_{o}} d t+10 \int_{10 p m}^{7 a m} \frac{p}{p_{o}} d t\right]\right)
$$

- TL, transmission loss, which describes the decrease in pressure at a receiver location, as compared with the pressure $1 \mathrm{~m}$ from the source in a free field situation:

$$
T L=-20 \log \left(\frac{p_{\text {receiver }}}{p_{r=1 \mathrm{~m}}}\right)
$$

- EA, excess attenuation, which describes the sound pressure level at a receiver location, relative to what it would be in a free field situation:

$$
E A=S P L_{\text {refreefield }}=-20 \log \left(\frac{p(r)}{p_{\text {free }}(r)}\right) .
$$

Excess attenuation and transmission loss are quite similar quantities, differing only in the location of the reference value in the denominator of Equations (1.5) and (1.6);

- SEL, sound exposure level, which is the sum of the energy of a noise event from $t_{1}$ to $t_{2}$, compressed into 1 second:

$$
S E L=10 \log \left[\sum_{t_{2}}^{t_{1}} 10^{L_{e q} / 10}\right]
$$

SEL is often used to describe aircraft noise events;

- EPNL, the effective perceived noise level, in $\mathrm{dB}$, is a frequency weighted level that has a correction for the strong tonal components that tend to be perceived as more 
annoying than white noise (noise with equal energy at each frequency). It is defined as:

$$
E P N L=P N L_{\max }+10 \log \left(t_{10} / 20\right)+F(d B),
$$

where $\mathrm{PNL}_{\max }$ is the maximum perceived noise level, $\mathrm{t}_{10}$ is the duration, in seconds, where the perceived noise level is within $10 \mathrm{~dB}$ of the maximum level, and $\mathrm{F}$ is a correction for tonal components. This unit is common when measuring aircraft noise; and

- NEF, noise exposure forecast, is a decibel quantity used to predict community annoyance. It is defined as:

$$
N E F=E P N L+10 \log \left(N_{D}+16.7 N_{N}\right)-88(d B),
$$

in EPNdB, where $N_{D}$ is the number of daytime flights (07:00 - 22:00), and $N_{N}$ is the number of nighttime flights (22:00-7:00). This quantity is used primarily for landuse planning - NEF contours indicate which areas are suitable for different types of housing that will results in 'acceptable' noise levels for the residents. It is an averaged level, and is not effective at describing the type of noise events nor the annoyance that are problematic in this work.

These measures will be used to describe sound in this work; each of these can be measured in $\mathrm{dB}$ or $\mathrm{dBA}$, depending on whether the octave bands levels are unweighted or weighted.

Since it, would be tedious to use the A-weighting correction for each individual frequency, octave bands, which group frequencies around a center frequency, are often used to describe the sound spectrum, and to A-weight noise levels. Third-octave bands are also frequently used to describe the spectrum in finer detail. The band limits are defined according to

$$
\frac{f_{n+1}}{f_{n}}=2^{k},
$$

where on the left hand side, the numerator is the upper band cutoff, the denominator is the lower band cutoff, and $k$ defines the octave band width $(k=1$ for octave band, $k=1 / 3$ for third-octave band, etc.).

\subsubsection{Sources and Propagation}

Sound sources are often generalized into three ideal cases, each with their own type of geometrical spreading, resulting in a different amount of attenuation with distance. The 


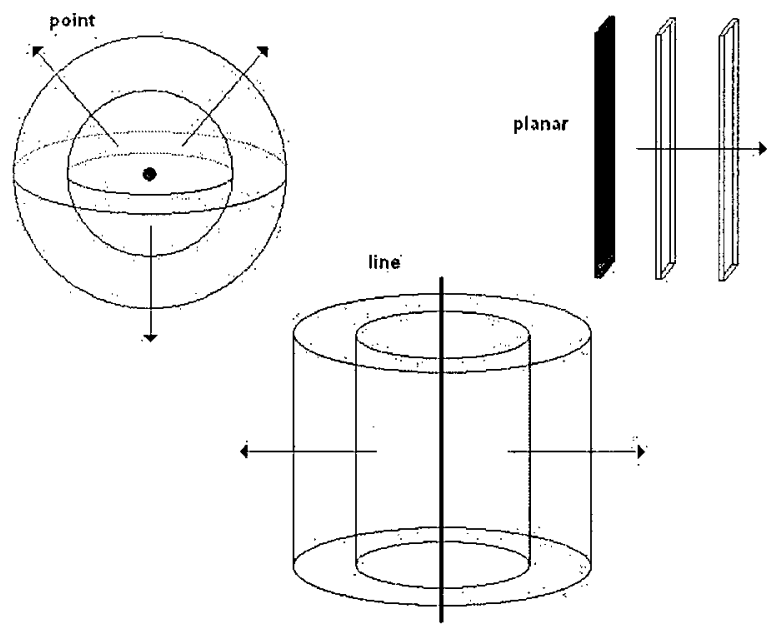

Figure 1.5: Geometrical spreading of a point, line, and planar source.

three cases are:

- point sources: where the geometrical spreading of wave fronts is spherical, resulting in attenuation of pressure $\propto \frac{1}{r^{2}}$ and of noise level of $6 \mathrm{~dB}$ per doubling of distance (i.e. aircraft flying high in the sky);

- line sources: where the geometrical spreading of wave fronts is cylindrical, resulting in attenuation of pressure $\propto \frac{1}{r}$, and of noise level of $3 \mathrm{~dB}$ per doubling of distance (i.e. roads with heavier traffic, and railway tracks with long trains); and

- plane sources: where the geometrical spreading of wave fronts is planar, and pressure and level do not vary with distance.

Far from the source, where the curvature resulting from a point source fades, the propagation can often be approximated as planar. These three types of propagation are illustrated in Figure 1.5.

The fluctuations of pressure, resulting in the transmission of sound, correspond to wave propagation through media. For a source and receiver, there are several types of waves that can exist $[6,19,22]$. These include:

- direct wave: sound that propagates along the direct line path from the source to the receiver; 
- reflected wave: the wave that reaches the receiver by reflecting from the ground. The amplitude and phase of this wave depend upon the ground impedance (a measure of resistance to the flow of pressure fluctuations of ground materials - see section 1.2.3);

- surface wave: this is a two dimensional wave that becomes trapped at the surface of grounds. For a surface wave to exist, the normalized admittance, $\beta$, (the inverse of impedance, were $\beta_{r}$ is the real, and $\beta_{x}$ is the imaginary component) must satisfy:

$$
\frac{1}{\beta_{x}^{2}}>\frac{1}{\beta_{r}^{2}}+1
$$

where $\beta=\beta_{r}-i \beta_{x}[19]$. For grounds with very large impedances (i.e. $Z \rightarrow \infty$ ), $\beta \rightarrow 0$ and Equation (1.11) can be approximated as:

$$
\beta_{x}>\beta_{r}
$$

This wave is a consequence of the theoretical derivation of the sound field, along with the direct and reflected waves, as will be shown in Chapter 3. The surface wave is unique in that it decays exponentially with height, and its amplitude decays slower than the direct and reflected waves $\left(p \propto r^{-0.5}\right.$ as opposed to $p \propto r^{-1}$, in cylindrical coordinates). The surface wave tends only to be noticeable close to the ground, and at larger distances $[6,22]$;

- ground wave: this is a (generally) low-frequency sound wave that propagates along finite impedance ground. A planar wave reflection coefficient is often used to approximate the reflected wave; this does not always predict levels as high as those measured in experiments (predominantly in experiments where both the source and receiver are placed very close to the ground). The explanation for this is that the wavefronts are spherical, and reflect according to the spherical reflection coefficient (the planar approximation does not quite hold): the ground wave is the part of the wave that hasn't been accounted for by the planar wave reflection coefficient:

$$
R_{p}+\left(1-R_{p}\right) F(w)
$$

where $R_{p}$ is the planar reflection coefficient, $r_{2}$ is the reflected wave (see Figure 1.6), and $F(w)$ is the boundary loss factor [19]. For a planar wave front, $F(w) \rightarrow 0$, and 
the ground wave disappears, leaving only the surface wave.

The interaction of these waves - particularly the former two - is essential to the determination of the overall sound level at the receiver. For example, over infinite impedance grounds, the difference in path length distance between the direct and reflected waves will create interference minima at odd integer half wavelengths, while the imaginary part of finite impedance grounds will cause a phase change, altering the interference pattern further. This phenomena is known as the ground effect: levels at receiver locations are higher or lower than expected if only the direct wave is considered. While many other types of acoustic waves exist (this list is not exhaustive), many of these attenuate quickly and need not be considered for long-range prediction.

\subsubsection{Ground Impedance}

The amplitude and phase of reflected waves can change upon incidence between two media; as a result, obtaining accurate values for the reflection coefficient is crucial for the creation of a reliable outdoor sound propagation model [19]. When dealing with a spherical source, the simpler plane-wave reflection coefficient can be used to approximate the spherical-wave reflection coefficient when the source and receiver are farther apart from one another than their heights above the ground. The planar-wave reflection coefficient is defined as:

$$
R=\frac{Z_{2} \sin (\phi)-Z_{1}}{Z_{2} \sin (\phi)+Z_{1}}
$$

as illustrated in Figure 1.6, the subscripts refer to medium 1 (air) and medium 2 (ground), $Z$ is the impedance, and $\phi$ is the angle shown in Figure $1.6[6,23]$. It is apparent from Equation (1.14) that the impedance, $Z$, is of utmost interest in determining how waves reflect. A discussion on existing methods for calculating ground impedance, techniques for actual measurements, and the sensitivity of overall sound pressure levels with small variations in the ground impedance follows.

The acoustics impedance of a medium (a complex quantity), is the ratio of pressure to normal particle velocity of the material:

$$
Z=\frac{p}{v}
$$




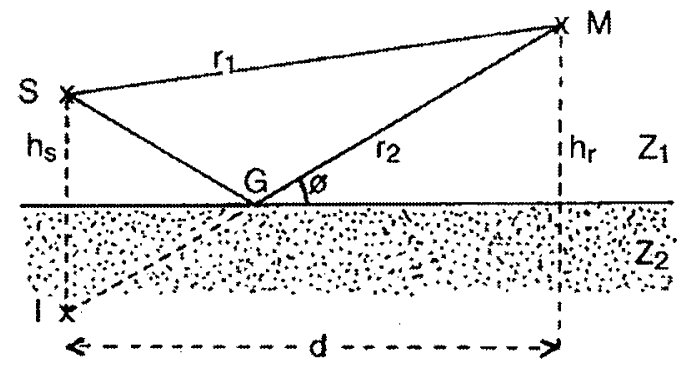

Figure 1.6: Reflection of a sound wave upon incidence [6]

The normalized (specific) acoustic impedance is more commonly used; it is the acoustic impedance divided by the impedance of air. This is a dimensionless quantity $\left(Z=\frac{p}{v} /(\rho c)\right)$. The impedance of a medium depends mostly on the flow resistivity, $\sigma$, a measure of the resistance to the flow of air, and is usually measured in mks rayls $/ \mathrm{m}$, or $\mathrm{Pa} \mathrm{s} / \mathrm{m}^{2}$. The impedance of a medium also depends on porosity. The porosity of a material, determined by the size, shape, and distribution of air-filled pores, influences how much energy is converted to friction and thermal losses upon incidence $[6,19]$.

Impenetrable, hard surfaces have infinite impedances; sound waves incident on surfaces of infinite impedances are completely reflected (no sound transmission, no energy loss from the pressure wave). Much more common, however, are finite impedance surfaces: grass, for instance, has an flow resistivity of $\sim 200 \mathrm{kPa} \cdot \mathrm{s} \cdot \mathrm{m}^{-2}$ which corresponds to a normalized ground impedance of $\sim 11.5+11.5 i$ and a reflection coefficient of $R \sim 0.88$ at 1000 $\mathrm{Hz}$. The complex nature of impedance is the result of the complex pressure and particle velocity. The real (or resistive) part of impedance relates how much energy is lost (i.e. attenuation), while the imaginary (or reactive) part, sometimes referred to as the compliance or springiness of a material, determines the phase change upon reflection. The frequency dependence of a medium is important: for longer wavelengths, a medium may appear to have infinite impedance, while at shorter wavelengths, the same medium can appear to have finite impedance.

Another important characteristic of ground surfaces is whether they are locally reacting or extended reacting. A material is locally reacting if its impedance is the same for normal and oblique incidence waves. An extended reacting medium is one whose impedance is a function of incidence angle, and whose pressure and normal velocity component at the surface will be influenced by the pressure and velocity nearby. Surfaces are often assumed 
to be locally reacting $[2,16]$. For locally reacting surfaces, a point image source in the lower half-plane can be used for modeling, as illustrated in Figure 1.6. For extended reacting surfaces, however, no single image point exists from which the reflected wave can be assumed to radiate: a 'fuzzy' point, (i.e. a flattened and stretched point) is required. The impedance of many ground surfaces (i.e. grass [6]) does not depend strongly on the incident angle.

\section{Experimental approach to ground impedance measurement}

There are several ways by which normal acoustic impedance can be measured. For instance, the ground can be irradiated by sound from loudspeakers, and the pressure and volume velocity at the surface measured [24]. A more common method is to measure the interference pattern of the direct and reflected waves, using an impedance tube or in the free field, and then find the normalized impedance by calculating the reflection coefficient $[22,25]$. While certain methods have been found to be particularly useful in specific cases (i.e. low frequencies, grazing angles, materials in situ, etc.), the setup for measurement can be fastidious. Consequentially, the commercially available impedance tube has become a popular method.

There are several limitations to impedance tubes. For instance:

- measurements can only take place at normal incidence - this is problematic for extended reacting surfaces;

- the size of the tube restricts the frequencies at which the impedance can be measured: the longer the wavelength, the larger the tube diameter must be; and

- obtaining samples can be difficult, particularly when trying to measure a material in situ.

\section{Theoretical approach to ground impedance}

Delany and Bazley developed empirical equations to predict the real and imaginary components of the impedance of fibrous and porous materials, which are still often used today [26]. Their power-law equations are a function of frequency and flow resistance. For the real $(\mathrm{R})$ and complex $(\mathrm{X})$ parts $\left(Z_{o}=R+j X\right)$, Delany and Bazley found:

$$
\frac{R}{\rho_{o} c_{o}}=\left(1+9.08\left(\frac{f}{\sigma}\right)^{-0.75}\right) \text { and } \frac{X}{\rho_{o} c_{o}}=\left(-11.9\left(\frac{f}{\sigma}\right)^{-0.73}\right)
$$


where $f / \sigma$ is measured in $\mathrm{cm}^{3} / \mathrm{g}$. This equation can be rewritten in terms of effective flow resistivity, $\sigma_{e}$ as [19]:

$$
Z==1+0.0571\left(\frac{f}{\sigma_{e}}\right)^{-0.754}+i 0.057\left(\frac{f}{\sigma_{e}}\right)^{-0.732}
$$

The flow resistance of the materials measured in Delany and Bazley's work ranged from 2 to 80 CGS rayl (1 CGS rayl $=10 \mathrm{mks}$ rayl) and the frequencies ranged from 250 to 4000 $\mathrm{Hz}$. These measurements were for plane-waves and normal incidence (assumptions often made in modeling). In 1983, Embleton, Piercy and Daigle at the NRC in Ottawa further extended this body of knowledge with their publication on flow resistivities for common ground surfaces found both experimentally and theoretically [27].

More recently, Attenborough has worked extensively developing impedance models. Models published throughout the 1980's and 1990's have involved anywhere from 1 to 5 parameters. While Attenborough found flow resistivity to infiuence impedance the most, the ground structure was also found to play a significant role. In addition to flow resistivity, Attenborough's models include $[19,28,29]$ :

- porosity $(\Omega)$, which has to do with the air-filled pores, resulting from how densely packed the material is;

- tortuosity $(q)$, which has to do with the 'twistiness' of the pores;

- pore shape factor ratio $\left(s_{f}\right)$; and

- grain shape factor $(g)$.

Tortuosity is related to the porosity and grain shape factor as $q^{2} \equiv \Omega^{-q}$.

The four-parameter model, which is frequently used in predictions $[2,15]$ is:

$$
Z=\left(\frac{4 q^{2}}{3 \Omega}+i \frac{s_{f}^{2} \sigma}{\rho \omega}\right) \frac{w / c}{k}
$$

in which the wave number $k$ equals:

$$
k=\frac{w}{c} \sqrt{\gamma \Omega} \sqrt{\left(\frac{4}{3}-\frac{\gamma-1}{\gamma} N_{p r}\right) \frac{q^{2}}{\Omega}+i \frac{s_{f}^{2} \sigma}{\rho \omega}}
$$

While the more sophisticated models with more input parameters can theoretically predict impedances with better accuracy, poorer predictions are obtained if the input parameters are not chosen correctly. In many cases, models with fewer parameters, such as 


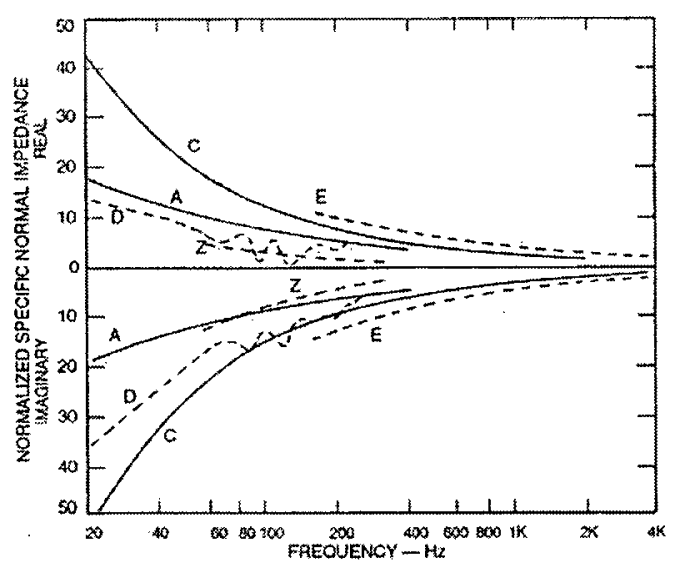

Figure 1.7: Normalized specific acoustic impedance: Measured ( - - ): curve E by Embleton et al.; curve $D$ by Daigle and Stinson; and curve $Z$ by Zuckerwar; and theory (-): curve $A$ by Attenborough (5-parameter model); and curve C by Chessell (extension to Delany and Bazley's work) [6].

Attenborough's two-parameter model, are a better choice, particularly for high flow resistivities and locally reacting grounds [29]. The two-parameter model is expressed as:

$$
Z=\frac{\sqrt{\frac{\sigma_{e}}{f}}}{\sqrt{\pi \gamma \rho}}(1+i)+\left(\frac{i c \alpha}{4 \gamma \omega \Omega}\right)
$$

where $\gamma$ is the ratio of specific heat for air, $\sigma_{e}$ is the effective flow resistivity $\left(\sigma_{e}=4 s_{p}^{2} \sigma / \Omega\right)$, $\alpha=\frac{4}{3}-[(\gamma-1) / \gamma] N_{p r}$, where $N_{p r}$ is the Prandtl number, $\omega=2 \pi f$, and $\rho$ is density (of air). The resistive and reactive parts of impedance calculated by different models have been plotted in Figure 1.7 for comparison.

\subsubsection{Atmospheric Absorption}

Sound waves lose energy as they propagate in a medium such as air. This occurs because of conduction, viscous, and molecular absorption, resulting from the induced vibrations of molecules in air (particularly oxygen-water molecules at higher frequencies, and nitrogenwater molecules at lower frequencies) [6]. Pressure, temperature and humidity all affect air absorption in a complicated, frequency-dependent way. In general, at constant temperature, an increase in relative humidity will decrease air absorption. At constant relative humidity, an increase in temperature will decrease air absorption for high and low frequencies, and 
increase air absorption for mid-frequencies [30].

Air absorption is often neglected in the calculation of sound levels, particularly in situations with low frequencies and shorter distances, for which the absorption will be very small. Outdoors, however, when dealing with distances of several hundred meters to kilometers, air absorption can have a major effect on sound - particularly high frequencies - and cannot be ignored.

Another potential source of sound attenuation is vegetation. Vegetation - be that trees, plants, or bushes - is commonly planted for noise control purposes. In general, this can be very pleasing aesthetically; however a single row of trees does not significantly alter sound (a wide, dense band is required before a change is noticeable [19]). Some work has been done modeling trees as cylinders (large and small, for trunks and branches), such to produce a new propagation constant[22]; Price et al. used a similar method to identify three types of attenuation: via the ground effect (a forest floor has a more absorbent layer), scattering by the trunks and branches, and viscouls friction from the leaves $[19,31]$. Other models have reported predictions of $5 \mathrm{~dB}$ of attenuation resulting from a coniferous forest $100 \mathrm{~m}$ wide $[19,32]$.

\subsubsection{Inhomogeneous Atmosphere}

In a homogeneous atmosphere (non-refracting, i.e. constant temperature, no wind), sound will propagate following the wavefronts described in Section 1.2.2. The atmosphere is, however, inhomogeneous: humidity, wind, and temperature lapses and inversions create non-uniform vertical sound speed profiles, resulting in the refraction of the wavefronts. The creation of quiet areas, known as shadow zones, and areas where the sound is focused along a 'tunnel,' known as ducting, are a direct consequence of refraction. These effects are illustrated in Figure 1.8.

Temperature lapses, where the temperature decreases with height, are most common during the day time. This is when solar radiation heats the ground more quickly than the air above it, resulting in a negative temperature gradient $[2,19]$. Temperature inversions, on the other hand, typically occur at night once the ground has cooled off more quickly than the atmosphere above it, resulting in a positive temperature gradient $[6,19]$. 


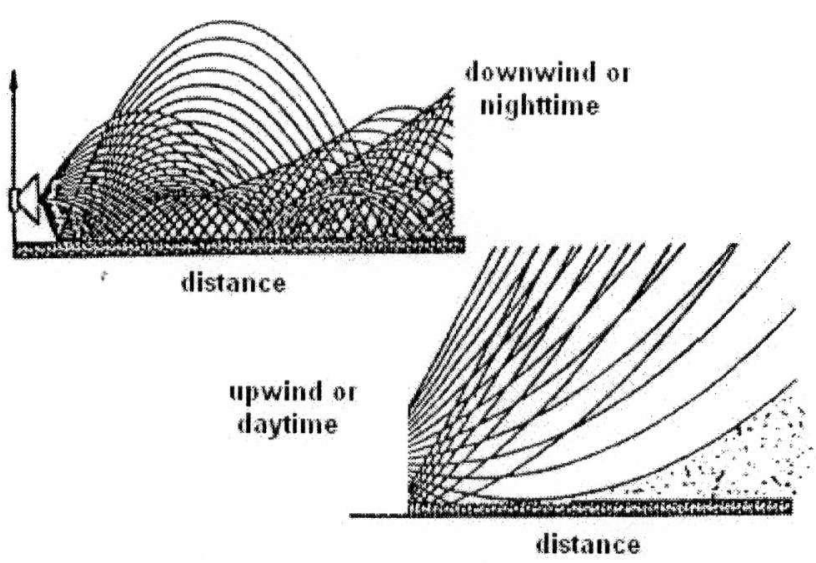

Figure 1.8: Wave refraction in downwind (similar to temperature inversion conditions) and in upwind (similar to temperature lapse conditions) [7].

\subsubsection{Turbulence}

Thus far, it has been assumed that wind and temperature profiles are constant with time - in reality, this is incorrect. Atmospheric turbulence, which results in variations in the mean velocity of sound, is created primarily by random wind fluctuations, resulting in the scattering of sound as it propagates $[2,19,33]$. The effects of turbulence are particularly noticeable at large source-receiver distances and during upward refracting conditions, where in shadow zones sound pressure levels can be up to $\sim 30 \mathrm{~dB}$ higher than expected in a turbulence-free situation $[2,16]$. For long-range predictions during upwind and temperature lapse conditions, including turbulence while modeling sound propagation can reduce errors in the order of tens of decibels $[9,34]$.

From basic fluid dynamics it is known that turbulent flows are those whose Reynolds numbers are above a critical value. The Reynolds number is a dimensionless parameter that relates the inertial (momentum) forces to viscous forces. It is related to the flow rate, a physical dimension (e.g. the height above the ground at which there is flow), and the viscosity of the fluid. The Reynolds number for air flow above the ground is defined as [2]:

$$
N_{R}=v L / \nu
$$

where $v$ is the velocity, $L$ is the height above the ground through which the flow exists, and $\nu$ is the viscosity. One reference reported the critical Reynolds number along a street in New York city to be $\sim 4000-5000$ [35]. A typical value for $\nu$ is $0.15 \mathrm{~cm}^{2} / \mathrm{s}$ [2]. This 
suggests that, at a height of $2 \mathrm{~m}$, the minimum wind velocity required to create turbulent flow is approximately $0.04 \mathrm{~m} / \mathrm{s}$ (this is a very weak wind, and the value will decrease with increasing height). While the values used in these calculations are estimates, this weak wind velocity for turbulent flow suggests that turbulence exists in the atmosphere in most situations (rarely is the wind speed less than $0.04 \mathrm{~m} / \mathrm{s}$ ). While Reynolds number is fundamental when choosing how to model flows, for atmospheric acoustics, turbulence can always be assumed to be present, causing fluctuations in wind speed and temperature, resulting in the scattering of sound $[2]$.

Although turbulence is created by 'random fluctuations' in wind speed, the structure of turbulence in the atmosphere is not completely arbitrary. As described by Salomons in [2], air is a viscous fluid. Boundary layer conditions, such as friction along the ground, create a shear stress in a volume element of air, resulting in deformation of the volume element, creating eddies. Larger eddies on the order of the height of the flow above the ground are formed first; medium and then smaller eddies are subsequently induced when wind shears off the larger eddies. Once the eddies reach a critical size $(\sim 1 \mathrm{~mm})$, their energy is dissipated into viscous and thermal energy [2].

In reality, turbulence scatters sound in a frequency-dependent manner [36]. The size of eddies affects wavelengths of the same size [2]. It has been reported that it is the highest frequencies that are most affected by turbulence [16], suggesting that smaller eddies are more prominent than larger eddies. When modeled, however, the frequency dependence is neglected [36].

Turbulence can be categorized as 'strong,' 'weak,' or somewhere in between. These classifications are defined by the relative size of intensity fluctuations to the mean intensity: for weak turbulence, the fluctuations are less than $\sim 10 \%$ of the mean; for strong turbulence, the fluctuation size is close to the mean $[19,37]$. To date, only weak turbulence has been modeled successfully in atmospheric acoustics [34]. That said, particularly in strong upward refracting atmospheres and at larger distance, changes in destructive interference under weak turbulence conditions for outdoor sound propagation can be quite large [8].

Variations in sound speed due to turbulence can be incorporated using the acoustic refractive index, $n$. This quantity is the ratio of a constant sound speed (e.g. at ground level) to the actual speed of sound. It is defined as [2]: 


$$
n=c_{o} / c_{\text {eff }}+\mu
$$

where $c_{o}$ is a constant sound speed, and $c_{\text {eff }}$ is the effective sound speed. The fluctuations in the index of refraction, $n$, are represented by $\mu$ which, when averaged over time, equals zero $(\bar{\mu}=0)$.

If the temperature fluctuation, $T_{t}$, and wind velocity fluctuation, $u_{t}$, are known, the fluctuations in the index of refraction can be calculated according to [2]:

$$
\mu=-\frac{T_{t}}{2 T_{0}}-\frac{u_{t}}{c_{0}}
$$

These fluctuations can be difficult to obtain. If details on the mean and standard deviations are known (i.e. from measurable meteorological data, the mean and standard deviation values of temperature and wind fluctuations are measured), the fluctuation in the index of refraction can be calculated for a precise moment from Equation (1.23), or for an average, as shown in Equation (1.24). The following expression for the fluctuations in the index of refraction has been derived assuming the fluctuations in wind and temperature are normally distributed $[8]$ :

$$
\left\langle\mu^{2}\right\rangle=\left(\frac{\sigma_{\nu} \cos (\theta)}{c_{o}}\right)^{2}+\frac{\sigma_{\nu} \sigma_{T} R \cos (\theta)}{c_{o} T_{o}}+\left(\frac{1}{2} \frac{\sigma_{T}}{T_{o}}\right)
$$

where $\sigma_{\nu}$ and $\sigma_{T}$ are the standard deviations of wind and temperature, $\theta$ is the angle between the average wind direction and the direction of sound, $R$ is the cross correlation between wind and temperature fluctuations, and $c_{o}$ and $T_{o}$ are the average sound speed and temperature [8]. More recent studies have looked into the relative contributions of wind and temperature fluctuations, and found that wind effects are usually greater than temperature effects [34]. Figure 1.9 shows typical fluctuations of wind and temperature with time and frequency. The fluctuations can also be derived from theory, as will be discussed in following sections.

\section{Physical Effects of Turbulence on Sound Level and Spectrum}

Turbulence can be approximated by applying additional phase and amplitude fluctuations to sound pressure levels. In effect, this results in wavefronts, originally in phase and of the same amplitude, becoming out of phase and of different magnitude $[6,8,34]$. 

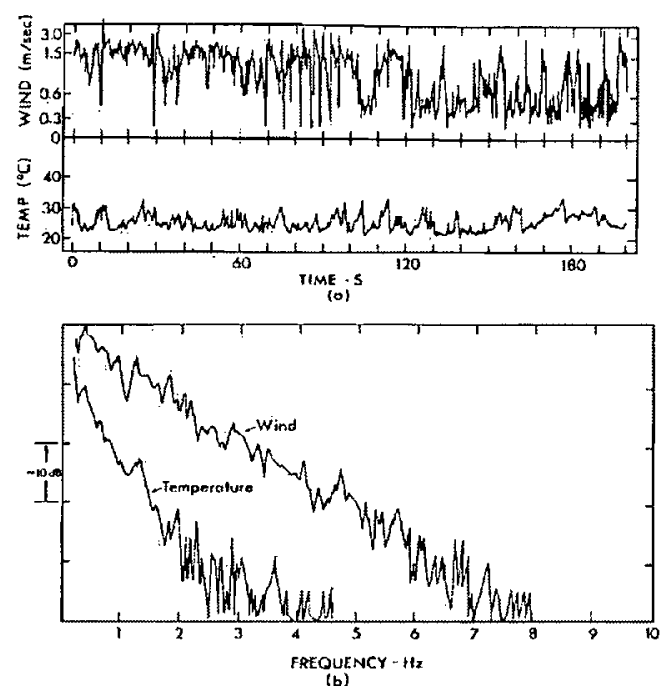

Figure 1.9: Turbulence record of wind and temperature fluctuations (a) with time, and (b) with frequency [8].

In a non-refracting (homogeneous - i.e. no wind, and temperature is constant with height) atmosphere, the addition of turbulence reduces the depth of the minima expected from the interference of the direct and reflected sound waves. This dip will also be spread across a wider frequency range. This is most noticeable with sources and receivers close to the ground and at short source-receiver distances: for the positions/frequencies where sharp minima once existed, the sound pressure level will not be as low. In a downward refracting atmosphere (i.e. temperature inversion or downwind), the effects of turbulence are similar to those in a non-refracting atmosphere: the depths of the interference minima will be smaller, but spread over a larger range $[2,9]$.

A different effect, however, is seen with an upward refracting atmosphere (i.e. temperature lapse, and/or sound propagation in the upwind direction). In this case, sound will be scattered into the shadow zone, actually increasing the sound pressure level in that region. Sound pressure levels in shadow zones are typically $20-30 \mathrm{~dB}$ lower than if there were no shadow zone $[2,9]$. However, with turbulence, shadow zones are $\sim 10 \mathrm{~dB}$ higher. This situation is of particular importance over longer distances, where the receiver is in the shadow zone [33]. This result has been confirmed experimentally [33].

Gilbert et al. describe the upward refracting case by dividing it into three regions, as illustrated in Figure 1.10 [9]. In the first region, where spherical (geometrical) spreading 


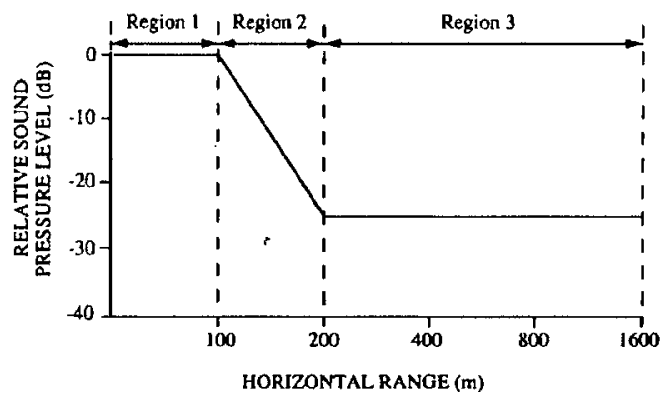

Figure 1.10: Relative sound-pressure level as a function of range in an upward-refracting atmosphere. Values here are typical of a strong upward refracting atmosphere [9].

dominates, there is no attenuation in the relative sound pressure level. In Region 2, which corresponds to the edge of the shadow zone, there is a sharp drop in the relative sound pressure level. This drop levels off at a constant value once inside the shadow zone (Region 3), usually 20-30 dB below the level in Region 1 [9].

\section{Numerical Representation of Turbulence}

There are two ways to 'generate' turbulence in sound propagation models: using experimental data, as discussed earlier, and using numerical representations, such as phase screens [38]. Salomons [2] describes how the correlation and structure functions are used, depending on the homogeneity of the turbulence, to produce random fields characterizing turbulence. These functions represent the fluctuations of the temperature and wind from the average levels. If the turbulence is homogeneous (i.e. independent of position), the correlation function, $B$, is used; if the turbulence is inhomogeneous, the structure function, $D$, is used. Both the correlation function and the structure function can be isotropic (where the difference is a scalar quantity) or non-isotropic (where the difference is a vector). The correlation function is only reliable at describing turbulence for short ranges and weak turbulence $[2,33]$.

Salomons [2] also shows how the correlation function can be generated indirectly by taking the Fourier transform of a spectral density. Spectral densities have been characterized by several spectrums - the Gaussian, Kolmogorov, and vón Kármán being the most common in atmospheric acoustics $[2,16]$. None of these schemes is ideal, as they are for a homogenous, isotropic atmosphere: in real world situations, turbulence increases with height, and the wind direction is a factor (in other words, turbulence should be modeled as 
inhomogeneous and non-isotropic) [2]. The two-dimensional spectral density function for the Kolmogorov spectrum, $F(k)$, is:

$$
F(k)=C^{2} \frac{\Gamma^{2}\left(\frac{1}{2} p+1\right) 2^{p}}{2 \pi^{2}} \sin \left(\frac{1}{2} \pi p\right)|k|^{-p-2}
$$

where $p=2 / 3, \Gamma$ is the gamma function, and $k$ is the wave number. The two-dimensional spectral density function for the von Kármán spectrum is:

$$
F(k)=\mu_{o} \frac{\Gamma(8 / 6)}{\Gamma(1 / 3) \pi} \frac{a^{2}}{\left(1+k^{2} a^{2}\right)^{8 / 6}}
$$

where $\mu_{o}$ is the standard deviation of $\mu$ and represents the intensity of the turbulence, and $a$ is the correlation length. More complicated than the Gaussian spectrum, these spectrums are also considered to be more realistic.

\section{How turbulence applies to modeling}

Temporal changes in sound pressure levels caused by atmospheric turbulence are generally on the order of a few seconds to minutes [2], however changes in temperature of up to $5^{\circ} \mathrm{C}$ in a few seconds are not unusual [6]. While a short single sound pressure level measurement will capture the effect at a particular instance, it does not represent the variability with time. As a result, measurements that include the variability of turbulence should be averaged over an extended period of time (i.e. several minutes).

A 'snapshot in time' is often used when incorporating turbulence in computer models: here it is assumed that the speed of sound is much faster than any atmospheric variations (so that, at that given moment, no atmospheric changes occur) [2]. This is equivalent to one short sound pressure level measurement (no averaging). For measurements taken over a longer period of time, multiple 'snapshots in time' are predicted, and then logarithmically average, to account for the turbulent variations with time. This method is called the 'Frozen Medium Approach' [2]. Turbulence is concerned with levels over intervals of a few seconds to minutes. To compensate for its dynamic nature, when modeling turbulence, several runs are usually averaged. 


\subsection{Annoyance}

Annoyance is a complex, subjective issue. Assessing it scientifically, with a measure that will express both the quantity and quality of annoyance, is extremely challenging. To add to the complication, annoyance thresholds (for example, the minimum sound level at which an individual will become annoyed) vary from person-to-person, day-to-day and, depending on what the individual is doing, on their mood, and on previous experience. One objective of this research project was to predict noise levels in communities so that run-up options that minimize annoyance could be selected; thus, some kind of quantification method relating annoyance to noise levels was required. There has been a great deal of work done on annoyance from overhead flights (e.g. relating the number of events above a certain noise level to the number of people annoyed). No information was found which specifically related run-up noise to anmoyance. Because the run-ups in question take place during the night, sleep disturbance was chosen as the measure to gauge annoyance.

\subsubsection{Annoyance and Sleep Disturbance}

Sleep disturbance is a broad subject and can be assessed in many ways. Some of the more common dependent variables include the number of awakenings, the change in sleep stage (i.e. the transition to a lighter stage), and 'motility' (small movements) in a pre-determined period of time. There are a number of independent variables, often A-weighted, that can be predicted from the dependent variables, such as the sound exposure level (SEL), the maximum level $\left(L_{\max }\right)$, and the day-night equivalent level $\left(L_{D N}\right)$. Models based on events (i.e. SEL) are generally preferred to those that are continuous (i.e. daily averages), since one single event can be enough to cause sleep disturbance. Other annoyance issues arising from noise include:

- psycho-social variables (how a person feels about aircraft - if they are afraid of airplanes crashing nearby, how passionate they are about aviation, etc.) $[39,40,41,42]$; and

- health risks, such as heart disease, mortality, mental instability, depressiveness [43], low birth rates [44], memory loss (long-term), reading and concentration, and stress responses (i.e. systolic blood pressure, high levels of resting adrenaline and nora- 


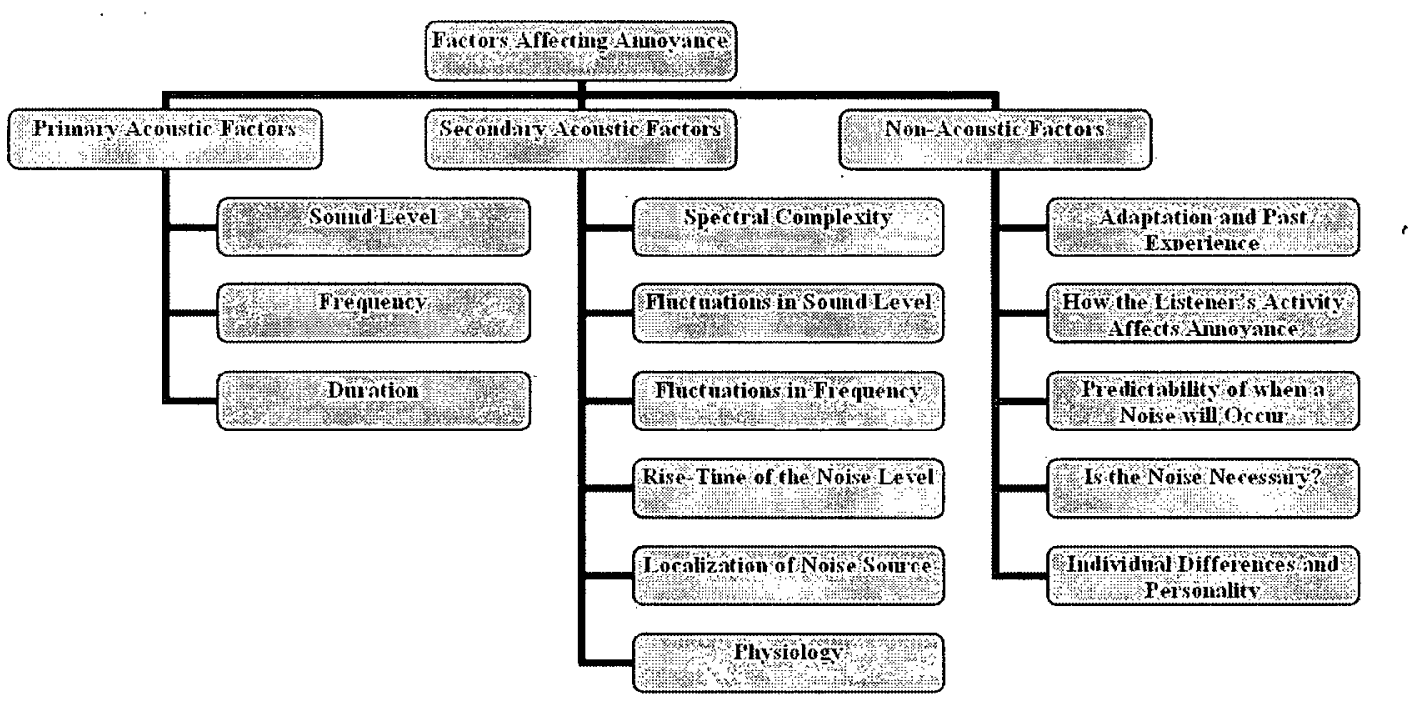

Figure 1.11: Factors affecting annoyance according to FICAN [10]

drenaline) [45].

FICAN, the Federal Interagency Committee on Aviation Noise has listed factors affecting annoyance, which have been reproduced in Figure 1.11. This shows how complicated annoyance is - each box in Figure 1.11 is a topic in itself [10].

\subsubsection{Quantification}

There are two environments for determining the relationships between noise and sleep disturbance: in a laboratory setting, and in the field (i.e. in people's homes). Experiments have also been done to combine the two (i.e. by making noise in people's homes). Many researchers have found that the results between the two environments vary significantly, with a much larger percentage of people annoyed/awakened in a laboratory than in the field [46]. This may in part have something to do with habituation (people adjust to noise disturbances fairly quickly). It is even suggested by some that the difference between field and laboratory studies is so large that results from one setting should not be transferred to the other [46]. While a higher percentage of people are disturbed by noise in a lab than in the field, data obtained in the field has been found to be more reliable in real-life applications [46]. Since neithèr type of study was conducted as part of the current research project, existing information and relationships will be used to gauge annoyance. 
All information found relating noise levels to annoyance is based on the noise level inside .the home, close to the person sleeping; unfortunately only outdoor levels are predicted in this work. The difficulty in predicting noise levels inside a house is that it requires predicting the transmission loss through walls into homes, for which there is a vast amount of variability from house to house. There is currently no standard for sound-proofing houses in the communities around YVR, and each home has its own geometry and orientation.

An earlier study used a transmission loss of 25. dBA to compare outdoor and indoor $L_{\text {max }}$ levels [47]. Bradley [48] considered the situation more thoroughly, and developed a program, Insulating Buildings Against Noise from Aircraft (IBANA-Calc), to predict indoor sound levels from outdoor sound levels in Vancouver and Toronto. To use IBANA-Calc in combination with the current sound propagation model being developed for annoyance predictions would not only add unnecessary complications, it would be next to impossible, since for IBANA-Calc, the noise reduction is measured in each room individually, and the dimensions and absorption of each must be known. Additionally, the coincidence effect, where at a certain frequency there is an increase in transmission through a wall as it acts more like a resonator than a barrier, will vary unpredictably from house to house, creating unexpected higher sound levels indoors at certain frequencies. The assumption that one value would be good for any house would likely lead to a decrease in the accuracy for the prediction of the percentage of annoyed people during a noise event.

An accurate representation of community reaction can be useful in understanding the effect of noise in communities. Residents and airport workers alike can contact YVRAA by phone, email, fax, in person, etc., to submit noise complaints. Complaints corresponding to times of engine run-up tests suggest that those run-ups were loud enough to disturb members of the community. While this is one way to identify problematic run-ups, complaints are not the most reliable and appropriate indicator [49]. For instance, when someone is annoyed, they won't necessarily bother to call in a complaint (based on their tolerance, health, cultural attitude, socio-economic factors, etc.), or even know how to do so (although many airports worldwide have a wide range of ways to do so). This lack of consistent and reliable community response makes it more difficult to determine the true percentage of the population that is annoyed in various circumstances.

A study done at the Dar es Salaam International Airport in Tanzania, studying noise levels and annoyance/complaints, for instance, concluded that, while people often said they 
were annoyed by the noise (some settlements were just over $200 \mathrm{~m}$ from the runway) and experienced maximum noise levels of $86 \mathrm{dBA}$, not one person surveyed said they had ever submitted a complaint [50]. In Spain, a survey that sampled 1800 people living near airports found $64 \%$ of people had never complained and, of those who did, $29 \%$ of the complaints were directed to relatives or neighbours rather than to the people responsible for the noise ( $15 \%$ of the sample population was very much annoyed, $23 \%$ rather annoyed, and $24 \%$ moderately annoyed) [49]. Consequently, the community reaction should be considered; however it must be remembered that complaints do not provide a true gauge of the community's annoyance.

\subsubsection{Relationships}

Since no relationship was found that directly relates outdoor sound levels to sleep disturbance, a compromise was made. Outdoor sound exposure levels were chosen to represent, the indoor levels (assuming the worst case scenario, when all windows are open), and three relationships relating the percentage of people awakened were considered.

In 1992, FICON, the Federal Interagency Committee on Noise, and in 1997, FICAN, the Federal Interagency Committee on Aviation Noise, developed dose-response relationships between indoor SEL levels and the percentage of awakenings. FICON's dose-response was based on the percentage of people expected to be awakened, while FICAN's dose-response was based on the maximum percentage of people expected to be awakened. The relationships are:

$$
\begin{aligned}
& \text { FICON: \% Expected Awakenings }=0.000007079 S E L^{3.496} \\
& \text { FICAN: \% Maximum Awakenings }=0.0087(S E L-30)^{1.79}
\end{aligned}
$$

FICON's relationship was found using both laboratory and field data, and was thought to overestimate the percentage of awakenings, while FICAN's relationship was found using field data only. These two relationships, along with the field studies used by FICAN, are shown in Figure 1.12 [11].

In 2001, American National Standards Institute (ANSI) published a standard that relates the indoor sound exposure level to the percentage of people awakened in the field and laboratory [12]. The expression for field results is: 


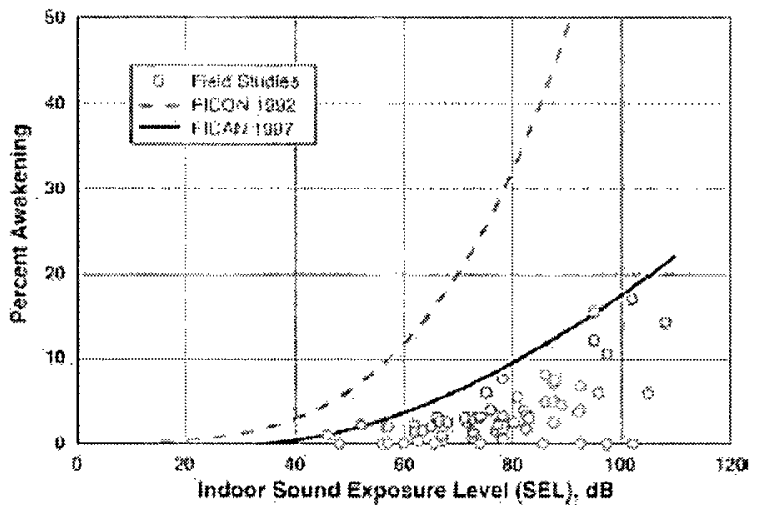

Figure 1.12: Comparison of FICAN and FICON sleep awakening relationships [11].

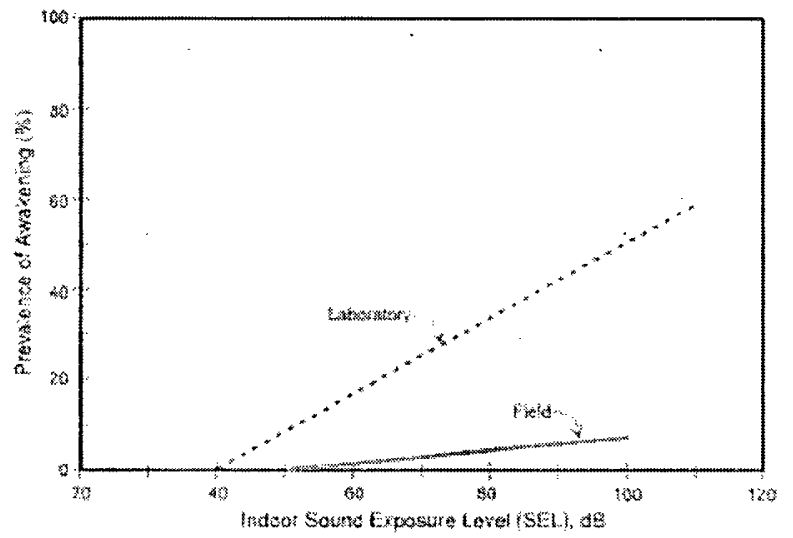

Figure 1.13: Prevalence of awakening in laboratory and field environments based on indoor sound exposure level [12].

$$
\% \text { Awakenings }=-7.02+0.14 \mathrm{SEL}
$$

Figure 1.13 shows the difference between laboratory and field measurements [12].

These equations should be used with caution: annoyance depends on many factors and relationships do not necessarily transfer from one community to another. 


\subsection{Research Objectives}

Given the theory described in the previous sections, the research objectives for this work are as follows:

- to characterize jet aircraft run-up noise through measurement;

- to modify an existing outdoor sound propagation model, the Green's Function Parabolic Equation, or GFPE, to better represent effects the ground and atmosphere have on sound propagation, and also to obtain more informative output values;

- to evaluate the model with measurements and predictions in literature, to ensure all modifications result in accurate approximations; and

- to use the modified model to predict run-up noise and annoyance predictions.

This thesis project describes how these objectives were achieved.

\subsection{Thesis Outline}

The ideas discussed in this chapter will be investigated further in the following chapters, to realize the objective of predicting noise levels and annoyance in communities resulting from aircraft engine run-up noise.

In Chapter 2, the procedures and results for the measurement of sound pressure levels and radiation characteristics of a small jet aircraft during a run-up, will be discussed. The body of knowledge relating to engine run-up noise is very small; these results compliment earlier measurements made on propeller aircraft $[13,18]$.

In Chapter 3, theory concerning the sound propagation model used for this thesis work, the GFPE model, will be presented. Modifications to the GFPE code to achieve the objectives of the current work are also discussed.

The modifications made to the GFPE model in Chapter 3 are evaluated in Chapter 4, by comparing prediction results with measured and predicted results from the literature. The model is then used in Chapter 5 to predict sound pressure levels corresponding to run-up events that were thought to have been the cause of noise complaints in nearby communities.

Results are summarized and the objectives assessed in the final chapter, Conclusions. 


\section{Chapter 2}

\section{Run-up Noise Measurements}

\subsection{Introduction}

Subsequent to aircraft maintenance, aircraft operators must perform an engine run-up before the aircraft can return to service. Aircraft operators are expected to seek permission for engine run-ups prior to the event; the run-ups should only take place once YVRAA Airport Operations gives the aircraft operator approval. The aircraft operator must abide by the approved location, heading, time slot, duration, and engine level, given by YVRAA Airport Operations.

In order to predict sound levels in communities, the source levels from aircraft radiated in the direction of the receiver of interest, are required as a model input parameter. No current information on the directivity, spectrum, or noise level of aircraft doing engine run-ups was found in the literature [51], other than from the work on propeller aircraft engine run-ups done previously by UBC and YVRAA $[18,20]$. Since approximately $50 \%$ of aircraft activities at YVR are for jet aircraft, which have very different sound-radiation radiation characteristics than propeller aircraft, arrangements were made with the Air Canada Jazz airline operating at YVR to take measurements of a small commuter jet aircraft, the Canadair CRJ200.

Noise measurements of a run-up for a CRJ200 (see Figure 2.1), provided by Air Canada Jazz, were made on Thursday, August 11, 2005, in conjunction with a taxiing exercise. The purpose of these measurements was to determine the energetic and directional radiation characteristics (spectra, directivities) of a jet aircraft that flies at YVR, and to determine how it is similar or different to those for a propeller aircraft. The CRJ200 is smaller than many jet aircraft; its noise radiation doesn't necessarily represent that of other jet aircraft. Noise levels were measured at two heights, at 15 locations around the aircraft, $40 \mathrm{~m}$ from the centre of the source, and at three power settings. This was done by recording the sound signals detected by sound level meters onto DAT tapes (digital audio tapes); the tapes were 


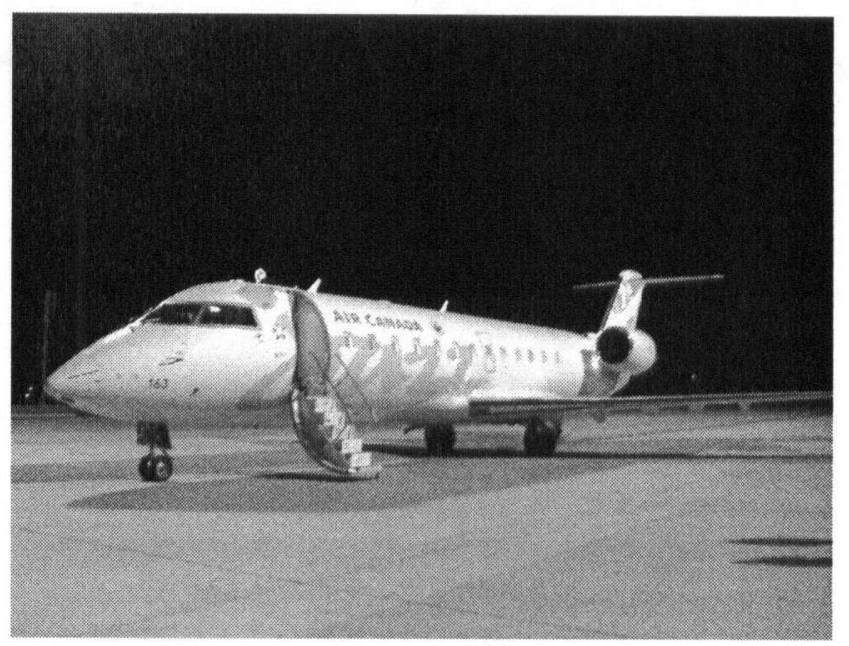

Figure 2.1: The CRJ200 aircraft.

subsequently analyzed on computer. This chapter describes the process involved, and the resulting levels and directivities of the CRJ200.

The CRJ200 is a regional jet aircraft, designed by Bombardier to carry 50 passengers. It has two General Electric CF34-3A1 turbofan engines, located mid-way between the wings and the tail of the aircraft. Its overall length is $26.77 \mathrm{~m}$, overall height is $6.22 \mathrm{~m}$, and its wing span is $21.3 \mathrm{~m}[52]$. The exit plane of the engines (where the exhaust exits the engines, and the location of the aircraft assumed to be the centre of the source) is located $18.7 \mathrm{~m}$ from the nose wheel of the aircraft [52], and the center of the engines is $2.91 \mathrm{~m}$ from the ground [53]. The CRJ200 is a newer aircraft, with quieter GE engines; the combination of its takeoff, sideline, and approach noise levels is lower than most other commercial jetliners (they are 77.6, 82.4, and 92.1 EPNdB respectively [52]).

\subsection{Meteorological Conditions}

Measurements took place between 2200 and 2330 on the evening of August 11, 2005. At 2250 , the wind speed was $4.1 \mathrm{~m} / \mathrm{s}$ (within the $5 \mathrm{~m} / \mathrm{s}$ maximum wind speed permitted while taking aircraft noise measurements according to ISO 3891-1978 (E) [54]), from 80; the temperature was $17.1^{\circ} \mathrm{C}$, according to Operations.

The weather in the days leading up to the experiment was pleasant: temperatures were in the high teens/low twenties, the sky was clear to mainly clear, and wind speeds were 


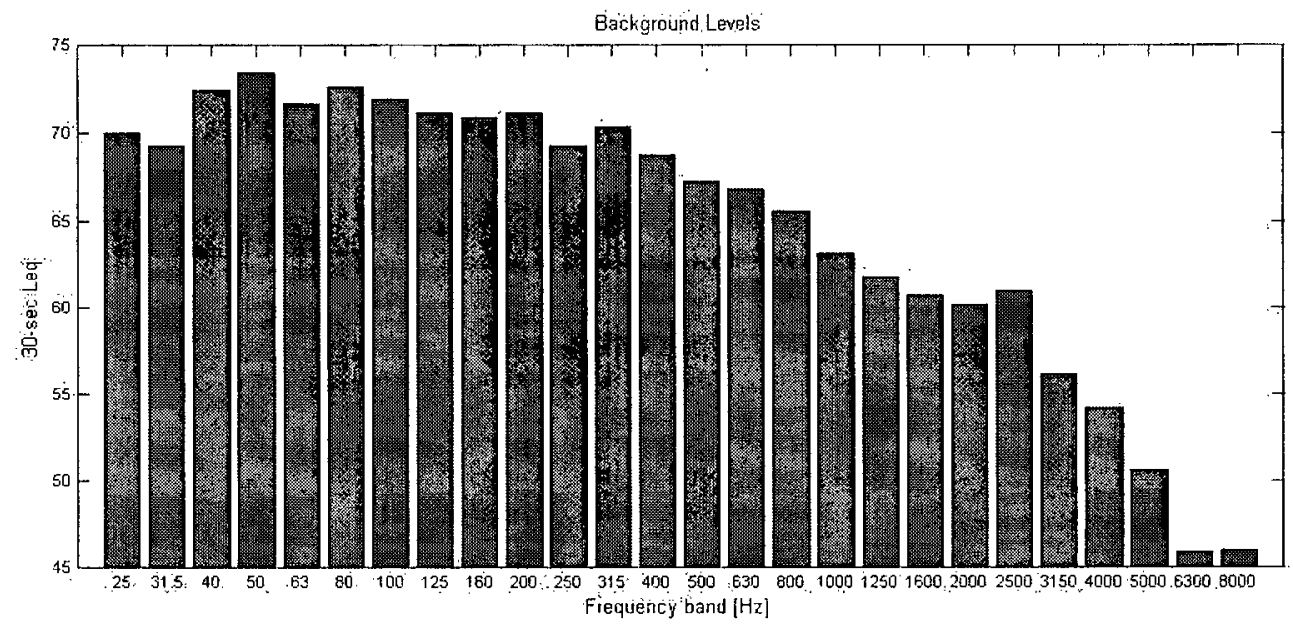

Figure 2.2: Background levels at the run-up site prior to run-up.

typically between $1.4 \mathrm{~m} / \mathrm{s}$ and $4.2 \mathrm{~m} / \mathrm{s}$. August 11 was slightly overcast during the day, but cleared up by the evening. The ground was dry; there was no precipitation the day of the tests, nor had any precipitation accumulated since August 1. The surface over which the measurements took place was relatively flat concrete; variations within the $40 \mathrm{~m}$ radius circle of interest were in the range of $\sim \pm 5 \mathrm{~cm}$. Although difficult to quantify, the wind did not feel particularly gusty according to test personnel who were questioned. There were no major obstacles in the vicinity of the site, and nothing (except the ground) is believed to have perturbed the sound propagating away from the aircraft during the runup. Background noise was estimated to be $\sim 70 \mathrm{~dB}$ while calibrating the equipment; a spectral analysis of a recording performed after calibration but prior to the arrival of the aircraft is illustrated in Figure 2.2 .

\subsection{Equipment}

A few days prior to the noise measurements, a total station - an instrument used by surveyors to measure distances and angles - was used to map out the microphone locations with respect to the nose wheel of the plane. Positions $40 \mathrm{~m}$ from a centre position were marked out in $20^{\circ}$ increments, using water-soluble paint. Two positions along the mid-line indicated where the nose-wheel was to line up, depending on the wind direction (which in turn determines the direction the aircraft must face), such that the exit plane of the engines 
Table 2.1: Equipment used for measurements.

\begin{tabular}{|c|c|c|c|}
\hline Sony PCM-M1 Equipment & First Location & Sccond Location & DAT Recorder \# \\
\hline Larson Davis 2800 & 6 & 7 & 2 \\
\hline Nexus 2690 Conditioning Amplifier & 1 & 0 & 4 \\
\hline RION NA-29E Sound Levcl Meter & 0 & 16 & 5 \\
\hline Quest 155 Sound Level Meter & 17 & 15 & 5 \\
\hline Qucst 2900 Sound Level Meter\#1r & 2 & 4 & 1 \\
\hline Quest 2900 Sound Level Meter\#2j & 3 & 5 & 1 \\
\hline Quest 2900 Sound Levcl Meter\#3k & 11 & 13 & 6 \\
\hline Quest 2900 Sound Levcl Meter\#4m & 12 & 14 & 6 \\
\hline
\end{tabular}

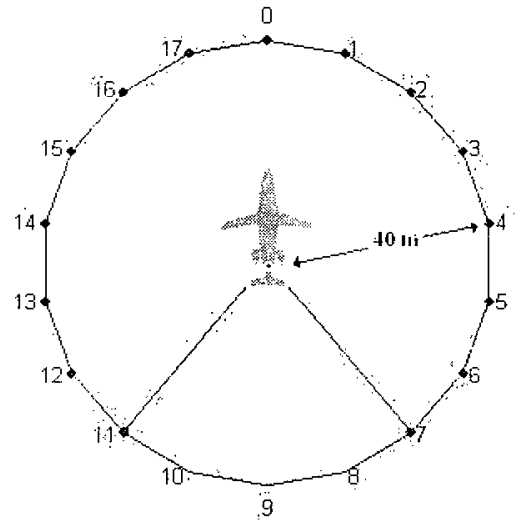

Figure 2.3: Measurements locations for noise measurements.

would be in the center of the circle.

Eight measurement channels were used to record sound pressure levels around the aircraft. The stereo capability of the DAT recorders was exploited to record two signals on one tape in several cases. The equipment used is listed in Table 2.1 and the corresponding locations are illustrated in Figure 2.3. Locations between position 7 and 11 were not measured for safety reasons: it was dangerous to have someone stand in that area of high temperatures and high-speed exhaust. In addition, it was expected that the wind and turbulence created by the exhaust would be high behind the aircraft, and that noise measurements there would be unreliable since air flow over a microphone causes a noise reading.

All sound level meters measuring sound pressure levels were set to flat weighting (A- 
weighting was applied in later analysis), slow response. Half-inch, free-field microphones were used on the sound level meters, each with a windscreen. The DAT recorders were set to sample at $48 \mathrm{kHz}$ to prevent aliasing over the test frequency range, which extended to $11.3 \mathrm{kHz}$. The frequency test range should extend over the same range as the human ear $20 \mathrm{~Hz}$ to $20 \mathrm{kHz}$ - as explained in Chapter 1 . The $11.3 \mathrm{kHz}$ limit was the result of the sound level meters used; the highest frequency they could measure was the upper limit of the 8000 $\mathrm{Hz}$ octave band $\left(8000 \cdot 2^{1 / 2}=11313 \mathrm{~Hz}\right)$. The high frequencies that weren't measured are not of concern for this application: these sound levels are strongly attenuated through atmospheric absorption and are unlikely to reach the community and become problematic. Take for example the third octave bands $6.3,8$, and $10 \mathrm{kHz}$ : the absorption per kilometer is 67,105 , and $157 \mathrm{~dB}$, respectively [2]. The source-receiver distances between run-up pads at YVR and most communities are typically at least $2 \mathrm{~km}$; thus more than $100 \mathrm{~dB}$ of attenuation is expected in each third-octave band.

\subsection{Experimental Procedure}

\subsubsection{Calibration}

Each recording began with $30 \mathrm{~s}$ of a $94 \mathrm{~dB}, 1000 \mathrm{~Hz}$ calibration tone provided by a calibrator. This took place at the run-up pad, prior to the arrival of the aircraft. This tone was later used as the reference for calculating the decibel levels of the measured signals.

\subsubsection{Procedure}

Each sound level meter listed in Table 2.1 was used for four measurements: at two locations (as listed in Table 2.1), and with the microphones held at two heights for each location (1.4 $\mathrm{m}$, and just above the ground, nominally at $0 \mathrm{~m}$ ). These measurements were repeated for three power settings of both engines: idle, 50 percent power, and full power. Each measurement lasted approximately $30 \mathrm{~s}$. The purpose of measuring the sound pressure levels at two heights was to study the effects of ground reflections. It was expected that the sound pressure level would decrease with increasing height above the ground as a result of interference effects. At ground level, the direct and reflected waves are equal in path 
length and phase: they will add constructively, increasing the overall level at the ground by $6 \mathrm{~dB}$ relative to free field. This is due to the pressure doubling right at the ground level. Higher above the ground, the reflected wave will have a longer path length (and may be phase shifted if the ground impedance is finite), and some frequencies will now experience destructive interference due to phase differences between the direct and reflected wave. As a result, sound pressure levels at $1.4 \mathrm{~m}$ above the ground are expected to be lower than sound pressure levels at the ground. Ground and surface waves may also contribute to higher sound pressure levels at the ground as compared with $1.4 \mathrm{~m}$ above the ground.

\subsection{Results}

Problems with equipment limited the total number of recordings. The DAT recorder connected to the Nexus Conditioning Amplifier malfunctioned and did not record anything, and the Quest 2900 Sound Level Meters \#2 and \#4 cut in and out during the measurements (likely due to cable connection problems), limiting the usefulness of their recordings. The informative recordings were played back to a computer where they were digitized by re-sampling at $25 \mathrm{kHz}$ using the GoldWave ${ }^{1}$ audio software program (this high frequency ensured that no aliasing occurred). All Windows volume settings were set to the maximum, leaving one adjustable gain setting in the recording software ("Line In," in GoldWave). All tapes were played back on the same computer and saved as WAV files, with the Line In gain set as high as possible without overload.

MATLAB ${ }^{2}$ was then used to analyze the recordings, using the $94 \mathrm{~dB}, 1000 \mathrm{~Hz}$ calibration tone as the reference for the sound pressure levels. Due to longer computation times, only a section of each $30 \mathrm{~s}$ recording was used. The middle $2,5,10,15$, and $20 \mathrm{~s}$ were analyzed and compared with a randomly chosen $30 \mathrm{~s}$ recording. Little or no difference was found in the results for any of the smaller sections; $5 \mathrm{~s}$ sections were thus analyzed.

Previous measurements of the Dash- 8 and Beechcraft indicated that the directivities of the two aircraft were asymmetrical along the aircraft's centre lines $[13,18]$, a result common to propeller aircraft. Magliozzi et al. [55] describes three types of noise associated with propeller aircraft: steady, unsteady, and random. An example of the former is that,

\footnotetext{
${ }^{1}$ Gold Wave V.5.10, (C2005 GoldWavelnc.

${ }^{2}$ MATLAB V.7.0.0.19920(R14), (C)1984-2004 The MathWorks, Inc.
} 
from the blade passage frequency and its harmonics (this will be steady from the point of reference of a propeller blade rotating). This noise often radiates as a monopole source. Random noise is quite small for propeller aircraft, and is usually neglected. Unsteady noise has asymmetrical directivity - this is the source of the unequal lobes. An example of when unsteady noise occurs is when the propellers are pitched such that the loading of the blades is no longer constant through rotation; this action is commonly taken during run-ups to reduce the lift on the aircraft [55].

Prior to measurement, it was suspected that the directivity of jet aircraft would be symmetric. This was hypothesized since the primary mechanism for noise generation from jet engines is jet noise, which is a broadband source originating from symmetrically shaped jet exhausts located symmetrically on either side of the aircraft. Results from the two sides of the aircraft revealed that this was in fact the case; levels were usually within a couple of $\mathrm{dB}$ of one another - the average difference was $1 \mathrm{~dB}$, and the standard deviation of the difference was also $1 \mathrm{~dB}$ (see Figure 2.4 for comparison of the left and right side of the aircraft). This suggests that, for the locations where measurements were not available, it

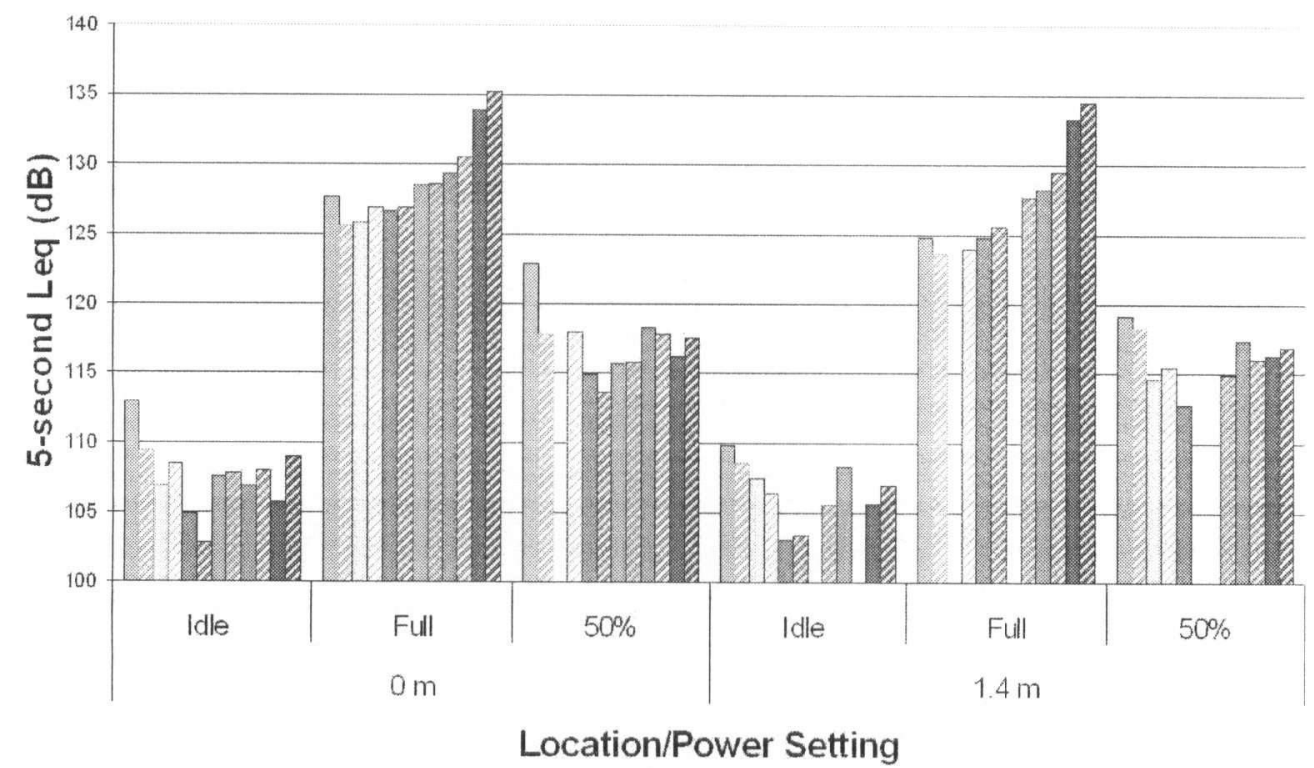

$\square 2: 16 \square 3 \square 15 \square 4: 14 \square 5 \quad 13 \square 6 \quad 12 \square 7 \square 11$

Figure 2.4: Comparison of directivity symmetry of CRJ200. Solid colors are for the right hand side, hatched colors for the corresponding left hand side of the aircraft. 
is acceptable to use the corresponding level on the other side. In so doing, all points were obtained.

Measurements were made at two heights, to see how levels, and the interference of direct and reflected waves, are influenced by the ground effect, surface waves, and ground waves. As expected from the earlier discussion on varying source/receiver heights, levels measured at ground level tended to be higher than those at $1.4 \mathrm{~m}$ above the ground. The differences are plotted in Figure 2.5 .

It is reassuring that the majority of the differences in Figure 2.5 are positive, thus agreeing with theory. For broadband noise, the difference between the sound pressure level at ground level and above ground level should be $3 \mathrm{~dB}$. The average level for these results was only $1.4 \mathrm{~dB}$. Jet noise is primarily broadband, but since it could have some small tonal components, and the sound level meters were not exactly at $0 \mathrm{~m}$ (closer to $10 \mathrm{~cm}$ above ground), a level difference slightly lower than $3 \mathrm{~dB}$ isn't surprising. In a few instances, the sound pressure level at $1.4 \mathrm{~m}$ was higher than at $0 \mathrm{~m}$. This may be a result of the sound level meter not being close enough to the ground, or it may have something to do with experimental uncertainty. The sound level meters, for instance, have an uncertainty of \pm 1 $\mathrm{dB}$ (they were Type I meters). High winds, the inherent uncertainty of the sound level meters, and experimental error (e.g. the exact positions of the sound level meter microphones) all contribute to the uncertainty of the aircraft sound pressure level measurements. This uncertainty will be on the order of $\pm 3 \mathrm{~dB}$. The uncertainty associated with these measurements was relatively small, and for the purpose of prediction, these levels were taken as accurate.

To put the CRJ200 noise levels into perspective, levels measured from the Dash- 8 are plotted in Figure 2.6, and the CR.J200 results in Figure 2.7. Both sets of measurements were done using the same setup (radial distance of $40 \mathrm{~m}$, positions $20^{\circ}$ apart). Sound level meters also recorded levels onto DAT recorders, although some of the sound level meters differed between the two sets of measurements. The only procedural difference between the two was for the $50 \%$ power measurement: only the right engine of the Dash- 8 was at $50 \%$ power (the left idled).

Two major distinctions between the Dash- 8 and the CRJ200 noise measurements are the shapes of the sound pressure level curves, and the magnitude of the levels. The Dash- 8 shows prominent tonal components at lower frequency, particularly at higher powered en- 


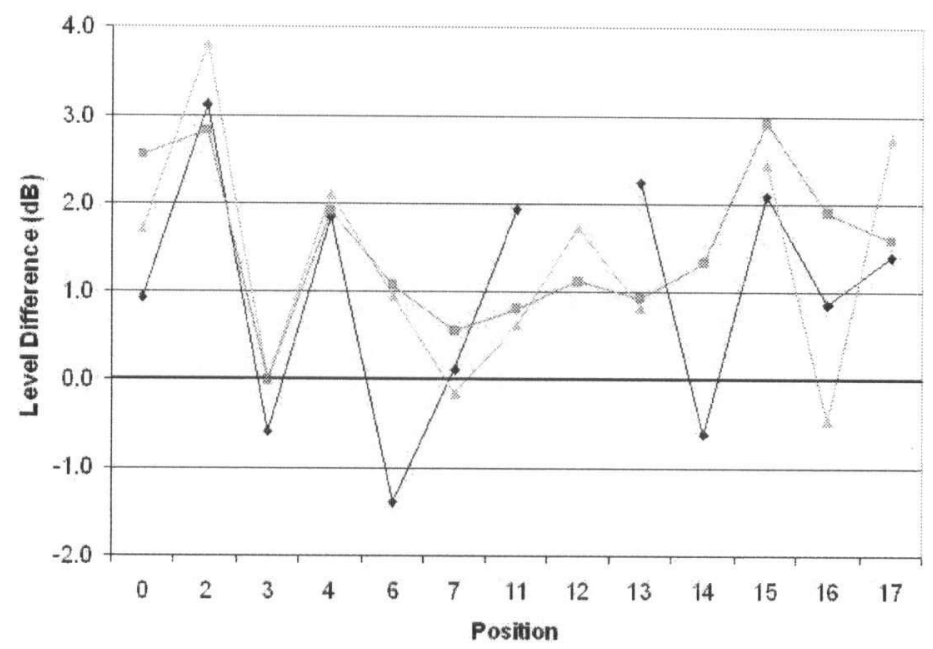

Figure 2.5: Variation with position of difference in $5 \mathrm{~s}$ Leq at two measurement heights. Plotted values are the 0 m level minus the 1.4 m level. (__ ) idle power, (_ ) $50 \%$ power, and ( ) full power.

gine settings. This is related to the propeller noise, and the speed at which the blades are rotating (noise from the blade passage frequency). When more thrust is required for the higher power settings, the propellers rotate more quickly, creating stronger tonal components and higher overall levels. Figure 2.6 shows how these peaks become louder and shift toward higher frequencies as the power level increases and the propeller blades rotate more quickly.

The shapes of the CRJ200 sound pressure level curves are smoother, characteristic of broadband noise - there are no strong tonal components as with the Dash- 8 . The peak at low frequencies, toward the back of the aircraft (e.g. $140^{\circ}$ and $220^{\circ}$ ), and the increase in all levels with power, are likely a result of the increase in exhaust mixing with the atmosphere, required for more thrust.

In addition to the spectral differences, the levels of the CRJ200 tend to be 10-30 dB higher than the Dash-8 sound pressure levels, as expected for a jet vs. propeller aircraft. Sound pressure levels and directivities for the Beechcraft 1900, also a propeller aircraft, were similar to those for the Dash-8. Beechcraft 1900 sound pressure levels have been used for predictions in this work, but as the levels and spectrum are similar to the Dash-8, further comparisons with the CRJ200 would be repetitive and are omitted here. 

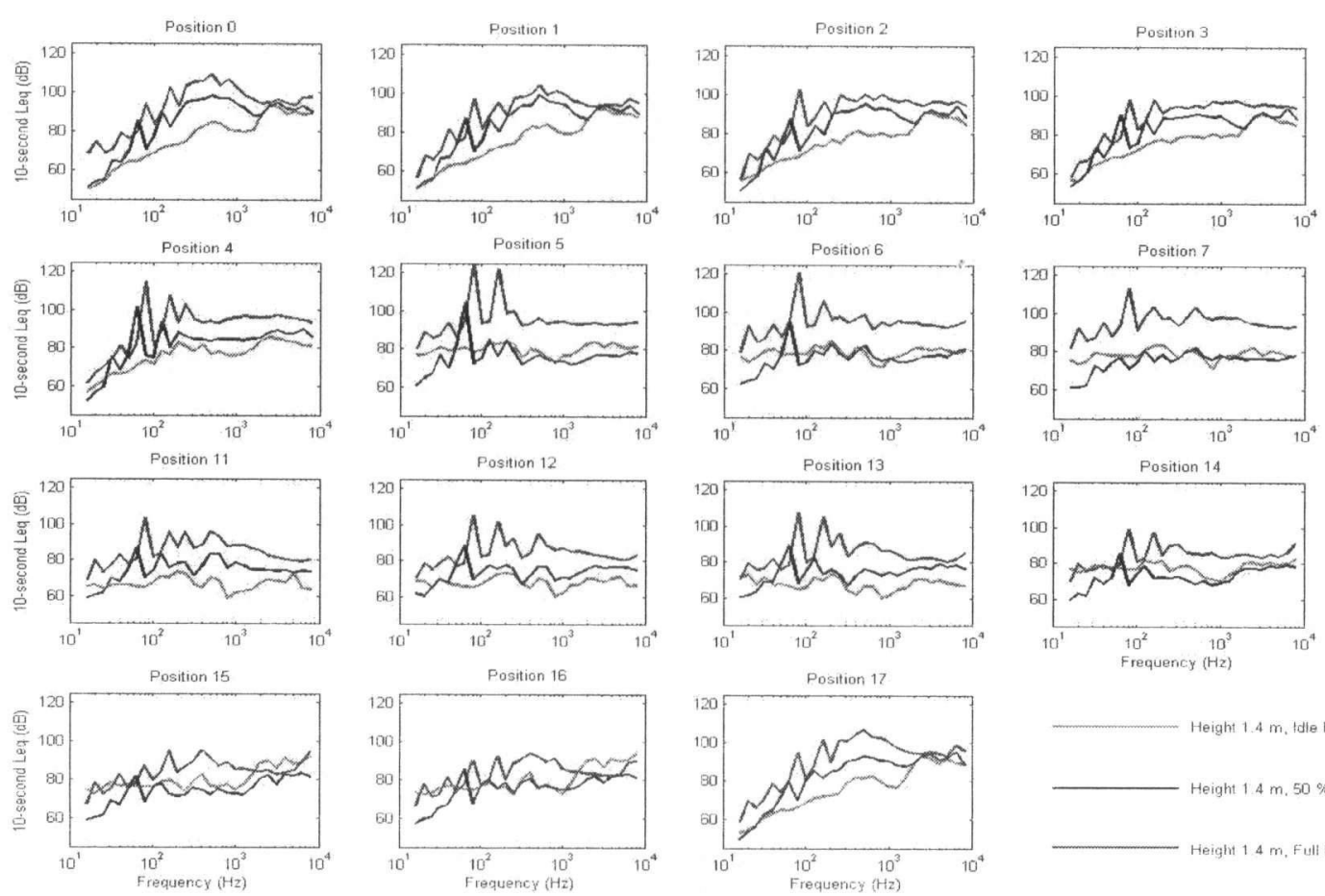

Figure 2.6: Dash 8 Lp at $40 \mathrm{~m}$; degree positions correspond to those in Figure 2.3 [13].
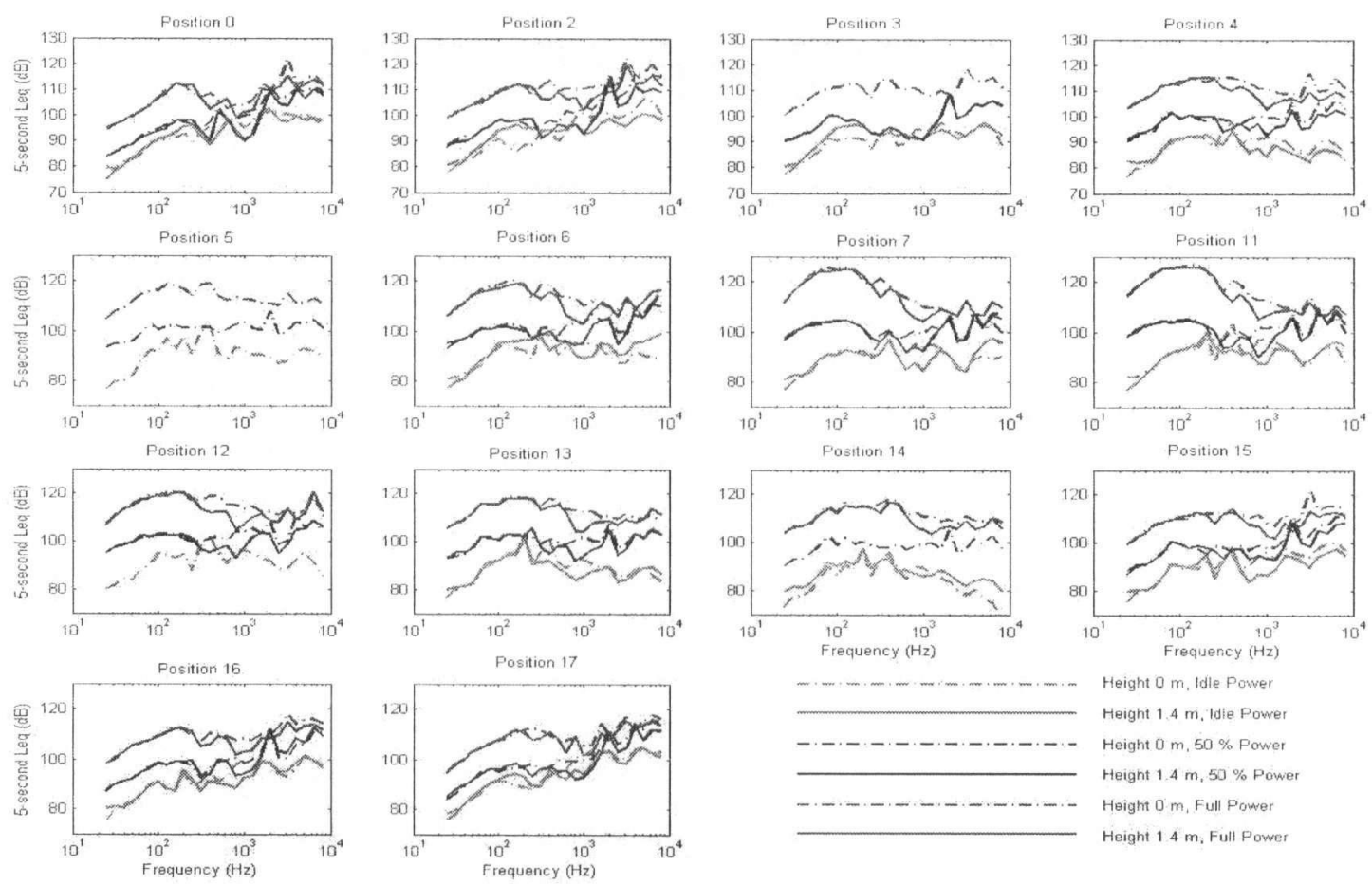

Figure 2.7: CRJ200 Lp at $40 \mathrm{~m}$; degree positions correspond to those in Figure 2.3. 


\subsection{Conclusions}

The noise levels, spectrums, and directivities generated by a small jet aircraft, the CRJ200, at different power settings, were measured. Compared with previous measurements on a Dash-8 propeller aircraft, levels were higher, and spread more equally across all frequencies. Also, strong tonal components were not as apparent as with the Dash-8. The radiation around the CRJ200 appeared to be relatively symmetric about its axis, and evidence of the effect of ground reflections was observed from the measurements taken at different heights.

The GFPE code was used to calculate the attenuation, in terms of transmission loss, from the actual source (the position between the two engines, in the exit plane of the aircraft's engines) to the receiver position. This attenuation was added to the measurements $40 \mathrm{~m}$ away from the source which were discussed in this chapter, and thus the sound pressure levels at $1 \mathrm{~m}$ from the source were available as source data for GFPE predictions. The model used to make the predictions is explained in detail in the following chapter. 


\section{Chapter 3}

\section{Outdoor Sound Propagation}

\section{Modeling and the GFPE Model}

\subsection{Introduction to Models}

There are several outdoor sound propagation prediction models available, each with its own pros and cons. Some of the more common models include the Generalized Fast Field Program (FFP), the Parabolic Equation method (PE), and ray-tracing. There are several ways to solve the parabolic equation numerically; the Crank-Nicholson Parabolic Equation method (CNPE) and the Green's Function Parabolic Equation method (GFPE), are two common methods.

Inherent advantages and disadvantages of these models facilitate and limit their usefulness. FFP models are restricted to environments with layered atmospheres and homogeneous ground surfaces [2]. Ray-tracing programs become extremely computationally expensive for the long ranges involved outdoors, and the appearance of 'caustics' (where rays converge, and an infinite pressure amplitude is predicted rather than a mere increase in level) limit their usefulness [2]. The PE methods assume axisymmetric symmetry and can thus model monopole sources only.

One of the leading criteria for this project was to incorporated mixed ground impedance and, for that reason, the parabolic equation models were explored further. The most apparent difference between the CNPE and the GFPE methods is the step size at which sound is propagated outward from the source (i.e. in the horizontal direction). The horizontal and vertical step sizes for the CNPE are limited to a maximum of $\sim \lambda / 10$ which, for high frequencies and long distances, results in a very large number of steps. While the vertical step size in the GFPE is often recommended to be the same $(\lambda / 10$, or on that order), the horizontal step size is usually between $10 \lambda$ and $100 \lambda$, leading to a major decrease in 
computation time - particularly at higher frequencies $[38,56]$. The step size limitation has to do with how the solution does the calculations between steps: the CNPE follows each oscillation numerically, using a trapezoid integration method, while the GFPE uses the "split-step Fourier algorithm," which is a method that includes the oscillations analytically [57]. As a result, the GFPE can calculate much more quickly than the CNPE $[2,56,57]$.

\subsection{The Original GFPE Code and Required Modifications}

A two-dimensional GFPE program available from previous work [13] has been extended. It was written in Fortran by Xiao Di in 1991 (updated by Di in 1993) to calculate the complex sound pressure as a function of distance over homogeneous ground of arbitrary impedance, with user-defined vertical sound speed gradients [57]. Required changes to this model to achieve the research objectives of this work included:

- modification of the code to account for non-homogenous ground (variations in impedance / ground type with distance);

- frequency-dependent, step size: since many frequencies are used, the constant step size used in the original code is not appropriate for wavelengths from a few centimeters to several meters. Instead, a step size that depends on the wavelength was implemented;

- the addition of air absorption;

- the calculation of the overall A-weighted sound pressure level received at a given distance from a source;

- an option to account for turbulence in prediction; this requires the generation of phase screens for each horizontal and vertical step, for each frequency;

- the addition of a subroutine to calculate the sound speed profile of the source, given temperature and wind conditions, based on typical temperature and wind profiles and/or measurements;

- the prediction of sound pressure levels in third-octave bands (initially, the GFPE calculated levels only for single frequencies); and 
- an estimation of Sound Exposure Level (SEL), and the prediction of the associated annoyance.

Before the details of these modifications can be examined further, it is necessary to explore what the GFPE is, and exactly how it works. The following section will look into these issues.

\subsection{GFPE Theory}

This section describes how the wave equation, which describes sound propagation, is approximated as a parabolic equation and then solved using Green's function. The Green's function used here is the impulse $\exp (i k r) / r$. The major steps in the process begin with the Helmholtz equation, into which sound pressure is substituted for a new, simplified quantity, $q$, which has cylindrical spreading removed. It is then assumed that the majority of sound is propagating in the forward direction and that the wave number is constant across a step $\Delta r$. The Helmholtz equation is subsequently solved by integrating across the small step, $\Delta r$.

The GFPE model solves for sound pressure in a 2D grid $(\mathrm{r}, \mathrm{z})$ : the horizonal dimension is the source-receiver distance, divided by steps $\Delta r$; the vertical dimension is divided into steps $\Delta z$. The domain of the $r$ variable is from the first step $(0+\Delta r)$ to the receiver position. The sound field at $r=0$ is represented by a Gaussian function. The domain of the variable $z$ is from the ground level, where the reflection coefficient determines how much sound is reflected, to an upper absorbing layer, as set by the user. Rather than have the upper atmosphere limit end abruptly, an absorbing layer at least $30 \lambda$ thick exists above the upper limit of the atmosphere through which sound propagates, to prevent sound from reflecting unrealistically back down into the atmosphere.

Unfortunately, direct integration across the steps $\Delta r$ is not possible: instead, the expression is solved for using Green's function, and either spectral representation (as was done here) or using Rayleigh's integral [2, 57]. This process is repeated, iteratively marching through the solution in steps of $\Delta r$, until the receiver sound pressure level is predicted. The following sections describe these steps in more detail. 


\subsubsection{Origins of Sound Equations}

The wave equation can be derived for a small fluid volume for which the following three equations hold:

- the continuity equation; this relates particle velocity, $\vec{u}$, to medium density, $\rho$ (or rather condensation, $s$, where $\left.s=\frac{\rho-\rho_{o}}{\rho_{o}}\right)$ :

$$
\frac{\partial s}{\partial t}+\nabla \cdot \vec{u}=0
$$

The continuity equation describes the conservation of mass;

- the equation of motion, (Euler's equation); this relates the particle velocity to sound pressure, $p$ :

$$
\rho_{o} \frac{\partial \vec{u}}{\partial t}=-\nabla \cdot p
$$

The equation of motion is analogous to Newton's Second Law of Motion $(F=m a)$; and

- the equation of state; this relates sound pressure and density:

$$
p=p_{o} \nu s=B s,
$$

where $\nu$ is the ratio of specific heat at constant pressure to the specific heat at constant

volume $\left(\nu=\frac{c_{p}}{c_{v}}\right), p_{o}$ is the equilibrium pressure, and $B$ is the Bulk modulus of the fluid.

Assuming the fluid (air) through which sound propagates is homogeneous, isotropic, adiabatic, and perfectly elastic, through integration, substitutions, and other manipulations, Equations (3.1), (3.2), and (3.3) can be combined in the more simplified form, the wave equation:

$$
\frac{\partial^{2} p}{\partial t^{2}}=c^{2} \nabla^{2} p
$$

where $c$ is the speed of sound and $\nabla$ is the Laplacian operator [23]. 


\subsubsection{The Helmholtz Equation}

The wave equation can be simplified further. By assuming azimuthal symmetry and harmonic time dependence (i.e. $p=p_{o} \exp (i \omega t)$ ), the corresponding spatial solution in two dimensions becomes:

$$
\left[\frac{\partial^{2}}{\partial r^{2}}+\frac{\partial^{2}}{\partial z^{2}}+k(r, z)^{2}\right] q=0
$$

which is the two-dimensional Helmholtz equation in cylindrical coordinates. Cylindrical spreading has been removed in the above equation by substituting $q(r, z)=p(r, z) \sqrt{r}[2,9$, $57]$.

The solution to the variable $q$ in the Helmholtz equation above (Equation (3.5)) can be written as $q(r, z)=\psi(r, z) \exp i k_{a} r$, where $k_{a}$ is a reference wave number $(e . g ., k(z)$ for a given height) [2]. Substituting this solution into Equation (3.5):

$$
\frac{\partial^{2} \psi}{\partial r^{2}}+2 i k_{a} \frac{\partial \psi}{\partial r}+\frac{\partial^{2} \psi}{\partial z^{2}}+\left(k^{2}-k_{a 1}^{2}\right) \psi=0
$$

The term $\psi$ varies very slowly with $r$ [2], such that Equation (3.6) can be rewritten as:

$$
2 i k_{a} \frac{\partial \psi}{\partial r}+\frac{\partial^{2} \psi}{\partial z^{2}}+\left(k^{2}-k_{a}^{2}\right) \psi=0
$$

In this form, the Helmholtz equation has taken on a structure similar to the parabolic equation. Here instead however, the time derivative has been replaced by $r$, the term that is being 'marched out,' and the spatial variable is simplified to $z$ (this happened at an earlier stage, when axisymmetric symmetry was assumed).

\subsubsection{A more 'realistic' atmosphere: vertical sound-speed profiles}

The discussion thus far have been for the simple case of an unbound, non-refracting atmosphere, where the wave number $k$ is a constant $\left(k=k_{o}\right)$. This is never the situation in real life: $k$ in fact depends on both $r$ and $z$. The wave number $k$ will now be written in height-dependent form: $k=k(z)=w / c(z)$. Physically, this means that effects such as temperature inversions/lapses and wind, which cause sound to be refracted, can be modeled with a sound-speed profile. If the range dependence of the wave number is weak enough, such that it can be neglected over a step $\Delta r$, the r-dependence can be neglected $[2,9]$. In 
this case, an exact solution to the parabolic equation exists. Separating the $z$-dependent parts of Equation (3.5),

$$
\frac{\partial^{2} q(r, z)}{\partial r^{2}}=-\left(k^{2}(z)+\frac{\partial^{2}}{\partial z^{2}}\right) q(r, z)
$$

and simplifying by substituting $H(z)=k^{2}(z)+\frac{\partial^{2}}{\partial z^{2}}$,

$$
\frac{\partial^{2} q(r, z)}{\partial r^{2}}=-H(z) q(r, z)
$$

By observation, two solutions to (3.9) exist, corresponding to a forward and a backward traveling wave. Assuming sound is primarily propagating in the forward direction (i.e. in one direction away from the source, with minimal backscatter - this is an important assumption), the wave traveling backward is neglected and only the forward solution is used:

$$
\frac{\partial q(r, z)}{\partial r}=i H^{\frac{1}{2}}(z) q(r, z)
$$

Since it is of interest explore how the speed of sound varies with height, the wave number in Equation (3.8) will be split, into constant and varying terms $\left(k^{2}(z)=k_{o}^{2}+\delta k^{2}(z)\right)$, allowing $H$ to be rewritten as:

$$
H(z)=k_{o}^{2}+\delta k^{2}(z)+\frac{\partial^{2}}{\partial z^{2}},
$$

where $k_{o}$ is some constant reference wave number (e.g. at ground level), and $\delta^{2} k(z)$ is a function of height which corrects for the vertical sound-speed profile. How $H$ is solved for in Equation (3.10), and the approximations/assumptions made in doing so, determine what type of parabolic equation solution is being applied (e.g. Crank Nicholson, which uses a "rational Padé expansion"' [15], or the Green's Function, which uses the Green's functions and a split step approximation (15]). We will continue with the latter.

Assuming $\delta k(z)$ is small compared with the other terms, the square root of $H$ may be approximated as $[2,57]$ :

$$
H^{1 / 2}(z) \simeq \sqrt{k_{o}^{2}+\frac{\partial^{2}}{\partial z^{2}}}+\frac{\delta^{2} k(z)}{2 k_{o}} .
$$

Substituting back into the one-way solution:

$$
\frac{\partial q(r, z)}{\partial r}=i\left(\sqrt{k_{o}^{2}+\frac{\partial^{2}}{\partial z^{2}}}\right) q(r, z)+i \frac{\delta^{2} k(z) q(r, z)}{2 k_{o}} .
$$


The first term on the right hand side describes the non-refracting part of the atmosphere (note that it only depends on $k_{o}$ ), and the second term on the right hand side corrects for the effect of atmospheric refraction (note the $z$-dependence of $k$ ).

The marching step solution can be obtained from 3.13 by integrating from $r$ to $r+\Delta r$ such that:

$$
q(r+\Delta r, z)=\exp \left(i \Delta r \frac{\delta k^{2}(z)}{2 k_{o}}+i \Delta r \sqrt{k_{o}^{2}+\frac{\partial^{2}}{\partial z^{2}}}\right) q(r, z)
$$

If it is assumed that $\sqrt{k_{o}^{2}+\frac{\partial^{2}}{\partial z^{2}}}$ and $\frac{\delta k^{2}(z)}{2 k_{o}}$ commute, Equation (3.14) can be split into two exponential terms:

$$
q(r+\Delta r, z)=\exp \left(i \Delta r \frac{\delta k^{2}(z)}{2 k_{o}}\right) \exp \left(i \Delta r \sqrt{k_{o}^{2}+\frac{\partial^{2}}{\partial z^{2}}}\right) q(r, z)
$$

Gilbert et al. explain that the first part in Equation (3.15) is simply a phase factor, correcting for refraction; the second term is the solution for the non-refracting, homogeneous atmosphere. The 'split Fourier step' name comes from this part: according to Equation (3.15), q can be solved for a homogeneous atmosphere until the last step, where the refraction factor is finally multiplied in. The assumptions made in arriving at this solution, where the phase factor describes the variations in vertical sound speeds, are only valid when $\delta k^{2} \ll k_{o}^{2}[57]$.

It is, however, difficult to solve for $q$ in this form, due to the implicit $z$-dependence of $H$. Several numerical methods exist to solve such a problem. One method involves the Kirchhoff-Helmholtz integration method, which uses Green's functions and boundary conditions to arrive at an equation for the direct, reflected, and surface-wave propagation [2]. Gilbert and Di (the original authors of the GFPE model used in this work) alternatively use a method whereby the operator $H$ is rewritten explicitly using 'spectral representation' [57] and Green's functions.

According to Gilbert et al., the spectral representation of a general function of an operator $Q$ can be expressed as [57]:

$$
F(Q)=\frac{1}{2 \pi i} \int_{C} \frac{F(x)}{\tau-Q} d \tau
$$

where the integral of the contour $C$ encloses the eigenvalues $\tau$.

For the function, $q(r+\Delta r)=\exp (i \Delta r \sqrt{H})$, substituting $\kappa^{2}=x$ (thus $d x=2 \kappa d \kappa$, where 
$\kappa$ is the horizontal wave number, $k(z)=\kappa+k_{z}$ ), Equation (3.16) becomes:

$$
F(H)=\frac{1}{\pi i} \int_{C} \frac{e^{i \Delta r \kappa}}{I \kappa^{2}-H} q(r, z) d \kappa .
$$

The Green's operator that solves $\left(H-\kappa^{2} I\right) G(\kappa)=-I$, where $I$ is the identity matrix, is:

$$
G(\kappa)=\left(\kappa^{2} I-H\right)^{-1} .
$$

Green's functions are mathematical methods used to solve inhomogeneous ordinary differential equations with unique solutions based on boundary conditions [58]. Green's functions are defined by:

$$
\tilde{L} G(\vec{r}, \vec{r})=\delta\left(\vec{r}-\vec{r}^{\prime}\right)
$$

where $\tilde{L}$ is a linear operator.

Substituting Equation (3.18) into Equation (3.17):

$$
q(r+\Delta r)=\frac{1}{\pi i} \int_{C} G(\kappa) e^{i \Delta r \kappa} \kappa d \kappa
$$

or with explicit $z$-dependence:

$$
q(r+\Delta r, z)=\frac{1}{\pi i} \int_{C} e^{i \kappa \Delta r} \kappa d \kappa \int_{0}^{\infty} G\left(\kappa, z, z^{\prime}\right) q\left(r, z^{\prime}\right) d z^{\prime} .
$$

Gilbert et al. find $G\left(\kappa, z, z^{\prime}\right)$ such that it satisfies $\left(\frac{\partial^{2}}{\partial z^{2}}+k^{2}(z)-\kappa^{2}\right) G\left(\kappa, z, z^{\prime}\right)=$ $-\delta\left(z-z^{\prime}\right)$ for sound propagation over an impedance boundary to be [57]:

$$
G\left(\kappa, z, z^{\prime}\right)=\frac{1}{2 k_{v}}\left[\exp \left(i k_{v}\left|z-z^{\prime}\right|\right)+R\left(k_{v}\right) \exp \left(i k_{v}\left(z+z^{\prime}\right)\right)\right]
$$

where

$$
R\left(k_{v}\right)=\frac{k_{v} Z_{g}-k_{o}}{k_{v} Z_{g}+k_{o}}
$$

and where $Z_{g}$ is the normalized ground impedance. With some rearrangement, and using some identities, Equation (3.20) can be rewritten as [2, 57]: 


$$
\begin{aligned}
& q(r+\Delta r, z)=\exp \left(\frac{i \Delta r \delta k^{2}}{2 k_{r}}\right) \\
& *\left(\frac{1}{2 \pi} \int_{-\infty}^{\infty} \exp \left(i \Delta r \sqrt{k_{o}^{2}-k^{\prime 2}}\right) e^{i k^{\prime} z} d k^{\prime} \int_{o}^{\infty} e^{-i k^{\prime} z^{\prime}} q\left(r, z^{\prime}\right) d z^{\prime}\right. \\
& +\frac{1}{2 \pi} \int_{-\infty}^{\infty} R\left(k^{\prime}\right) \exp \left(i \Delta r \sqrt{k_{o}^{2}-k^{\prime 2}}\right) e^{i k^{\prime} z} d k^{\prime} \int_{0}^{\infty} e^{i k^{\prime} z^{\prime}} q\left(r, z^{\prime}\right) d k^{\prime} \\
& \left.+2 i \beta e^{-i \beta z} \exp \left(i \Delta r \sqrt{k_{o}^{2}-\beta^{2}}\right) \int_{0}^{\infty} e^{-i \beta z^{\prime}} q\left(r, z^{\prime}\right) d z^{\prime}\right)
\end{aligned}
$$

where $\beta=k_{o} / Z_{g}$. Recall that the term $q$ was the sound pressure with spherical spreading removed.

Each of the three terms on the right hand side of Equation (3.24) has a real, physical meaning: the first represents the direct wave, the second represents the reflected wave, and the third represents the surface wave. These - particularly the former two - are the main contributors to the sound pressure level at receiver positions. .

\subsubsection{Refraction}

The refraction of sound can be implemented in different ways. In the previous sections, for Equation (3.15), the refraction factor was derived to be:

$$
\exp \left(i \Delta r \frac{\delta k^{2}(z)}{2 k_{o}}\right)
$$

According to Ref. [2], a more accurate prediction can be obtained by assuming $\delta k(z)=$ $k(z)-k_{o}$ instead of $\delta k^{2}(z)=k^{2}(z)-k_{o}^{2}$; this results in the following refraction factor instead of Equation (3.25):

$$
\exp (i \Delta r \delta k(z))
$$

The original GFPE code has a flag allowing the user to select which refraction factor to use. For this work, Equation (3.26) was chosen. 


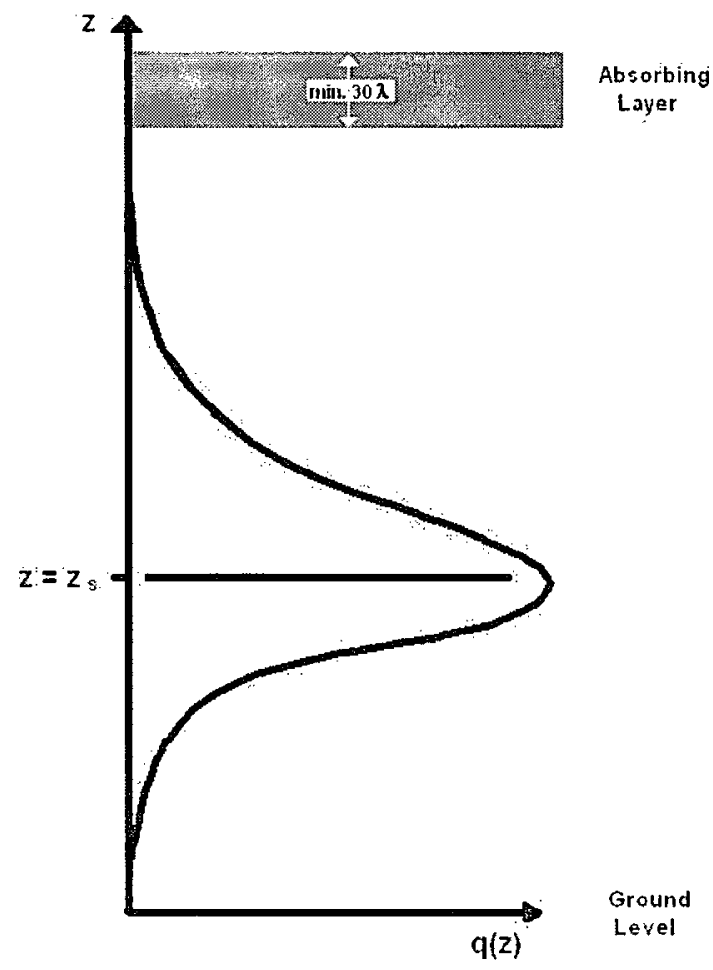

Figure 3.1: Gaussian starting function [14].

\subsubsection{Starting Field}

The GFPE is a long-range solution and, as such, it cannot be implemented directly at the source. Instead, a starting field must be used. The standard expression for a monopole point source is $[2,57]$ :

$$
q(r, z)=\frac{\exp (i k R)}{R} \sqrt{r}
$$

where $R$ is the radial distance from the source. This cannot be used for the starting field, because it diverges very close to the source, and fails at larger elevation angles $[2,57]$.

Gaussian starting fields are common with the GFPE: one can imagine this as a horizontal Gaussian curve, centered at the source height, and spreading to zero at the ground level and at the absorption layer height, as illustrated in Figure 3.1 [2]. Gilbert et al. express the Gaussian starting field as:

$$
q(r=0, z)=\left[\exp \left(-\left(\frac{z-z_{s}}{2 / k_{o}}\right)^{2}\right)+\frac{Z_{g}-1}{Z_{g}+1} \exp \left(-\left(\frac{z+z_{s}}{2 / k_{r}}\right)^{2}\right)\right] \sqrt{\frac{k_{r}}{2}}
$$




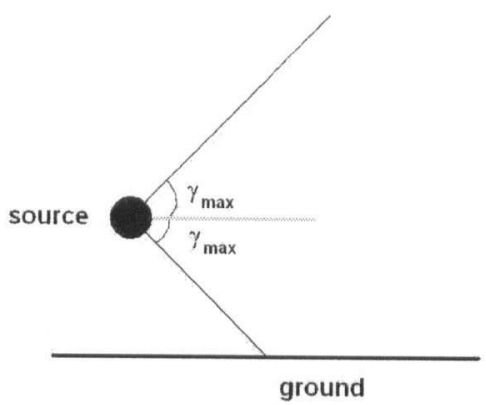

Figure 3.2: Elevation angle limitation: between angles $-\gamma_{\max }$ and $\gamma_{\max }$, the PE model is valid [2].

This is the starting field Di used in the GFPE model used in this work.

Gaussian functions are good approximations of cylindrical and spherical sources; they are usually used as the starting field in parabolic equation models $[2,14,57]$.

\subsubsection{Elevation Angle Limitation}

One limitation of $\mathrm{PE}$ methods is the limited elevation angle for which they can accurately predict. It is assumed that, as long as the receiver is within the $-\gamma_{\max }$ to $\gamma_{\max }$ range (see Figure 3.2), correct results can be expected. According to test simulations, GFPE is valid up to much higher elevation angles than the CNPE [2]. The elevation angle for the GFPE is somewhere between $30^{\circ}$ and $60^{\circ}$ (depending on the starting field) [2]. For the source/receiver heights used in the present work (the heights of the engines, of bedrooms in houses, etc.) and the larger distances between sources and receivers (several kilometers), predictions are well within the region of accurate predictions.

\subsection{Modifications to GFPE}

The GFPE model used in this work was rather basic to start with. Modifications to the code (as discussed at the beginning of this chapter) were made, in order predict A-weighted sound levels more accurately. The GFPE is written in FORTRAN; the original version has approximately ten subroutines, an input file, and two flags. The following sections describe 
the modifications in detail - what subroutines were changed, what subroutines were added - as well as the changes made to the main GFPE program file (Main.f).

\subsubsection{Variable Grounds}

The GFPE has one large advantage over many other modeling methods: it can accommodate range-dependent phenomena. As described earlier, and as shown in Equation (3.24), the GFPE models three waves: the direct, the reflected, and the surface waves. The type of ground is essential for the latter two waves: the reflected wave, since the reflection coefficient depends on the ground impedance, and the surface wave, since it only propagates over finite impedance surfaces $[2,38,57]$.

The improved GFPE model allows the user to select up to six different ground types along the source/receiver line in a given simulation, each separated by a 'transition point.' Additional transition points can be added if required for future work. Furthermore, six predefined, finite-ground impedances were programmed into the GFPE model, based on what was considered typical for the areas at and around YVR. A recent study involving soil sampling for an expansion project at YVR revealed that the topsoil was composed primarily of silt to silt loam (a mixture of sand, silt, and clay) [59]. According to a list, of ground types provided by Attenborough [1] ${ }^{1}$, Loamy Sand Beneath Lawn (both wet and dry) was the closest match. Fine grain sand was also selected (wet and dry), along with snow (for the occasional Vancouver winter day). In addition to the finite ground types, a sixth, infinite ground impedance surface was also included. Infinite impedance represents acoustically hard surfaces, such as water, concrete, and asphalt. Details of the parameters used for the calculations are listed in Table 3.1.

The acoustical impedances of the five finite impedance surfaces listed above were calculated based on Attenborough's 2-parameter model, described in Section 1.2.3. Additional ground impedances can be added.

The values for the frequency-dependent, finite ground impedances are listed in the new subroutine 'IMP.f.' For each frequency and at each distance between transition points, this subroutine assigns the appropriate impedance value.

\footnotetext{
${ }^{1}$ Keith Attenborough is one of the leading researchers on ground impedance, having developed the models used today including the 4-parameter model, and the 2-parameter model used in this work.
} 
Table 3.1: Parameters used for finite ground impedance calculations. 0.5 was used for the pore shape factor ratio in all cases [1].

\begin{tabular}{|c|c|c|}
\hline Ground Type & Flow Resistivity $\left(\mathrm{kPa} \mathrm{s} \mathrm{m}^{-1}\right)$ & Porosity \\
\hline Loamy sand beneath lawn (dry) & 677000 & 0.288 \\
\hline Loamy sand beneath lawn (wet) & 677000 & 0.137 \\
\hline Loamy sand on plain (dry) & 422000 & 0.375 \\
\hline Loamy sand on plain (wet) & 422000 & 0.112 \\
\hline Snow & 473 & 0.86 \\
\hline
\end{tabular}

\subsubsection{Step Size}

Initially, the horizontal and vertical step sizes were defined in the input file as constant values. Since the GFPE is to predict levels over several octave bands, a step size that depends on wavelength is more appropriate. To model sound propagation, the horizontal step size should be between $\sim 5-10 \lambda$ and $\sim 50-100 \lambda[38,56]$. Smaller steps sizes increase the computational time and can introduce numerical errors [56]. Larger step sizes will bypass finer details in the sound field that may be critical for an accurate representation.

It is recommended in the literature to use a vertical step size on the order of $\lambda / 10$. This was achieved by removing the variable $d z$ from the original INPUT file and placing it in the subroutine GEOM.f. This small step size has a substantial effect on the size, $N$, of the Fourier transform. Recall that:

- $N$ is the number of vertical steps, which include the atmosphere, and the absorbing layer above of at least $30 \lambda$ (again, this is used to prevent unrealistic reftections down from the top height set in the model); and

- $N$ should be a power of 2 , to take advantage of the Fast Fourier transform.

A very large value for $N$ is needed to properly model the required number of vertical steps, particularly at higher frequencies and in situations where the absorbing layer is higher above the ground. 


\subsubsection{Multiple Frequencies}

With the original GFPE code, the sound pressure of a single frequency sound was predicted, rather than a total level representing the complete audible frequency range. The code specifically MAIN.f - was modified to repeatedly run through the simulation for a range of frequencies. After each run, the frequency is multiplied by $2^{1 / 3}$ - thus calculating levels for the centre frequencies of third-octave bands. While the frequency interval could be adjusted, an assumption was made that the energy at the centre frequency of a third-octave band represented the total energy in that band. The centre frequencies for third-octave bands from 25 to $4000 \mathrm{~Hz}$ were defined in the new model. This range was chosen due to:

- A-weighting decreasing sound pressure levels at the lower frequency limit; and

- air absorption decreasing sound pressure levels at the upper frequency limit.

These two factors are discussed in the next section.

\subsubsection{Corrections: A-Weighting, Air Absorption}

The upper and lower frequency limits were chosen as a result of two required corrections: the former for air absorption, and the latter for A-weighting. Low frequency sound of a given sound pressure level is perceived as being much quieter compared to mid- and highfrequencies. The correction at low frequencies is so large, that the low frequency third-octave band sound pressure levels have a negligible effect. Recall that third-octave band sound pressure levels are added using decibel addition. Air attenuation has a similar effect, but on high frequencies rather than low.

A-weighted levels are of interest here, since we are concerned with the human perception of noise. Values for the A-weighting corrections are listed in Table 3.2. They were calculated based on the International Standard IEC 651 (1993-09) [2].

Atmospheric absorption depends on temperature, relative humidity, and the ambient pressure. The values input for this work are from Ref. [2]; they are presented in Table 3.3.

When calculating the attenuation caused by air absorption, it was assumed that the path traveled by the sound from source to receiver was a straight, direct path. This is inaccurate: due to refraction, the paths curve and are longer, the addition in length depending on the curvature of the arc that the sound wave forms. This can be described quantitatively 
Table 3.2: A-weighting correction for 1/3 octave bands, according to IEC 651 (1993-09) [2]

\begin{tabular}{|c|c|c|c|c|c|c|c|}
\hline Frequency $(\mathbf{H z})$ & 25 & 31.5 & 40 & 50 & 63 & 80 & 100 \\
\hline $\mathbf{W}_{A}$ & -44.8 & -39.5 & -34.5 & -30.3 & -26.2 & -22.4 & -19.1 \\
\hline \hline Frequency $(\mathbf{H z})$ & 125 & 160 & 200 & 250 & 315 & 400 & 500 \\
\hline $\mathbf{W}_{A}$ & -16.2 & -13.2 & -10.8 & -8.7 & -6.6 & -4.8 & -3.2 \\
\hline \hline Frequency $(\mathbf{H z})$ & 630 & 800 & 1000 & 1250 & 1600 & 2000 & 2500 \\
\hline $\mathbf{W}_{A}$ & -1.9 & -0.8 & 0.0 & 0.6 & 1.0 & 1.2 & 1.3 \\
\hline
\end{tabular}

Table 3.3: Attenuation in third-octave bands resulting from atmospheric absorption. Values are listed in $d B / \mathrm{km}$, for temperature of $283.15 K$, relative humidity of $80 \%$, and atmospheric pressure of $1.01325 \times 10^{5} \mathrm{~Pa}[2]$.

\begin{tabular}{|c|c|c|c|c|c|c|}
\hline $25 \mathrm{~Hz}$ & $31.5 \mathrm{~Hz}$ & $40 \mathrm{~Hz}$ & $\mathbf{5 0 ~ H z}$ & $\mathbf{6 3} \mathrm{Hz}$ & $80 \mathrm{~Hz}$ & $100 \mathrm{~Hz}$ \\
\hline 0.018 & 0.028 & 0.045 & 0.07 & 0.11 & 0.17 & 0.25 \\
\hline \hline $125 \mathrm{~Hz}$ & $\mathbf{1 6 0 \mathrm { Hz }}$ & $\mathbf{2 0 0 \mathrm { Hz }}$ & $\mathbf{2 5 0 \mathrm { Hz }}$ & $\mathbf{3 1 5} \mathrm{Hz}$ & $\mathbf{4 0 0 \mathrm { Hz }}$ & $\mathbf{5 0 0 ~ H z}$ \\
\hline 0.37 & 0.55 & 0.77 & 1.02 & 1.31 & 1.63 & 1.96 \\
\hline \hline $630 \mathrm{~Hz}$ & $\mathbf{8 0 0 \mathrm { Hz }}$ & $\mathbf{1 0 0 0 \mathrm { Hz }}$ & $\mathbf{1 2 5 0 \mathrm { Hz }}$ & $\mathbf{1 6 0 0 \mathrm { Hz }}$ & $\mathbf{2 0 0 0 \mathrm { Hz }}$ & $\mathbf{2 5 0 0 \mathrm { Hz }}$ \\
\hline 2.36 & 2.88 & 3.57 & 4.58 & 6.3 & 8.8 & 12.6 \\
\hline
\end{tabular}

for a linear sound speed profile by:

$$
R=\frac{c}{\left|\frac{d c}{d h}\right|}
$$

where $\mathrm{c}$ is the speed of sound, $\mathrm{h}$ is the height in the atmosphere, and $\mathrm{R}$ is the radius of curvature of sound [60]. In a downward refracting atmosphere with a linear sound speed gradient, the path length of sound is approximately 1.5 times greater $(1570 \mathrm{~m}$ at $1 \mathrm{~km}, 7854$ $\mathrm{m}$ at $5 \mathrm{~km}$, and $15708 \mathrm{~m}$ at $10 \mathrm{~km}$ ). Table 3.4 shows the change in attenuation resulting from a curved path at selected frequencies. These distances are significantly longer, and while the associated additional atmospheric absorption at low frequencies is quite small, the results in Table 3.4 confirm the validity of the high frequency limit in GFPE calculations.

The values in Tables 3.2 and 3.3 have been input into the subroutine MAIN, where 
Table 3.4: Comparison of attenuation [in $\mathrm{dB}$ ] resulting from a linear sound speed gradient at select frequencies and source-receiver distances

\begin{tabular}{|c|c|c|c|}
\hline Frequency $(\mathrm{Hz})$ & $1 \mathrm{~km}(1570 \mathrm{~m})$ & $5 \mathrm{~km}(7854 \mathrm{~m})$ & $10 \mathrm{~km}(15708 \mathrm{~m})$ \\
\hline 25 & $0.02(0.03)$ & $0.09(0.14)$ & $0.18(0.28)$ \\
\hline 63 & $0.11(0.17)$ & $0.55(0.86)$ & $1.10(1.73)$ \\
\hline 500 & $1.96(3.08)$ & $9.8(15.4)$ & $19.6(30.8)$ \\
\hline 1000 & $3.6(5.6)$ & $17.9(28.0)$ & $35.7(56.1)$ \\
\hline 2500 & $12.6(19.8)$ & $63.0(99.0)$ & $126(198)$ \\
\hline
\end{tabular}

they are included in the attenuation.

\subsubsection{Turbulence}

Turbulence is included in predictions as small fluctuations in the sound speed. This is equivalent to multiplying the complex sound pressure level by a phase factor. The phase factors are generated semi-randomly, and are stored in arrays to later be used with the GFPE code. These arrays are often called 'phase screens.'

Rather than calculating the sound speed fluctuations directly, however, it has been found that the Fourier transform of these phase values can be generated more easily using frequency spectrums. Three of the more common spectrums in atmospheric acoustics are the Gaussian, the von Kármán, and the Kolmogorov spectrums. These three spectrums have been listed from the simplest to most complicated to generate, and also from the least to most accurate. Early work on turbulence normally used the Gaussian spectrum; more recent work has used the latter two [2].

Turbulence depends on both temperature and wind fluctuations; the spectrums mentioned above can account for one or both types of fluctuation. The same problem, however, occurs as with the ground impedance; the more accurate you try to make your results, the more difficult it becomes to know each of the inputs with sufficient accuracy. If the inputs are unknown, it may be best to use a simpler model, rather than to guess incorrectly at the values of certain parameters.

The two-dimensional von Kármán spectrum for temperature fluctuations has been used in this work. It is defined as [2]: 


$$
F(k)=\mu_{o}^{2} \frac{\Gamma(8 / 6)}{\Gamma(1 / 3) \pi} \frac{a^{2}}{\left(1+k^{2} a^{2}\right)^{8 / 6}}
$$

where $\Gamma$ is the Gamma function $\left(\Gamma(z)=\int_{0}^{\infty} t^{z-1} e^{-t} d t\right), \mu_{o}$ is a standard deviation proportional to the standard deviation of temperature $\left(\mu_{o}{ }^{*}=\frac{1}{2} \sigma_{T} / T_{o}\right.$, where $\sigma_{T}$ is the standard deviation of temperature and $T_{o}$ is the mean value) - this is a measure of the intensity of turbulence [16], $k$ is the wave number, and $a$ is the correlation length. Correlation lengths are typically around $1 \mathrm{~m}$, and $\mu_{o}^{2}$ is usually between $10^{-6}$ and $10^{-5}[2,9]$.

Salomons [2] explains that, in models where the outgoing step size is small - for instance the CNPE - the sound pressure is multiplied by $\exp \left(i k_{a} \mu \Delta r\right)$. Another approach is necessary for the larger steps taken in the GFPE: for larger $r$, integration must be done over $\mu$, which depends on $r$. In the GFPE, the phase screen is represented by:

$$
\exp (i \Theta)
$$

where

$$
\Theta=k_{z} \int_{r}^{r+\Delta r} \mu(r, z) d r
$$

According to Salomons [2], Equation (3.32) can be approximated by:

$$
\Theta=\Upsilon\left(r+\Delta r, z_{j}\right)-\Upsilon\left(r, z_{j}\right)
$$

where

$$
\Upsilon\left(r, z_{j}\right)=k_{a} \sum_{n} \frac{G\left(\mathbf{k}_{n}\right)}{k_{n r}} \sin \left(r k_{n i r}+z_{j} k_{n z}+\alpha_{n}\right),
$$

and where $\alpha_{n}$ is a random number between 0 and $2 \pi$, and:

$$
G\left(\mathbf{k}_{n}\right)=\sqrt{4 \pi \Delta k F\left(\mathbf{k}_{n}\right)},
$$

where $k_{n r}=k_{n} \cos \left(\theta_{n}\right)$ and $k_{n z}=k_{n} \sin \left(\theta_{n}\right), \mathbf{F}\left(\mathbf{k}_{n}\right)$ is given by Equation (3.30), and $\theta_{n}$ is chosen randomly between 0 and $2 \pi[2]$.

MATLAB was used to generate phase screens prior to the GFPE simulations. An option in the input file allows the user to choose whether turbulence should be included or not. The phase screen multiplies the complex pressure (or rather $q$ ) to produce the turbulent 
atmosphere predictions.

\subsubsection{Sound Speed Profile}

The speed of sound in a gas is:

$$
c_{g a s}=\sqrt{\nu R T}
$$

where $\nu$ is the adiabatic index ( $\sim 1.4$ for air), $R$ is the gas constant, and $T$ is the absolute temperature (in Kelvin). $R$ defined as the universal gas constant $\left(8.3144 \mathrm{~J} \mathrm{~K}^{-1} \mathrm{~mol}^{-1}\right.$ ) divided by the molar mass of air $(28.97 \mathrm{~g} / \mathrm{mol})$, and equals $287.05 \mathrm{~J} \mathrm{~kg}^{-1} \mathrm{~K}^{-1}$.

The effects of humidity on sound speed are understood and can be included; however, since the change in sound speed is less than $0.7 \%$ over the range of 0 to $100 \%$ relative humidity, its effect was considered negligible in this work [61]. The effect of wind on the speed of sound is straightforward: it is included by vector addition with the sound speed.

Five new input parameters have been added to the input file, along with the new subroutine CPROF.f, which together compute the variation of the speed of sound with height. The input parameters are: the temperature at $6 \mathrm{~m}$, the wind speed at $10 \mathrm{~m}$, the wind direction, the source-receiver direction, and the choice of either a neutral or stable atmosphere. By selecting a stable atmosphere, a parabolic temperature profile is calculated, which assumes the temperature decreases by $2^{\circ} \mathrm{C}$ at the ground surface and increases by $6^{\circ} \mathrm{C}$ at $200 \mathrm{~m}$ above the ground. The neutral atmosphere selection uses a linear profile where temperature decreases with height at a rate of $0.0098^{\circ} \mathrm{C} / \mathrm{m}$.

The wind profile power law,

$$
\frac{u(z)}{u(10)}=\left(\frac{z}{10}\right)^{1 / 7},
$$

where $u$ is wind speed and $z$ is height above the ground, was used calculate the variation of wind speed with height. This is commonly used to approximate wind profiles in the absence of information other than the sound speed at a $10 \mathrm{~m}$ height. The wind vector was then projected onto the source-receiver direction and added to the sound speed. 


\subsubsection{Sound Exposure Level (SEL)}

In Chapter 1, sound exposure level was explained to be a measure of an event's energy compressed or expanded into $1 \mathrm{~s}$. An additional input has been added which allows the user to set the duration of the noise event and, from there, the corresponding SEL is output. This level is calculated based on the receiver sound pressure level, $L_{e q}$, and Equation (1.7). Assuming the run-up noise event is constant, Equation (1.7) can be simplified to:

$$
S E L=10 \log \left(\left(t_{2}-t_{1}\right) 10^{L_{e q} / 10}\right) .
$$

This level is then used in the ANSI standard formula described in Chapter 1:

$$
\% \text { Awakenings }=-7.02+0.14 \mathrm{SEL} \text {, }
$$

and the percentage of people awoken is output.

These indices allow the user to calculate the extent to which people in communities will be awoken as a result of an engine run-up. If these values suggest that noise levels are excessive, a decision to relocate the aircraft, to use a different power setting; or to postpone to a later time can be made.

The next chapter will evaluate the modifications made to the GFPE code. Comparison of GFPE predictions with measurements and predictions from literature will be made and discussed. 


\section{Chapter 4}

\section{GFPE Model Evaluation}

There are many papers published in the literature that describe results from the measurement and prediction of sound propagation outdoors. In order to evaluate the modifications made to the GFPE model in Chapter 3, predictions were compared with data from the literature. Several literature sources are used in this chapter to compare and evaluate different aspects of the current GFPE model, including homogeneous and refracting atmospheres, mixed ground impedance, and the addition of turbulence.

\subsection{Homogeneous Atmosphere (No Refraction)}

Attenborough et al. compiled a set of benchmark cases for which the transmission loss over a finite impedance ground at 10,100, and $1000 \mathrm{~Hz}$ was determined for homogeneous, downward refracting, and upward refracting atmospheres [15]. This source has been widely used for outdoor sound propagation comparisons and is considered a highly reliable standard. Data from this benchmark paper was compared with GFPE predictions to ensure step size modifications were chosen to produce accurate and computationally efficient results. Figure 4.1 shows the published benchmark results for a homogeneous atmosphere, and Figure 4.2 shows the results from the GFPE prediction.

For each frequency, very good agreement - usually within $\sim 2 \mathrm{~dB}$ - was found. Interference effects close to the source were difficult to capture in some cases due to the step size, but the levels - particularly at $\sim 2 \mathrm{~km}$, a typical source-receiver distance in this work

- were extremely close, typically within 1-2 dB. 


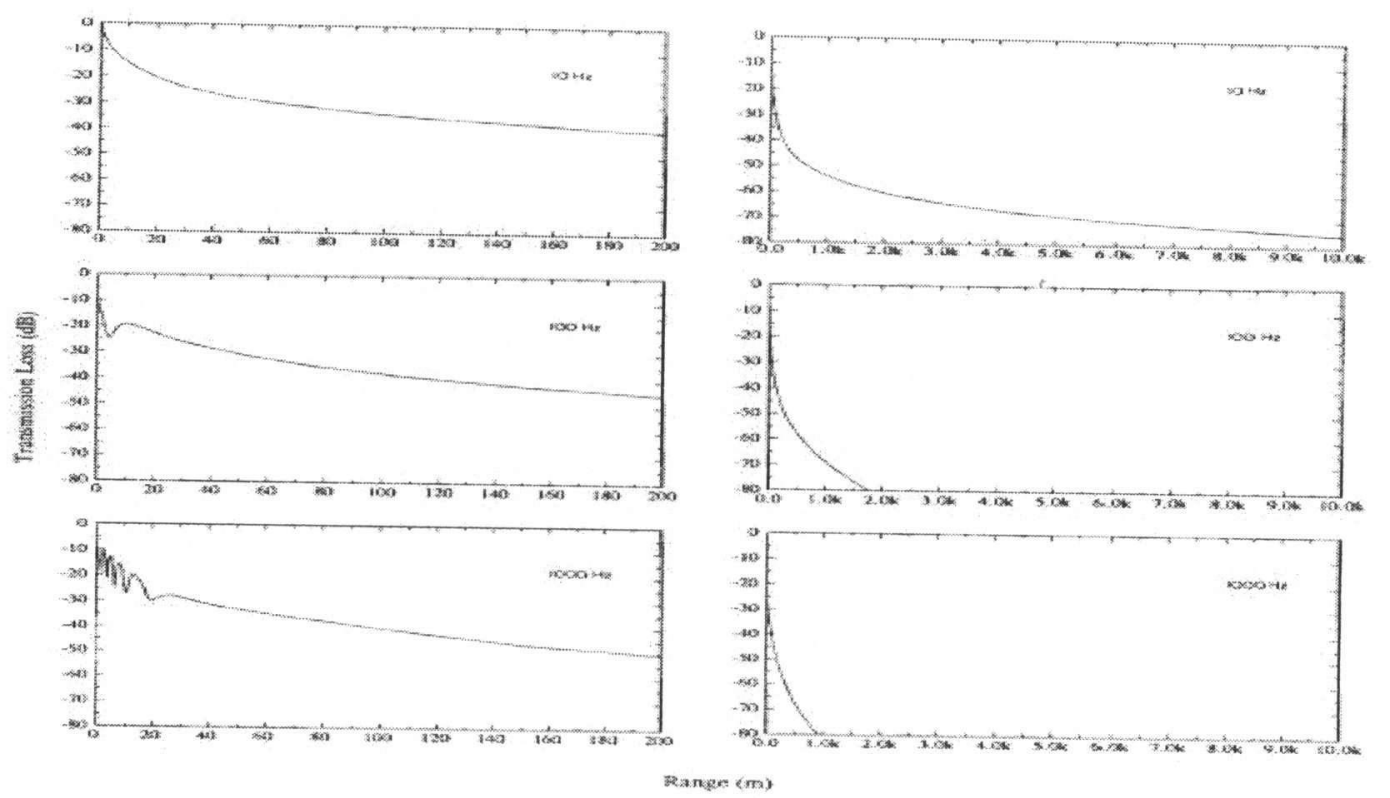

Figure 4.1: Transmission loss for the homogeneous atmosphere benchmark case. Input parameters include $h_{\text {source }}=5 \mathrm{~m}, h_{\text {receiver }}=1 \mathrm{~m}$, and normalized ground impedance for 10 , 100, and $1000 \mathrm{~Hz}$ of (38.79,38.41), (12.81,11.62), and (5.96, 2.46), respectively [15].
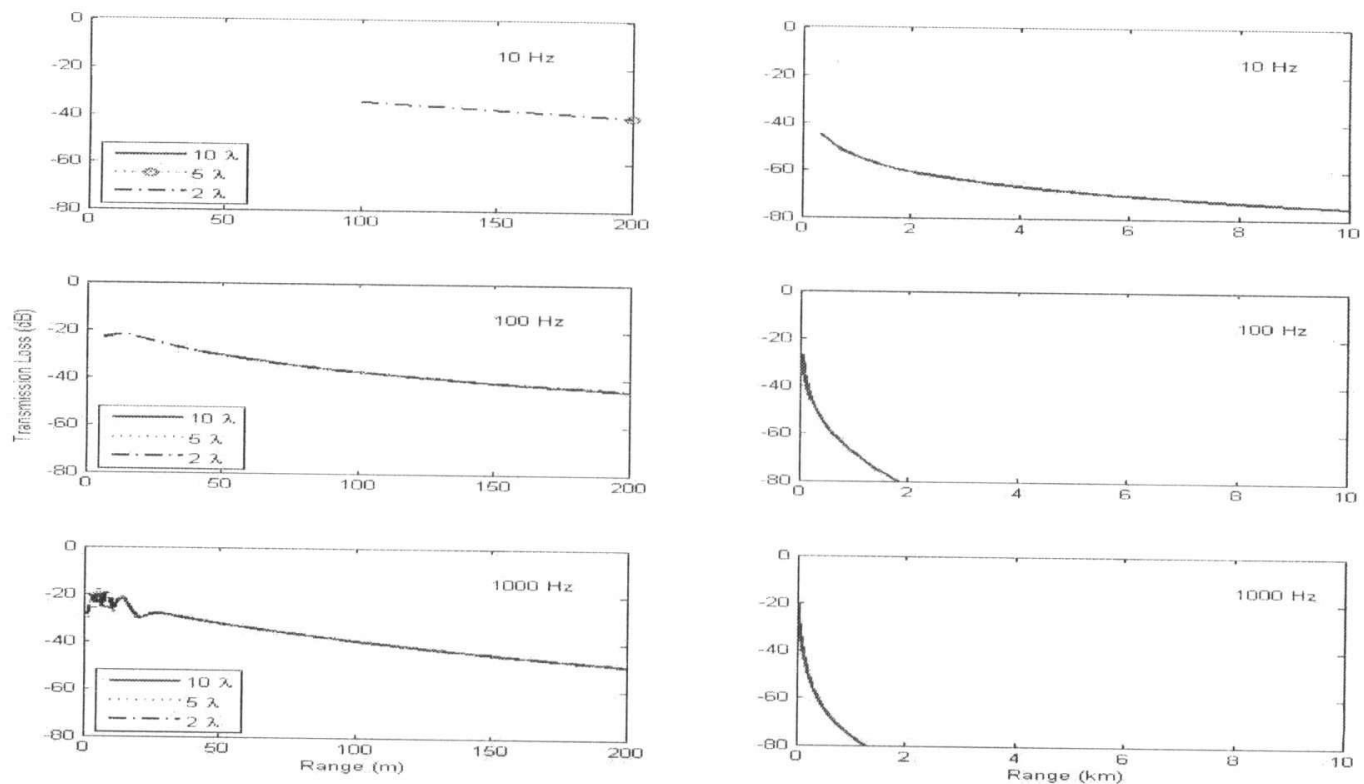

Figure 4.2: GFPE prediction corresponding to Figure 4.1. Parameters used in prediction include: FFT length $=16384$, vertical step size $=\lambda / 5$, vertical height $=250 \mathrm{~m}$, speed of sound $=343 \mathrm{~m} / \mathrm{s}$, surface wave integration number 250 . 


\subsection{Downward and Upward Refracting Atmospheres}

The previous case is very idealized - the atmosphere is not homogeneous: wind and temperature gradients cause the speed of sound to change with position and height, resulting in refraction of sound. Simple upward and downward refraction is considered here, again with the reference levels from the benchmark paper [15]. Figure 4.3 shows the transmission loss over $10 \mathrm{~km}$ in a downward refracting atmosphere from the benchmark paper; Figure 4.4 shows the corresponding GFPE prediction. Figures 4.5 and 4.6 show the transmission loss for the upward refraction case from both the benchmark case and the GFPE prediction, respectively.

For both refracting cases, very good agreement was obtained for both distances shown in Figures 4.4 and 4.6: benchmark and prediction values were within a couple of $\mathrm{dB}$ of each other. Although finer detail is at times missing due to the step size, the prediction curves' overall shapes look very similar to the benchmark curves' shape.

One major issue - not so much with the benchmark cases used for these comparisons, but with others - was step size. When choosing horizontal step sizes, too large a step will step right over more detailed ground features. This is particularly problematic at lower frequencies, since step sizes are fixed at a multiple of the wavelength. For instance, $10 \lambda$ at $25 \mathrm{~Hz}$ is $136 \mathrm{~m}$ : changes in ground type less than $136 \mathrm{~m}$ will not be modeled correctly for this step size. On the other hand, a step size that is too small generates numerical errors [56]. The generally acceptable horizontal step size range required to ensure accuracy with the GFPE model is from $\sim 5-10 \lambda$ to $\sim 50-100 \lambda[38,56]$. Since mixed ground will eventually be included, and may require smaller steps to represent the changes in ground impedance, $10 \lambda$ has been chosen for consistency.

From these first tests, it is apparent that the GFPE produced acceptable predictions for the transmission loss of single frequency, homogeneous ground impedance sound propagation in non-, upward-, and downward-refracting atmospheres, within $\sim 2 \mathrm{~dB}$ of accepted standard predictions. 

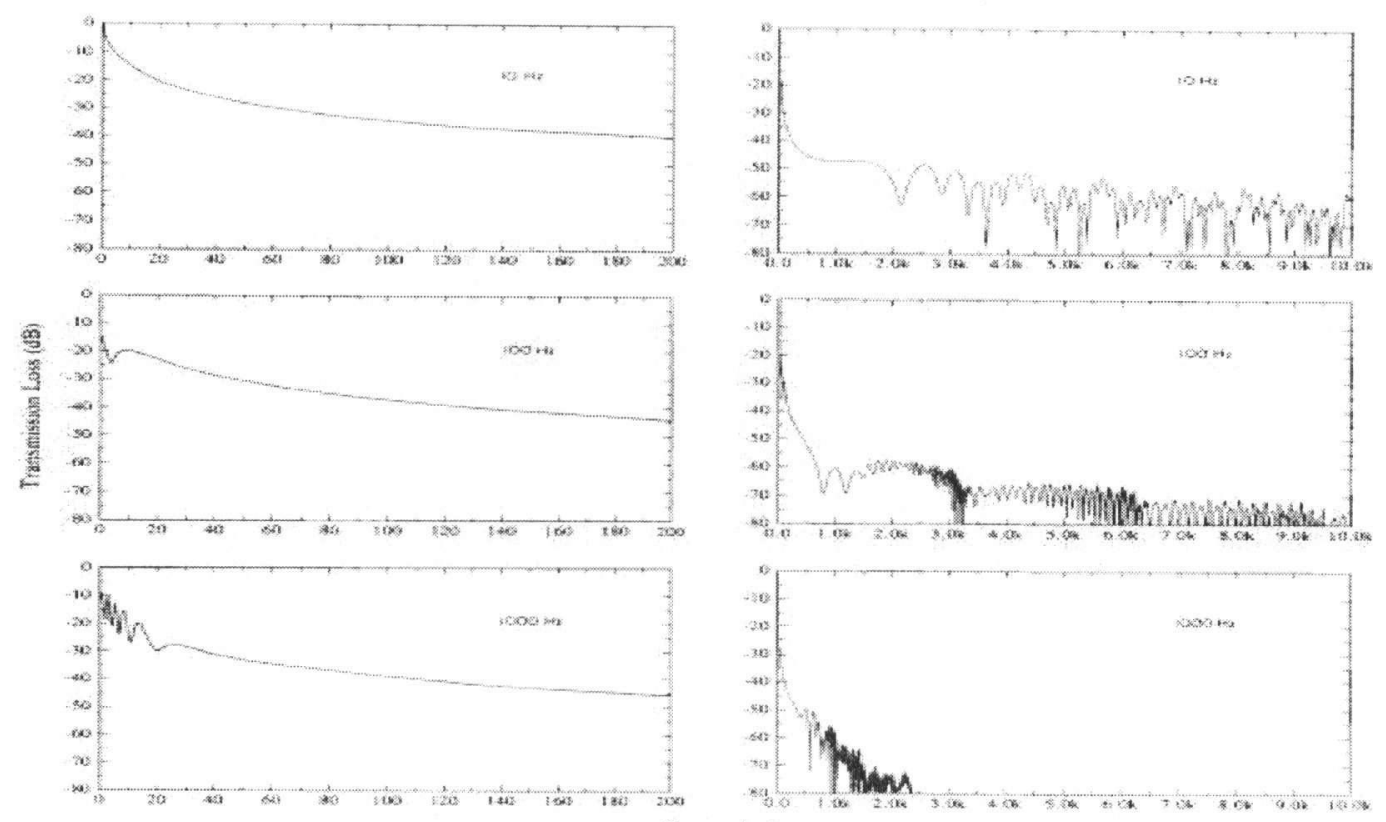

Figure 4.3: Transmission loss for the downward refracting atmosphere from benchmark paper. Similar input parameters as Figure 4.1, but with sound speed gradient of $+0.1 s^{-1}$ [15].
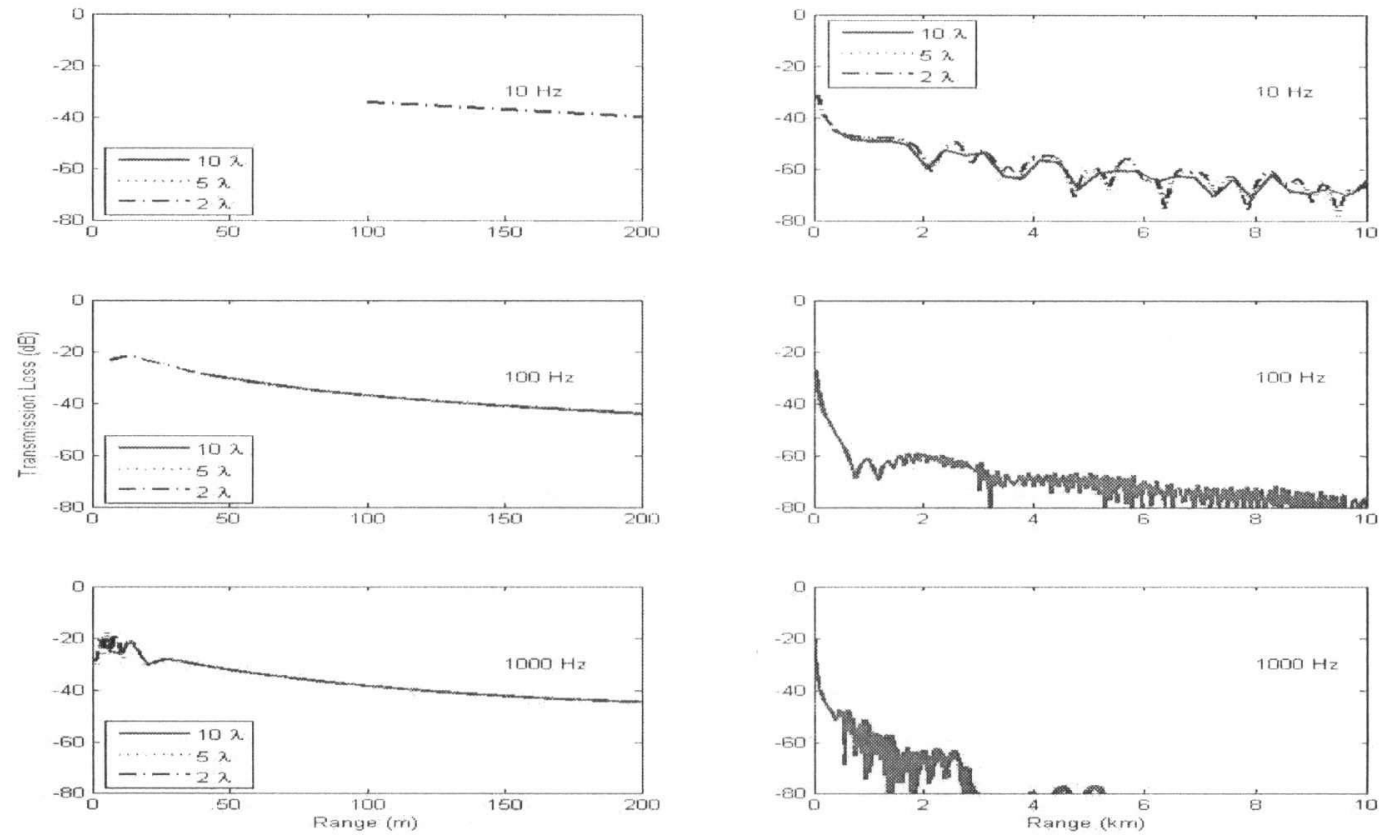

Figure 4.4: GFPE prediction corresponding to Figure 4.3. Similar input parameters as Figure 4.2, but with sound speed gradient of $+0.1 \mathrm{~s}^{-1}$. 


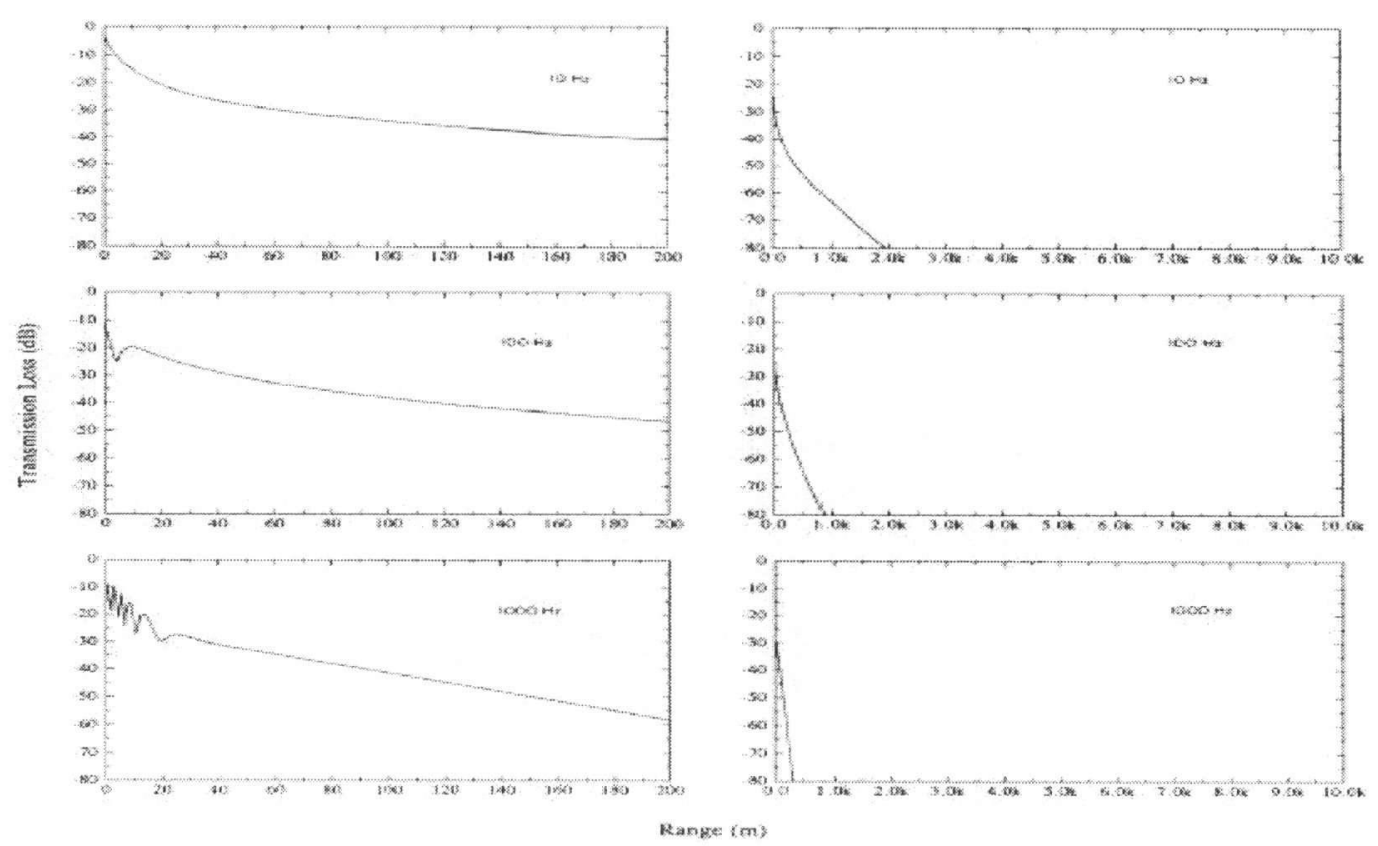

Figure 4.5: Transmission loss for the upward refracting atmosphere benchmark case. Similar input parameters as Figure 4.1, but with sound speed gradient of -0.1 $\mathrm{s}^{-1}$ [15].
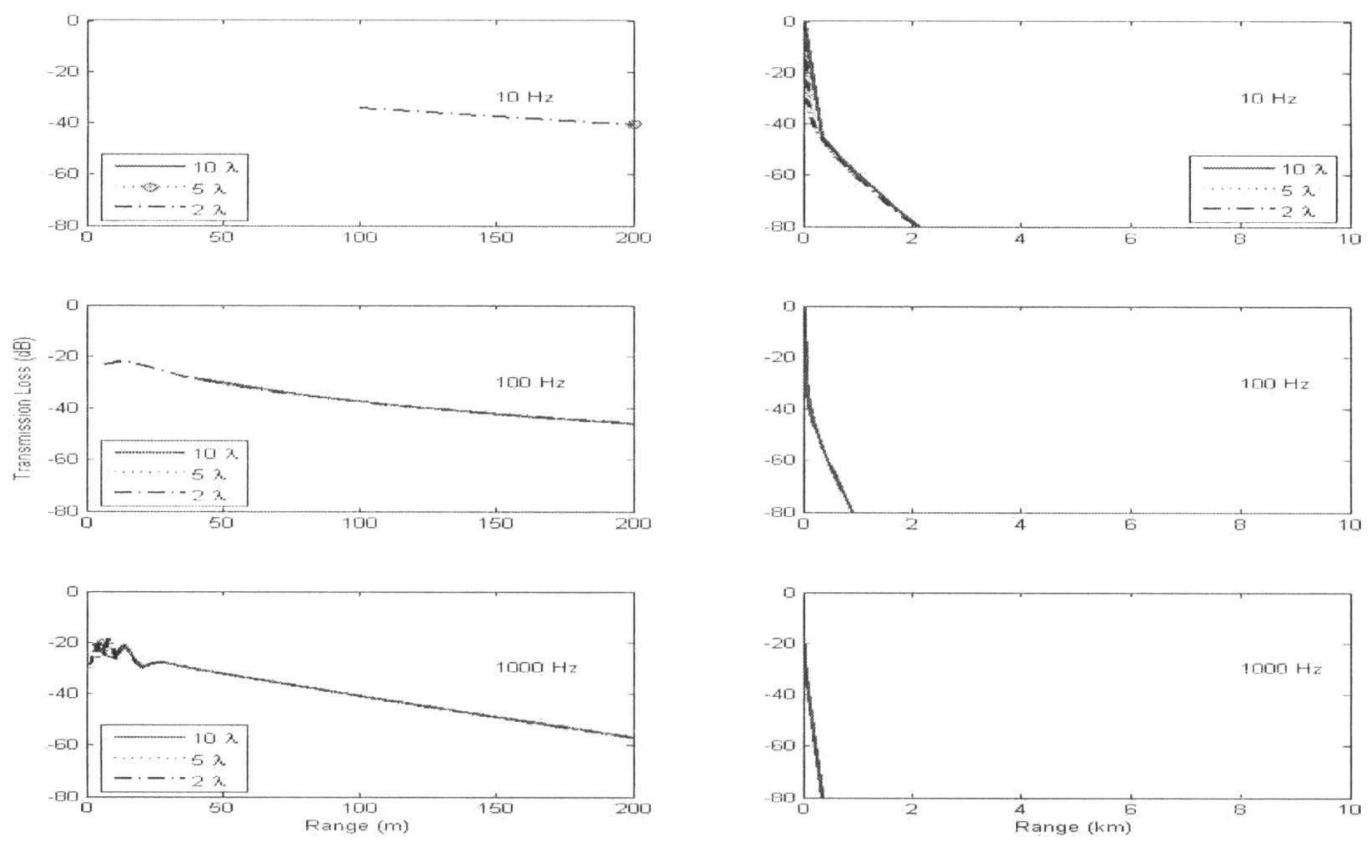

Figure 4.6: GFPE prediction corresponding to Figure 4.5. Similar input parameters as Figure 4.2, but with sound speed gradient of $-0.1 \mathrm{~s}^{-1}$. 


\subsection{Mixed Ground Impedance}

Unfortunately, much of work involving mixed ground impedance has only been for short distances, and often only for lower frequencies, conditions for which the existing GFPE model often cannot take a sufficient number of steps for predictions. In this section, comparisons are made with several different works, to ensure the GFPE modifications still produce accurate predictions. Two things are being tested in this section:

- impedance discontinuities; and

- the loop calculating multiple frequencies per simulation.

Both transmission loss and excess attenuation can be expressed as functions of distance for a single frequency, or as a function of frequency at one specific receiver location. For instance, in the benchmark paper [15], TL was expressed as a function of distance at a given frequency $(10,100$, or $1000 \mathrm{~Hz})$. Excess attenuation, as a function of both distance and frequency, is used in this section to compare sound levels published in literature with GFPE predictions.

In [16], a split-step Padé prediction model is compared with CNPE results. The spitstep Padé method is a parabolic model that solves for the operator $H$ using a second-order Padé expansion, rather than the spectral theorem discussed earlier [16]. The Padé expansion is a rational function, with polynomial expressions on the numerator and denominator. Its function is similar to a Taylor series expansion. The excess attenuation for upwind (upward refracting) and downwind (downward refracting) atmospheric conditions were predicted first; good agreement was found.

One prediction, shown in Figure 4.7, was available for a homogenous atmosphere over an impedance discontinuity [16]. Regrettably, this prediction was only done for one, lower frequency $(160 \mathrm{~Hz})$, and over a shorter source-receiver distance $(100 \mathrm{~m})$. Due to the horizontal step-size restriction (10ג), GFPE levels could only be calculated for four points. They have been plotted in Figure 4.8. Predictions for the source-receiver distance being hard only, as in the first $50 \mathrm{~m}$, and soft only, as in the second $50 \mathrm{~m}$ of Figure 4.7, were included for comparison in Figure 4.8. An estimate of the CNPE results from Figure 4.7 has also been plotted in Figure 4.8, showing very good agreement: all points are within 1 $\mathrm{dB}$, except the second, which is at the transition point. It is expected that, if more points were predicted, better agreement over the impedance discontinuity would be seen.

To ensure GFPE model predictions were accurate at all frequencies, and over all dis- 


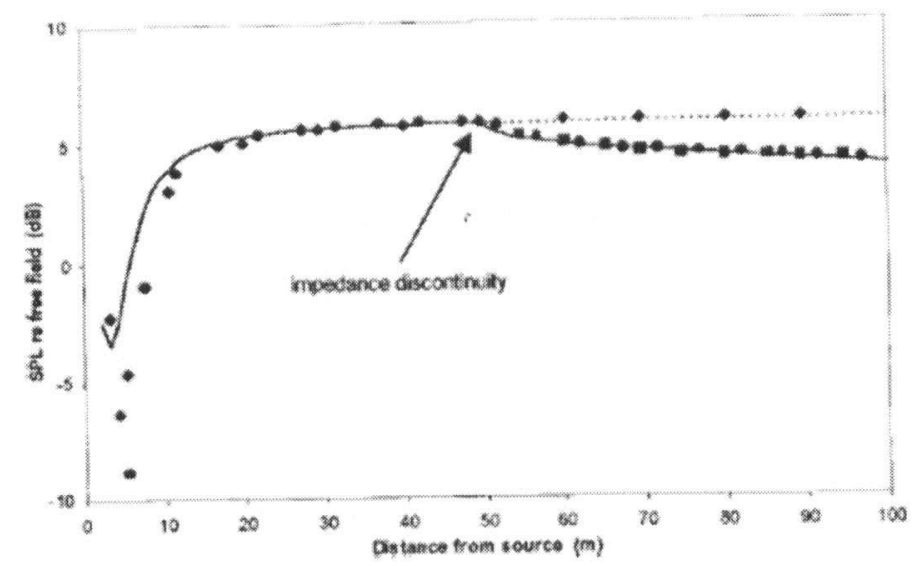

Figure 4.7: Excess attenuation for a mixed impedance ground. The solid line is for the CNPE results, the dots for the Padé predictions, the diamonds are for the single impedance case (160 HZ) [16].

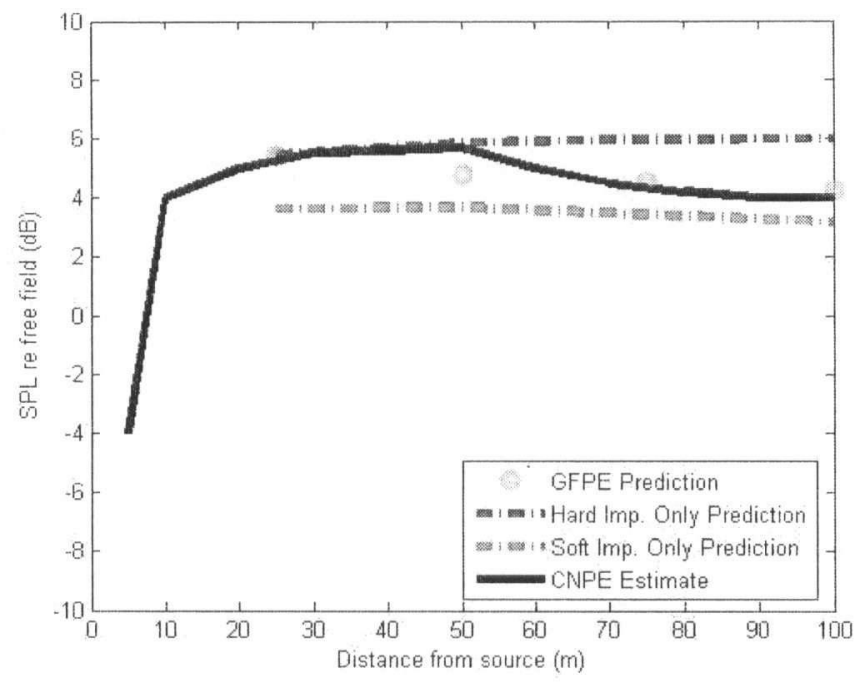

Figure 4.8: GFPE prediction corresponding to Figure 4.7. Parameters used in prediction include: $h_{\text {source }}=1.5 \mathrm{~m}, \mathrm{FFT}$ length $=16384, h_{\text {receiver }}=1.8 \mathrm{~m}, \Delta z=\lambda / 10, h_{\text {vertical }}=100$ $\mathrm{m}, \mathrm{c}=340 \mathrm{~m} / \mathrm{s}$, Delany and Bazley model $\sigma_{1}=2 \cdot 10^{5} \mathrm{kPa}, \sigma_{2}=2 \cdot 10^{2} \mathrm{kPa}$ ), discontinuity at $50 \mathrm{~m}$; surface wave integration number $=100 .(\bigcirc)$ is the GFPE prediction, (......) is the hard impedance only prediction, and (......) is the soft impedance only prediction, and (___) is the CNPE estimate from Figure 4.\%. 


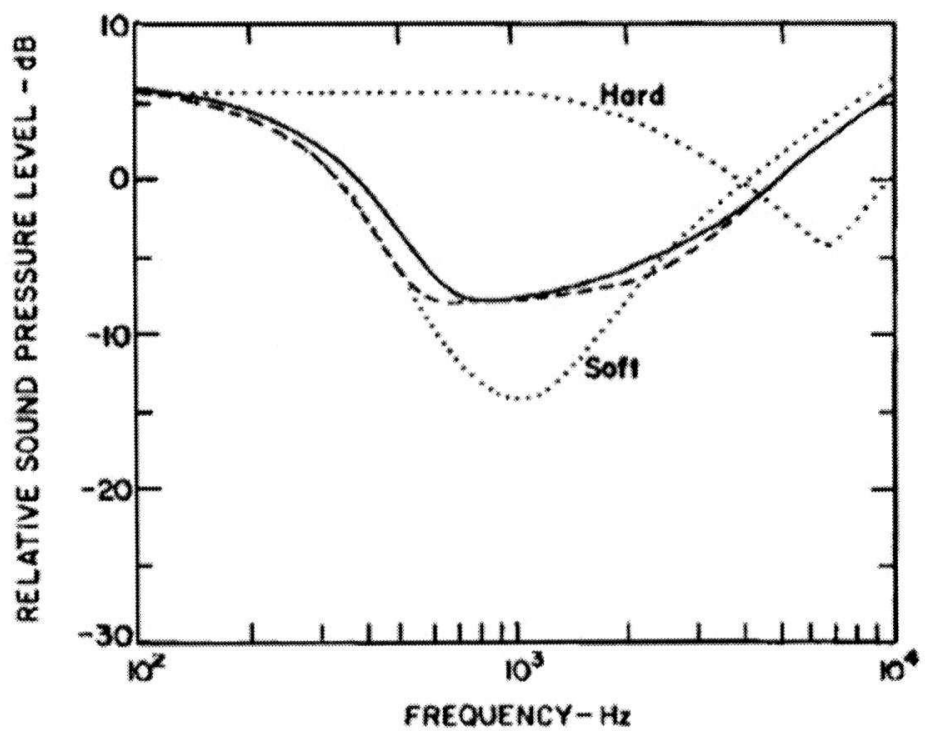

Figure 4.9: Comparison of calculated curves for two models across an impedance discontinuity: Rasmussen (____ ) and De Jong (_ _ _.). The dotted curves are predictions for all hard or all soft ground (no discontinuity). $h_{\text {source }}=1.5 \mathrm{~m}, h_{\text {receiver }}=1.5 \mathrm{~m}$, source-receiver distance $=5 \mathrm{~m}$, first portion of ground hard ( $\sigma_{1}=30000 \mathrm{cgs}$ rayls), second portion grass $\left(\sigma_{2}=300 \mathrm{cgs}\right.$ rayls $)[17]$.

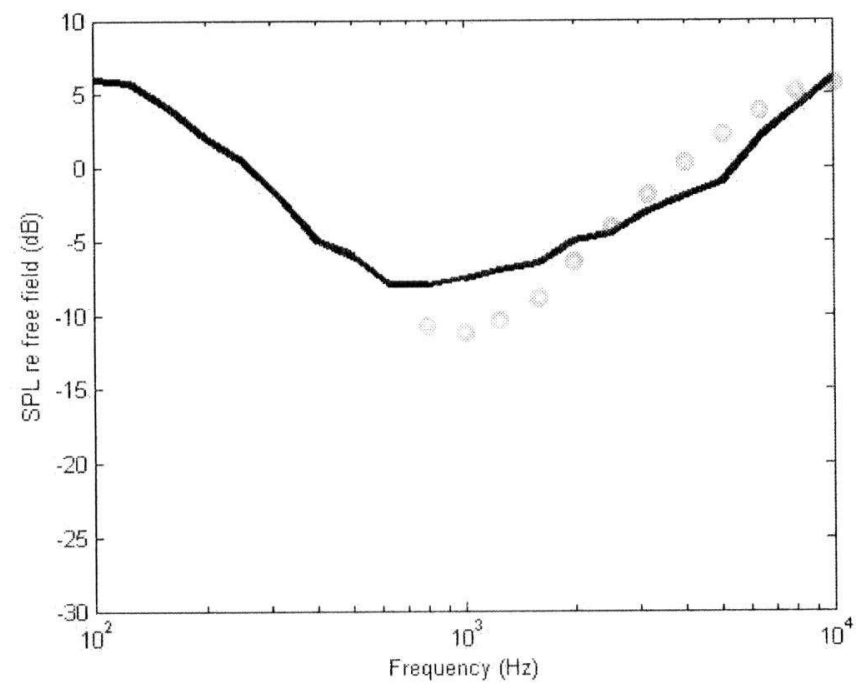

Figure 4.10: GFPE prediction corresponding to Figure 4.9. (O) is for GFPE predictions, and (_-_-) is an estimate of Ramussen's model from Figure 4.9 for comparison. 
tance ranges, predictions were also compared with predicted data from Daigle et al. [17], as illustrated in Figure 4.9. The corresponding prediction has been plotted in Figure 4.10. The Fourier transform number limited the frequency range at high frequencies. It is related to the number of vertical steps and thus is a function of $\lambda$; as $\lambda$ decreases, the number of steps for the fixed atmosphere height increases. For a given FFT length, there is a critical frequency beyond which the GFPE can no longer calculate sound pressure levels. Higher FFT lengths can be used, but the corresponding computational time increases substantially.

Low frequencies were restricted by the horizontal step size. In this case, since the impedance discontinuity occurs $5 \mathrm{~m}$ from the source, only frequencies with $\lambda \leq \frac{5 \mathrm{~m}}{10}$ (because it is stepping with $\Delta r=10 \lambda$ ) will accurately represent the geometry of the problem. Recall that this step size was chosen to optimize the trade-off between ground detail and larger step size. At any rate, these mixed-ground impedance predictions were within a few $\mathrm{dB}$ of the published values, and good agreement was found for mid- and high-frequencies.

Results for several other cases were available $[17,62]$, but could not be used in this work due to incompatibilities in the geometry (e.g., source height too close to ground, sourcereceiver distance too short, etc.).

\subsection{Turbulence}

In order to evaluate the GFPE model in the case of atmospheric turbulence, three phase screens, at two frequencies, were generated according to the parameters given in [9], to evaluate how well turbulent predictions compared with measured data. Measurements from [63] were 30-s octave band averages; single frequencies used here and in [9] were calculated as the square root of the product of the upper and lower limits of the octave band frequencies.

Weak and strong upwind refraction were compared in both [9] and [63], and both papers found that frequency had little effect on the variation of sound due to turbulence. The strength of the refraction - how quickly the speed of sound decreases with height in this case - seemed to determine how quickly the excess attenuation decreased to the steady shadow-zone level. These results are shown in Figures 4.11 and 4.12 for 424 and $848 \mathrm{~Hz}$, respectively $[9,63]$.

The predictions corresponding to these two figures are shown in Figure 4.13. The 
CROSSWIND PROPAGATTON (WEAK UPWARD REFRACTION)
UPWIND PROPAGATION (STRONG UPWARD REFR ACTION)

FREQUENCY $=424 \mathrm{~Hz}$
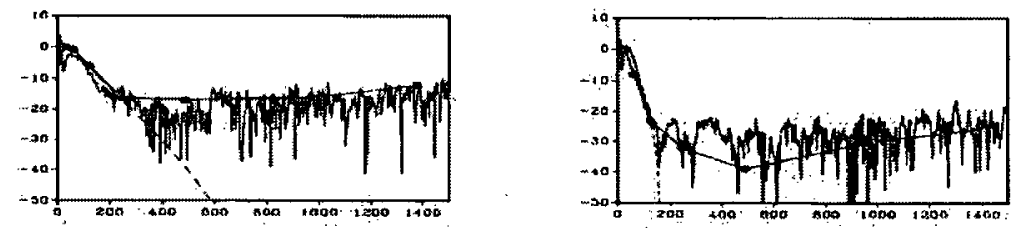

IRIAL 1
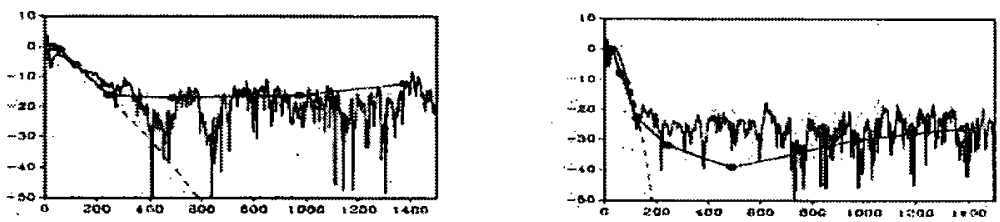

TRIAL 2

Figure 4.11: Relative sound pressure level prediction for two turbulence simulations using the CNPE at 424 Hz. Joint dots are Weiner et al.'s measurements; lines are Gilbert et al.'s CNPE predictions. The root-mean-square fluctuation and correlation length are 1.42 $\times 10^{-3}$ and $1.1 \mathrm{~m}$, respectively, $a=-0.5 \mathrm{~m} / \mathrm{s}$ and $-2.0 \mathrm{~m} / \mathrm{s}$ for weak and strong refraction, respectively $\left(c(z)=c_{0}=a \ln z / d\right), d=6 \times 10^{-3} \mathrm{~m}, Z=8.00+i 9.24$, source height $=$ $1.8 \mathrm{~m}$, receiver height $=1.5 \mathrm{~m}[9]$.

FREQUENCY $=848 \mathrm{~Hz}$
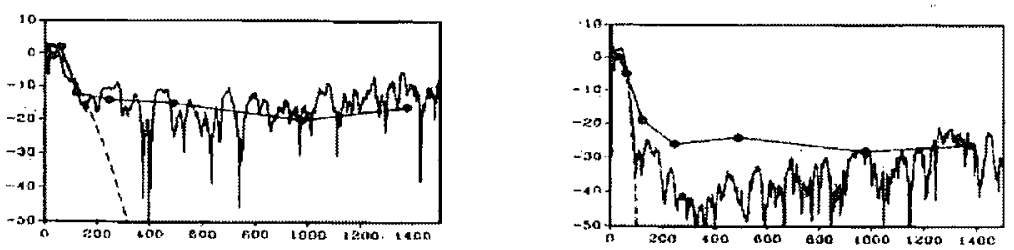

TRIAL 1
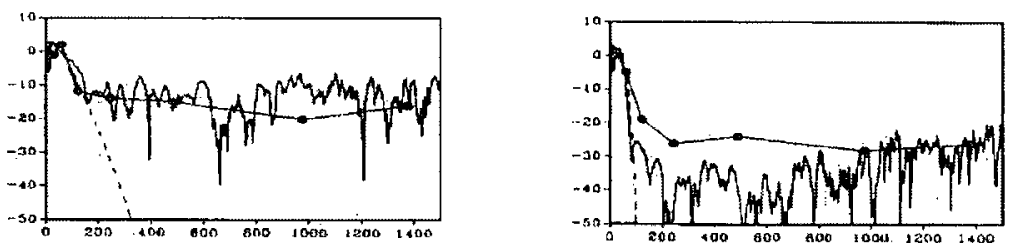

TRIAL 2

HORIZONTAL RANGE (In)

Figure 4.12: Relative sound pressure level prediction for two turbulence simulations using the CNPE at $848 \mathrm{~Hz}$. Joint dots are Weiner et al.'s measurements; lines are Gilbert et al.'s CNPE predictions. Same parameters as Figure 4.11, except $Z=5.17+i 5.57$ [9]. 

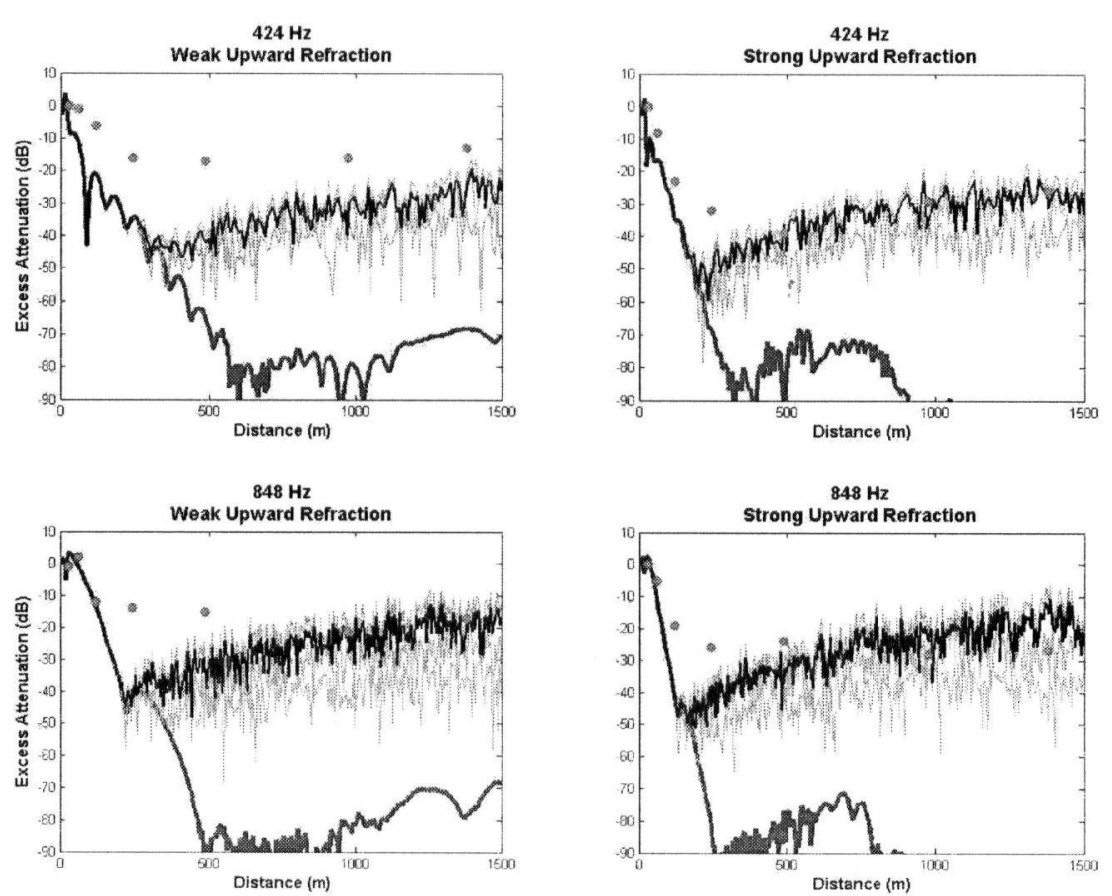

Figure 4.13: GFPE predictions with turbulence in a weak and strong upward refracting atmosphere. Parameters correspond to Figures 4.11 and 4.12. (_-) is the non-turbulent prediction, ( ), ( ), and ( ) correspond to the three predictions with turbulence, (_-_) is the $d B$ average of the three turbulent predictions, and (•) are Weiner et al.'s measurements from Figures 4.11 and 4.12.

faster rate at which the sound decreases to the shadow-zone region level in the strong upward refraction case is apparent in these predictions. The logarithmic average of the three phase-screen predictions matches up well with the measured results from [63] in all cases, except for weak refraction at low frequency. Predictions using turbulence are much closer to measured levels than predictions without turbulence. It is hypothesized that averaging predictions from additional phase screens could produce superior results; however the generation of phase screens (particularly at higher frequencies), as well as the multiple predictions, become extremely long computationally.

A discussion with Di [38] suggested that turbulence is only significant in upward refracting atmospheres. To verify whether this is supported by prediction, the same three phase screens were applied to a homogeneous, a strong downward refracting, and a weak downward refracting atmosphere. Figure 4.14 shows these GFPE predictions. 

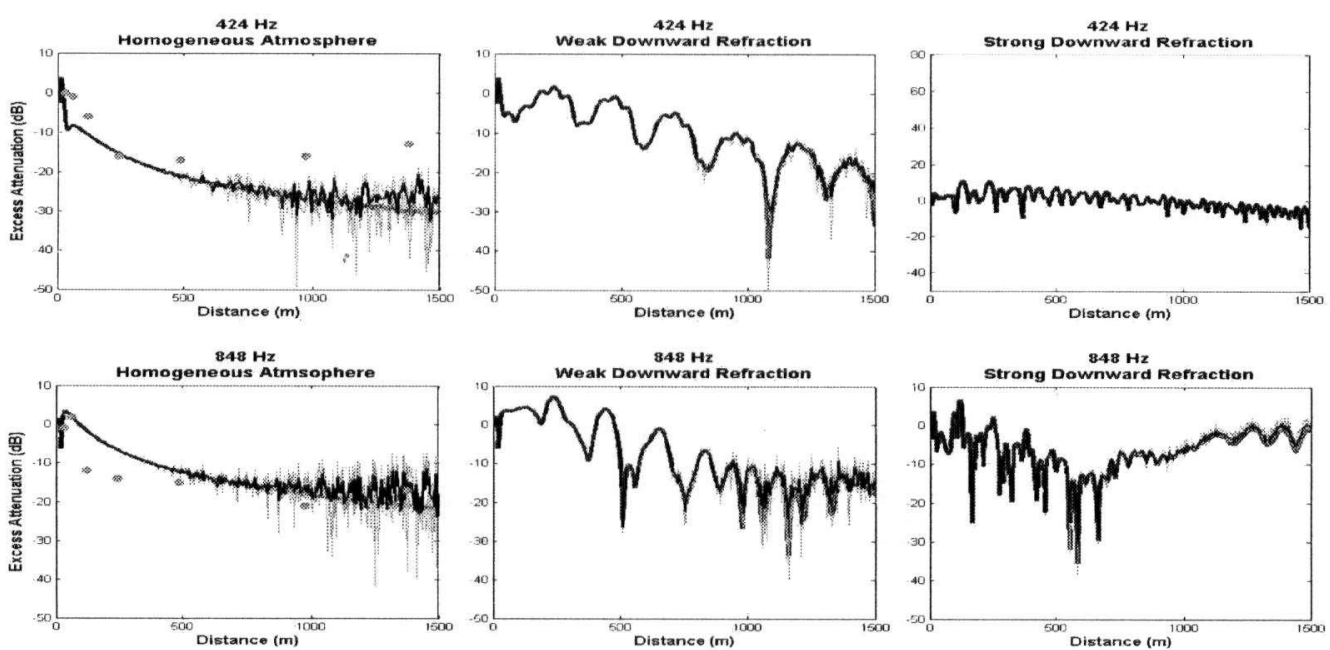

Figure 4.14: GFPE predictions with turbulence in a homogeneous (no refraction), weak, and strong downward refracting atmosphere. All other parameters are the same as Figures 4.11 and 4.12. ( ( ) is the non-turbulent prediction, ( ) ), ( ), and ( ) correspond to the three predictions with turbulence, (_-_) is the $d B$ average of the three turbulent predictions, and $(\bullet)$ are Weiner et al.'s measurements from Figures 4.11 and 4.12 for the weak upward refracting atmosphere.

The strength of refraction, or the change in sound speed with height, was estimated by Gilbert et al. in [9] for Weiner et al.'s data [63]. Although Gilbert et al.'s predictions did work well (see Figures 4.11 and 4.12), one possible explanation for the poor $424 \mathrm{~Hz}$ weakupward refracting results, other than perhaps the need for averaging more simulations, is that the $424 \mathrm{~Hz}$ weak refraction measurements taken by Weiner et al. were in fact weaker than Gilbert et al. predicted. Plotting Weiner et al.'s measurements on the homogeneous atmosphere graphs obtained from GFPE predictions for comparison, the homogeneous case is no closer (note that the scales are different: the vertical axis extends lower in Figure 4.13 to show the non-turbulent prediction).

Another possible explanation has to do with the frequency: Weiner et al.'s measurements were for the band of frequencies from $300-600 \mathrm{~Hz}$, not the single frequency, $424 \mathrm{~Hz}$. Perhaps certain frequencies within that range were diffracted into the shadow zone more than at $424 \mathrm{~Hz}$, resulting in a higher band average. It should be remembered that a major assumption was made at the beginning of this work - that broadband sound can be approx- 
imated by the third-octave band central frequencies.

It is thus not completely clear why the $424 \mathrm{~Hz}$ weak upward refraction predictions are so different from the measurements. These turbulence predictions do, however, calculate sound pressure levels closer to measurement than for the equivalent non-turbulent cases.

As expected, with the homogeneous and, particularly, with the downward refraction predictions, not much change was observed with the inclusion of turbulence (in fact, the dark blue $\mathrm{dB}$ average curve covers much of the turbulence curves). These results suggest that, under inversion conditions (temperature, and thus the speed of sound, increase with height), turbulence need not be incorporated in the GFPE model for accurate predictions.

\subsection{Summary}

In homogeneous, refracting; mixed-impedance, and turbulent atmospheres, the GFPE model, with all of its modifications, predicted the transmission loss and the excess attenuation very well. It must be remembered that accurate inputs are crucial for accurate outputs - sound speed profiles were estimated for refraction, ground impedance was calculated from models (which, as has already been shown, have a fair bit of uncertainty themselves), single frequencies approximated octave (or other) bands, and larger step sizes approximated steps that were fractions of a wavelength. With all this in mind, it can be concluded that predicted sound levels are sufficiently accurate - usually within $\sim 2 \mathrm{~dB}$ of expected levels - to allow their application in the present work.

It should be noted that while most combinations of the different types of ground and atmospheres were considered, some were not, for the simple reason that no good examples could be found in the literature. It would have been particularly informative to find results in the literature for mixed ground impedance and turbulence. Unfortunately, the only thing found regarding these two factors was a statement that predictions and measurements for such a combination do not exist in the literature [16]! The following section will present predictions involving measured atmospheric conditions, and compare them with measured noise levels in communities. 


\section{Chapter 5}

\section{Run-up Noise and Annoyance Predictions ${ }^{1}$}

With the successful results obtained evaluating the GFPE model, it is now possible to use it to predict noise levels, and their corresponding annoyance (sleep disturbance). Nighttime complaints associated with engine run-up noise were monitored from January 2005 until June 2006. A search for corresponding engine run-up events was conducted for each of these complaints in the YVRAA Incident Database. In cases where complaints and run-up events were correlated, noise data from the NMT(s) closest to the source of the complaint was saved for the period of time when the run-up took place.

The run-up events identified according to this procedure were selected for GFPE sound pressure level predictions in this chapter. Input parameters were entered with all available information; some cases required additional estimates (for example, the aircraft heading, which was sometimes missing from the reports), depending on what was recorded in the complaint and engine run-up incident reports. SEL, the acoustical quantity used to determine annoyance, was also calculated, based on the duration of the event, as indicated in the incident reports.

Two sources of uncertainty come into play at this point: uncertainty in the predictions by the model itself, and uncertainty resilting from the model's sensitivity to the input parameters. The previous chapter concluded that the model inherently predicted levels with a high level of accuracy, typically within $\sim 2 \mathrm{~dB}$. To understand the dependence on the input parameters, a simple case has been used as a reference, with which predictions for other cases are compared. Prediction input parameters were, one by one, varied slightly. There will always be some uncertainty associated with these input values, and while they must

\footnotetext{
${ }^{1} \mathrm{~A}$ version of this chapter will be submitted for publication. Scherebnyj, K. and Hodgson, M. Prediction of Noise Levels and Annoyance from Aircraft Run-ups at the Vancouver International Airport.
} 
always be chosen with as much diligence as possible, a concept of the variability associated with any assumptions made should be kept in mind when interpreting the final prediction results.

This chapter begins by explaining how the atmosphere was modeled. Uncertainties related to the input parameters are then discussed, followed by a sensitivity 'analysis of the GFPE model. Predictions of run-up events that occurred over the last year and a half are then assessed and summarized, along with their corresponding community annoyance (percentage of people awoken), as determined by SEL.

\subsection{Atmospheric Predictions}

There are three general states that exist for a static atmosphere: stable, unstable, and neutral. In a stable atmosphere, temperature increases with height. The shape of the temperature profile increase can reasonably be approximated as parabolic [64]. To approximate this profile, the temperature at $6 \mathrm{~m}$ was entered into the input file, and a parabola was fit to this value, along with points at $0 \mathrm{~m}$ and at $200 \mathrm{~m}$, which were $2^{\circ} \mathrm{C}$ lower and $6^{\circ} \mathrm{C}$ higher than the $6 \mathrm{~m}$ temperature, respectively. From the parabola coefficients, temperatures were calculated at several heights; these temperatures were then used to calculate the sound speed profile.

The wind profile power law,

$$
\frac{u(z)}{u(10)}=\left(\frac{z}{10}\right)^{1 / 7},
$$

where $u$ is wind speed and $z$ is height above the ground, was used to calculate the variation of wind speed with height. This is commonly used to approximate wind profiles in the absence of information other than the wind speed at a given height (in this case $10 \mathrm{~m}$ ) [64]. The wind vector was then projected onto the source-receiver direction and added to the sound speed. If the wind speed at $10 \mathrm{~m}$ is greater than $\sim 5 \mathrm{~m} / \mathrm{s}$, the positive gradient probably won't exist at higher elevations due to the larger amount of turbulent mixing, and the assumption that the state of the atmosphere is stable is no longer valid.

Two factors make this the most interesting of the three general atmosphere types: first, stable atmospheres can be predicted with relative certainty; and second, noise levels in downward refracting atmospheres tend to be higher, because the sound ducts along the 
ground. As higher noise levels are expected in this type of atmosphere, it is considered the 'worst case scenario' and therefore can be used as a default when other information regarding the state of the atmosphere is unknown.

An unstable atmosphere is one for which temperature decreases with height at a rate greater than $0.0098^{\circ} \mathrm{C} / \mathrm{m}$ (this is the dry adiabatic lapse rate of the atmosphere). Instability indicates that vertical movement of air packets is not restricted. The temperature profile in a neutral atmosphere decreases at a rate of $0.0098^{\circ} \mathrm{C} \mathrm{m}^{-1}$ - here vertical air movement is neither suppressed nor encouraged.

Temperatures in the first $10 \mathrm{~m}$ above the ground do not follow a simple curve. This part of the profile is almost impossible to know without making measurements at several heights. Although in unstable conditions temperature also decreases with height, nighttime unstable atmospheres are not common [64]. For these reasons, when the nighttime temperature decreased from the height of 1.6 to $6 \mathrm{~m}$, as long as the wind speed was below $5 \mathrm{~m} / \mathrm{s}$, neutral conditions were assumed. Similarly, if temperature increased between these two points, stable conditions were assumed. The wind profile power law was used to estimate the wind profile in all cases.

It is somewhat difficult to fully accept how the stable and neutral atmospheres were chosen, since the temperature change over that $4.6 \mathrm{~m}$ (from 1.6 to $6 \mathrm{~m}$ ) may not be representative of the full height above the ground through which sound propagates (e.g. up to $200+m$ ). Accurate prediction of temperature profiles close to the ground (within the lower $\sim 10 \mathrm{~m}$ ) is complicated by various effects, such as strong boundary layer interactions and molecular mixing right at the ground level.

Twenty-two run-up events with associated noise complaints, spaced over 16 nights, were identified according to the procedure described at the beginning of this chapter. Each runup event has been plotted in Figure 5.1, according to the change in temperature between 1.6 and $6 \mathrm{~m}$, and the wind speed at $10 \mathrm{~m}$ at the time of the run-up event. This indicates the conditions that were most commonly associated with run-up complaints, and how many events took place in stable or neutral atmospheres.

Although there is much scatter in Figure 5.1, the best-fit slope agrees with expectation: the smaller the change in temperature (i.e. the less stable it is), the stronger is the wind. This supports the earlier argument that the atmosphere will no longer be stable once the wind reaches a certain speed [64]; Figure 5.1 suggests that this cutoff may be closer to 4 


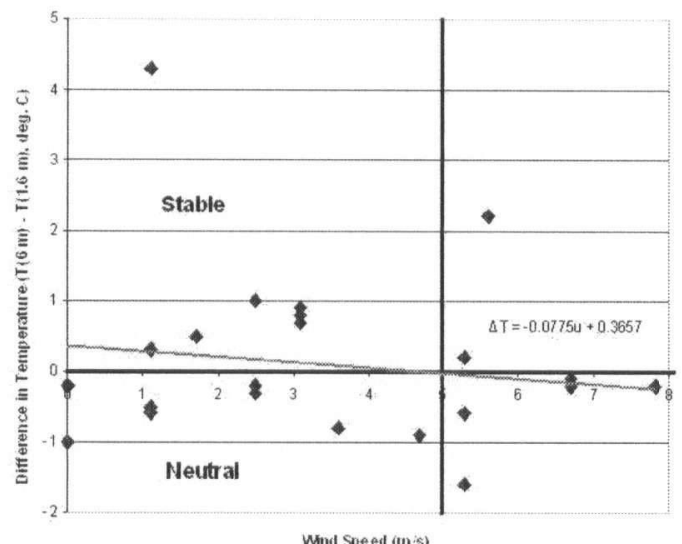

Figure 5.1: Atmospheric stability test: run-ups in the upper left hand corner took place under stable conditions; run-ups in the lower left hand corner took place under neutral conditions. Strong winds make it difficult to predict the atmospheric conditions on the right hand side.

$\mathrm{m} / \mathrm{s}$ than $5 \mathrm{~m} / \mathrm{s}$.

\subsection{Prediction Uncertainty}

There are many sources of uncertainty associated with predicting community disturbance from run-up noise. Aside from the uncertainties discussed earlier associated with issues such as the transmission loss through houses, uncertainty is also associated with factors such as the source (the aircraft radiation characteristics), the time and geometry (source and receiver positions, relative to one another) of the event, atmospheric assumptions, and complaint information.

Radiation characteristics are only available for the three aircraft measured for the three joint UBC/YVRAA projects: the Beechcraft 1900, the Dash-8, and the CRJ200. Since, however, run-ups at YVR are not limited to these three aircraft, for run-ups where other aircraft are being tested, one of these three measured aircraft has been 'substituted' in, to simulate the actual aircraft's levels. The substitutes (i.e. a Dash- $8^{2}$ for a D2 ${ }^{3}$, a CRJ200 for a $767^{4}$, etc.) may not always be appropriate. The orientation of the aircraft (for directivity)

\footnotetext{
${ }^{2}$ The Dash- 8 is a twin turboprop aircraft, designed to hold approximately 75 passengers.

${ }^{3}$ The D2, also known as the Beaver, is a single engine propeller bush plane.

${ }^{4}$ The Boeing 767 has two high-bypass engines, and can seat up to 375 passengers depending on the model.
} 
was not always known, and if it is, it is possible that the aircraft did not line up exactly as was recorded. In addition, the correct power setting of the aircraft is not always as indicated on the incident report, if the operator decided to test at other power settings.

The time requested for the run-up may not be the actual time at which the run-up took place; delays are not always noted. For the run-up noise measurement performed in this work, and previous run-up noise experiments, there is an uncertainty associated with the receiver positions and heights ( $1.4 \mathrm{~m}$ heights were approximate). This can be very important in terms of ground effect cancelation.

The assumptions involved when simplifying the atmosphere for GFPE model purposes are very difficult to make. A decision whether stable, neutral, or unstable conditions existed at the time of the run-up must be made, which in turn will help guide the user to choose the correct temperature and wind profiles. Furthermore, the only data available are averaged over hourly periods - this is not perfectly representative of the conditions at the exact time the event took place. Inherent uncertainties are also involved in the measurement equipment.

Complaint information, as discussed in Chapter 1, can be unreliable. For instance, it is difficult to ascertain that the caller correctly identified at what time the excessive noise levels took place (phone calls often come in the next day, with the caller making vague statements such as 'last night, between 2:30 and 5:30 AM").

Preflight run-ups interfere with run-up noise complaints: some propeller aircraft need to run their engines prior to flight; with flights leaving early in the morning, these runs are often performed around 4-7 AM. Aircraft operators do not need to request special permission for these run-ups, as they are part, of the regular flight procedure; however they are thought to be the source of many early morning complaints.

Unfortunate as the case may be, it is impossible to output correct values with incorrect inputs. In order to obtain an understanding of the sensitivity of each of the prediction model inputs, a reference case was compared with predictions for other cases, for which inputs were varied according to the magnitude of their expected uncertainty: The parameters for the reference case were as follows:

- source-receiver distance of $2 \mathrm{~km}$;

- one transition point mid-way, at $1 \mathrm{~km}$, dividing the first half, concrete (or any other acoustically hard surface), from dry, loamy grass; 


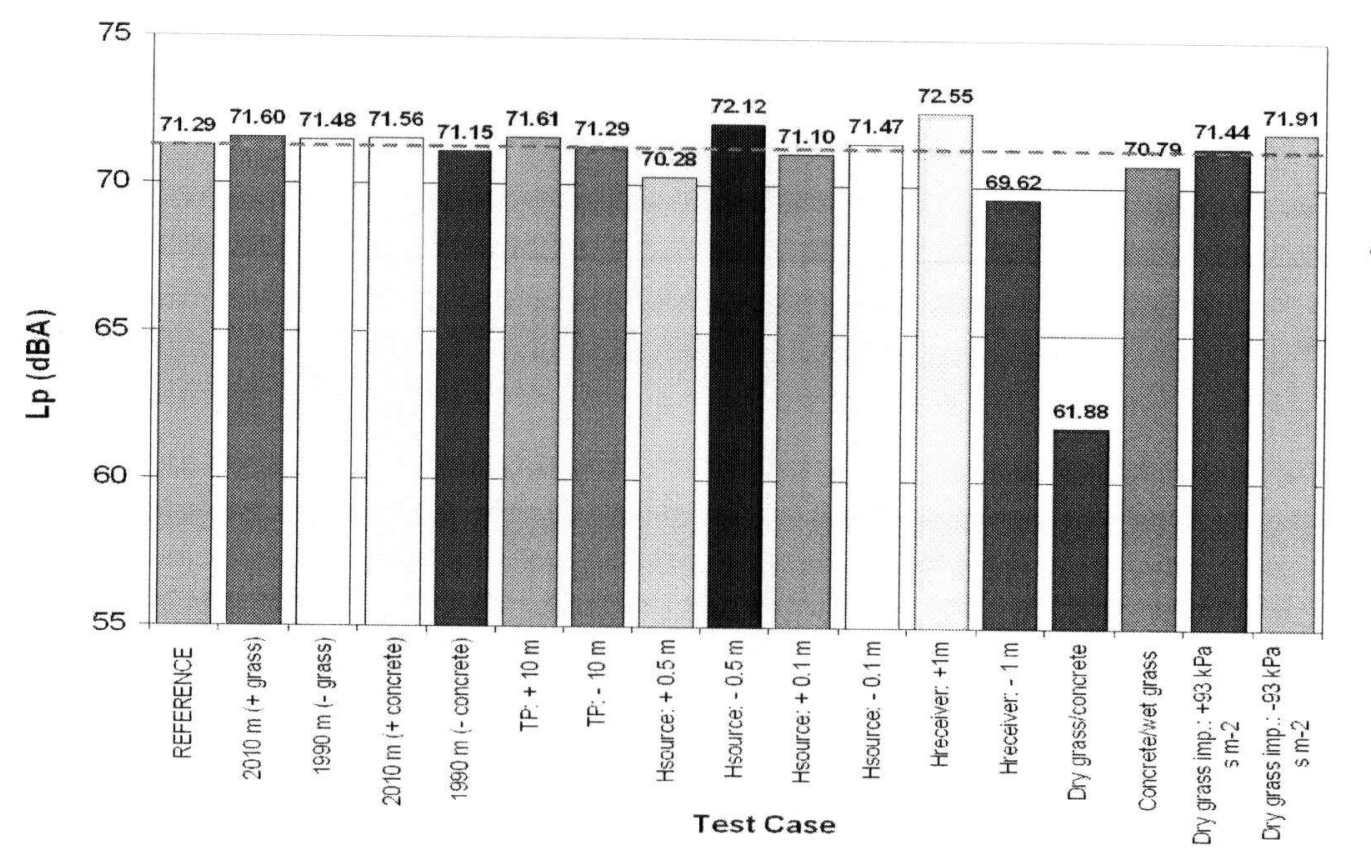

Figure 5.2: Reference test case compared with predictions for input variations in position (distance) and ground type. (___ ) is the reference level.

- source, Dash-8, at Position 5, running up at full power; source height $3 \mathrm{~m}$ above the ground;

- receiver height $6 \mathrm{~m}$ above the ground; and

- stable atmosphere, with no wind, with a temperature of $12^{\circ} \mathrm{C}$ at $6 \mathrm{~m}$.

A-weighted levels from $25 \mathrm{~Hz}-2 \mathrm{kHz}$ were predicted and compared; the frequency range from $25 \mathrm{~Hz}-2 \mathrm{kHz}$ was also extended, for comparison with the reference range. Only one change was considered at a time.

The first set of comparisons, relating to position and ground type, is shown in Figure 5.2. The first four cases relate to the total source-receiver distance: $10 \mathrm{~m}$ is added or subtracted to either the concrete or grass portion of the ground. The following two predictions shift the transition point between concrete and grass forward and backward $10 \mathrm{~m}$. The next six predictions are for source and receiver height variations. The last four have to do with the ground type and order: the first reverses the order of the concrete and grass, the second shows the change due to the grass being wet, and the last two are with the ground impedance calculated for a flow resistivity which is $93 \mathrm{kPa} \mathrm{s} \mathrm{m}^{-2}$ greater and smaller than 
that used in the reference case [1].

One very interesting result from Figure 5.2 is that, at a distance of $2 \mathrm{~km}$ - chosen as it was comparable to the distance between most run-up pad / NMT combinations - changes in distance, small changes in source/reciever heights, and variations in ground impedances have very little effect on A-weighted, $25 \mathrm{~Hz}-2 \mathrm{kHz}$ predicted sound pressure levels. Extending the frequency range three third-octave bands higher to $4 \mathrm{kHz}$ predicted $72.05 \mathrm{dBA}$ - again a very minor change. On the premise that a young, healthy-hearing person can barely distinguish a difference of $1 \mathrm{dBA}$, these variations are not significant.

The case for which predictions were the most different from the reference case was when the ground types were reversed: i.e. the first kilometer was grass, and the second concrete. Two conclusions can be drawn from this: first, the order in which the ground type is entered into the model makes a big difference, and second, when planning an airport, run-up noise can be decreased by surrounding the runways, taxiways, and run-up pads with grass, as is the case at YVR.

The last three results in Figure 5.2 are quite interesting. Only slight changes $(<1 \mathrm{dBA})$ in the overall sound pressure level were predicted when the grass portion of the ground was wet, and also when the flow resistivity used to calculate the impedance was varied. As long as information is available on distances, positions, and ground types, the error associated with the uncertainties in position are small in the final predicted levels.

Atmospheric effects were expected to influence the propagation of sound considerably, and they have, therefore, been considered separately. The first thirteen predictions in Figure 5.3 are related to the wind speed at $10 \mathrm{~m}$ : profiles were made according to the wind profile power law. Positive values are downwind, negative values are upwind. In either direction, predictions stray further from the reference the more the wind is increased. As expected from theory, propagation against the wind results in quiet areas. In real-world measurements, these levels will not be as low as shown in Figure 5.3, since turbulence scatters sound into the shadow zone. Sound propagation in the same direction as wind also leads to decreases in $L p$; however these are not as drastic as for upwind propagation. Wind is clearly a very important input parameter - incorrect assumptions about it can lead to predicted levels that are drastically different from the correct value.

Temperature trials in the next five cases list the temperatures at $6 \mathrm{~m}$ along with the state of the atmosphere. According to the temperature predictions, errors of $\pm 1^{\circ} \mathrm{C}$ in the 


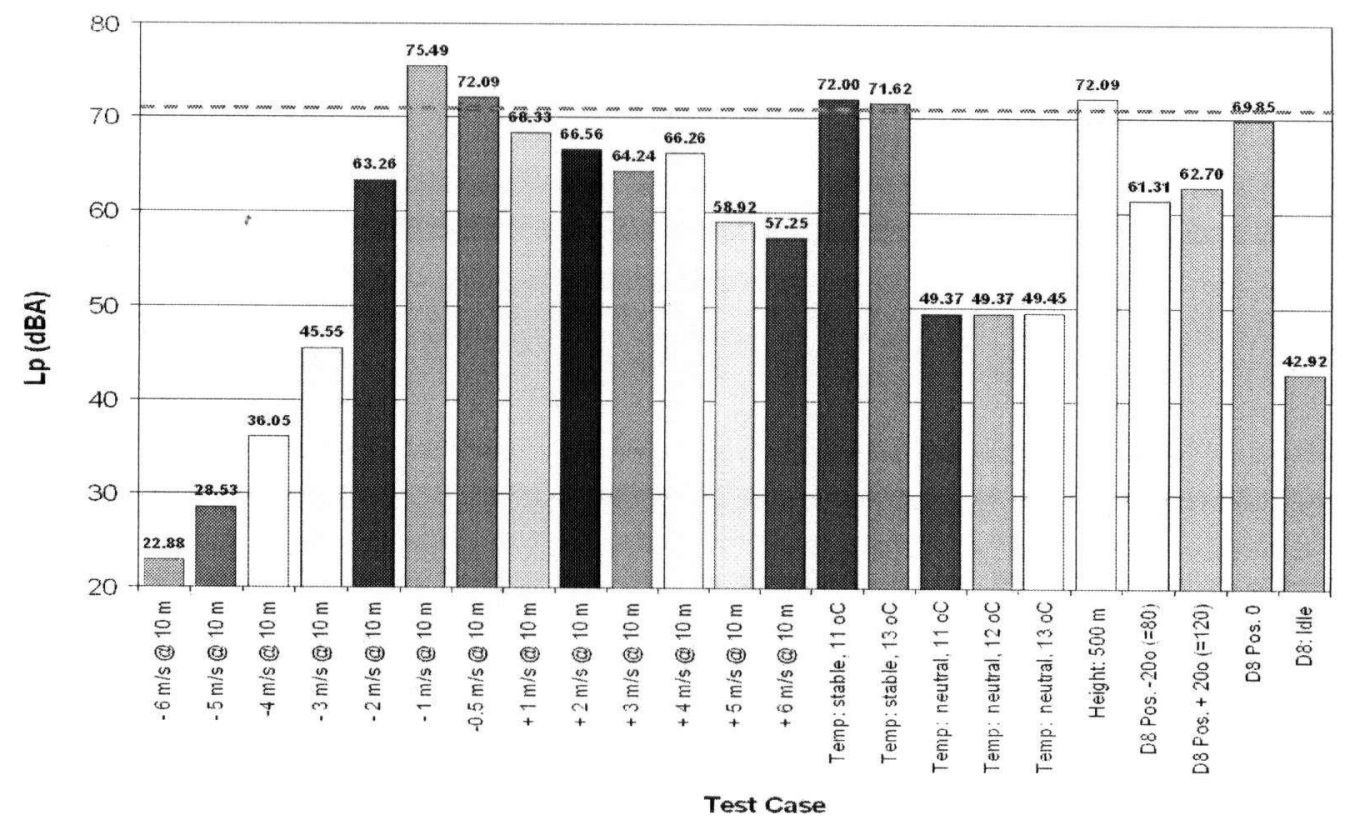

Figure 5.3: Reference test case (71.29 dBA) compared with predictions for input variations in wind, temperature, height of propagation, and aircraft details. (_) is the reference level.

input have very little effect $(<0.5 \mathrm{dBA})$ on the predicted level. What does influence the predicted levels significantly is the state of the atmosphere - specifically, if stable or neutral conditions exist. According to this sensitivity analysis, the difference between these two cases is $22 \mathrm{dBA}$, indicating a major cause of concern - particularly since, without taking temperature measurements up to several tens of meters high, the temperature profile cannot be known for certain.

'Height' in the next column refers to the height above the ground of the atmosphere through which sound is propagating (not including the absorbing layer). In the predictions throughout this work, different atmosphere heights were used. The difference between values calculated for heights of 200 (or $250 \mathrm{~m}$ ), and $500 \mathrm{~m}$ was always, as is the case here, within $\sim 1 \mathrm{~dB}$; this was not believed to be worth the additional computation time associated with increasing the height above the ground for sound propagation.

The last four cases in Figure 5.3 refer to changes in the heading and power level. Aircraft are directional noise sources and the aircraft heading significantly influences the predicted sound pressure levels, as seen in the next three columns. The engine power setting for the 
run-up is equally, if not more, important (see last column): at idle, the predicted sound pressure level $2 \mathrm{~km}$ from a Dash- 8 is $28 \mathrm{dBA}$ quieter than when it is at full power. The importance of how closely the aircraft operators follow the specifications on their run-up request forms cannot be stressed enough: last minute 'minor' modifications to power settings and headings can be the direct cause of sound levels being significantly higher in the local communities, and can thus result in increased run-up noise annoyance.

\subsubsection{Summary}

The sensitivity of the GFPE model with regard to variations in most input parameters is rather robust: realistic variations do not yield very different results. The exceptions to this are in the cases of wind, atmosphere type, and aircraft power setting and heading. The values of the latter two factors are well known and, as long as communication between the aircraft operator and YVR Operations is clear, this information should be readily accessible. Wind profiles and the state of the atmosphere are a different story: these will never be known with absolute certainty unless they are measured. Their dynamic nature makes the feasibility of measurements even more difficult. If there is significant doubt when predicting sound pressure levels in communities, the worst case scenario should be adopted: this is the stable atmosphere.

\subsection{GFPE Run-up Prediction Results}

As described earlier in the chapter, the run-up events chosen for prediction were those with run-up noise complaints correlating with the time of the run-up events. First considered were 22 run-up events that took place over 16 nights. These, along with the corresponding prediction results, are listed in Table 5.1.

Many things are being compared in Table 5.1, such as different general atmospheric states. The first three columns indicate the date and time of each run-up. The next column lists the NMT closest to the location from where the noise complaint came. The next grouping, NMT measurements, lists the background noise, usually prior to the event, the average level, and the peak 1-s Leq level during the event. The event time was taken to be that from the incident report, unless the data clearly showed a peak that resembled a 


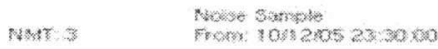
Noxise Sastraque

Fo: $1043 \times 20$ ot 30000

Run-up event

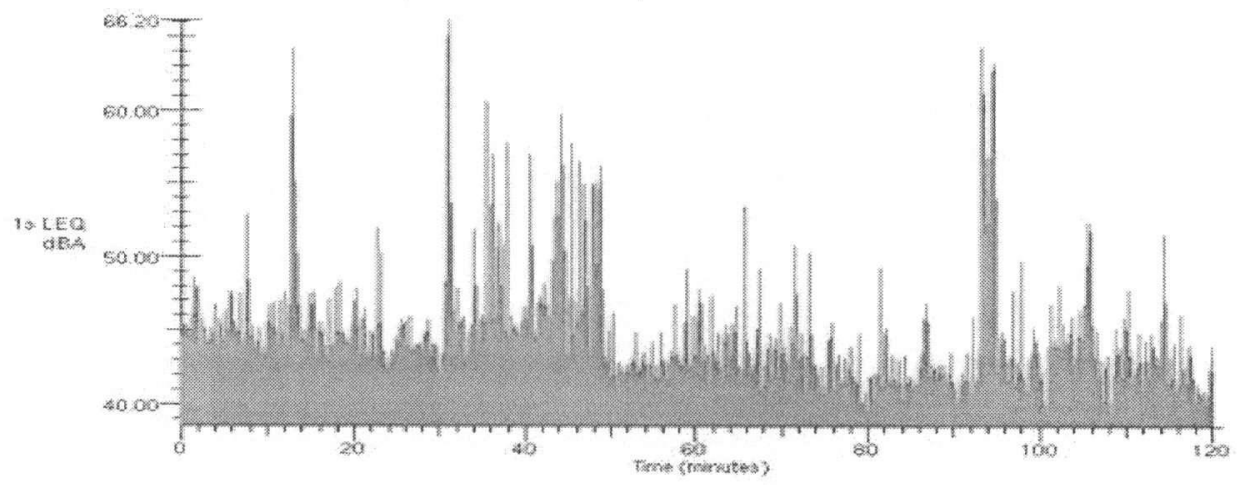

Figure 5.4: Data from NMT 3, October 12, 2005. A noise event is apparent from $\sim$ 0:00 to 0:20 AM. ( 0:30 to 0:50 on the time axis)

run-up noise event a few minutes earlier or later. Background levels were found by taking the 10-min Leq just prior to the event, unless another section of the NMT record clearly appeared to correspond to background levels. Figure 5.4 shows an example of the NMT data available from run-ups.

In Table 5.1, the column GFPE Predictions shows the model predictions obtained using parameters discussed in previous chapters (e.g. horizontal step size of $10 \lambda$, frequency range of $25 \mathrm{~Hz}-4 \mathrm{kHz}$ ). Where some information in the incident reports was missing, a second case was sometimes predicted, as shown in the brackets. SEL was predicted in $d B$ and in $\mathrm{dBA}$ - the former to be used in ANSI's prediction of people awoken, the latter to be compared with the SEL events identified by the NMTs, which only measure dBA. SEL data, as listed in the last seven columns, was obtained from the NMTs: threshold values are set at each terminal; when noise levels surpass this threshold, noise events are recorded. SEL data for run-ups in 2006 was not yet available. GFPE predictions with winds greater than $5 \mathrm{~m} / \mathrm{s}$ have been included in this table. For these predictions, the state of the atmosphere when the winds were below $5 \mathrm{~m} / \mathrm{s}$ was input into the model.

Some GFPE predictions in Table 5.1, primarily those that predict negative values, do not appear to represent the noise sources that caused the noise complaint. From the caller's description of the noise when phoning in the complaint, it is not surprising that this was 
Table 5.1: Summary of run-up measurement and prediction results for events from Jan. '05 to June '06. Values in () are full power levels, with idle levels in the same cell; values in \{\} are at Pos. 0, with Pos. 100 in the same cell; and values in [] are full power at the correct position, with values at idle, but at the incorrect positions, in the same cell. Cells highlighted (ם) represent stable,

represent neutral, and

represent unstable (wind speed

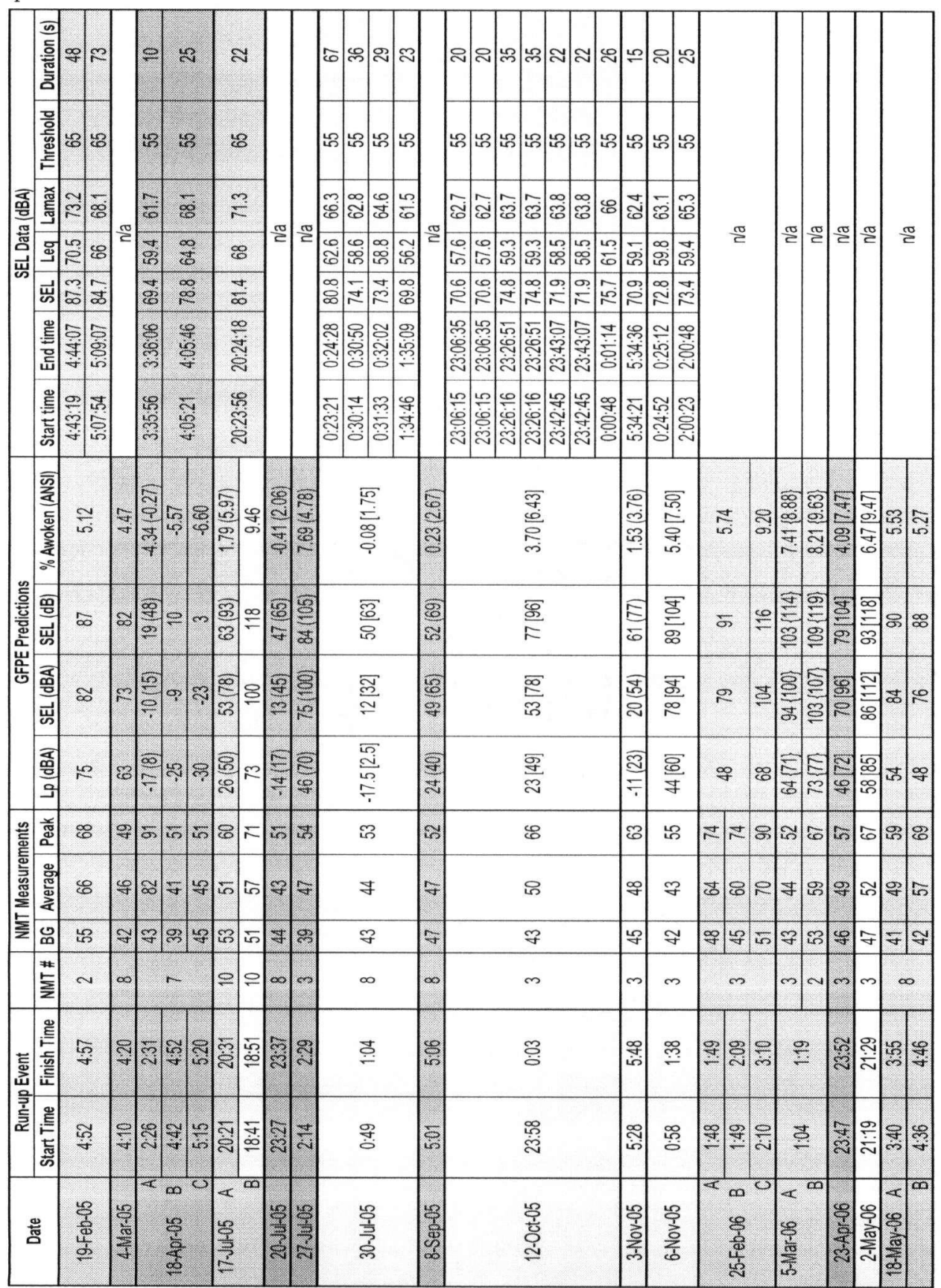




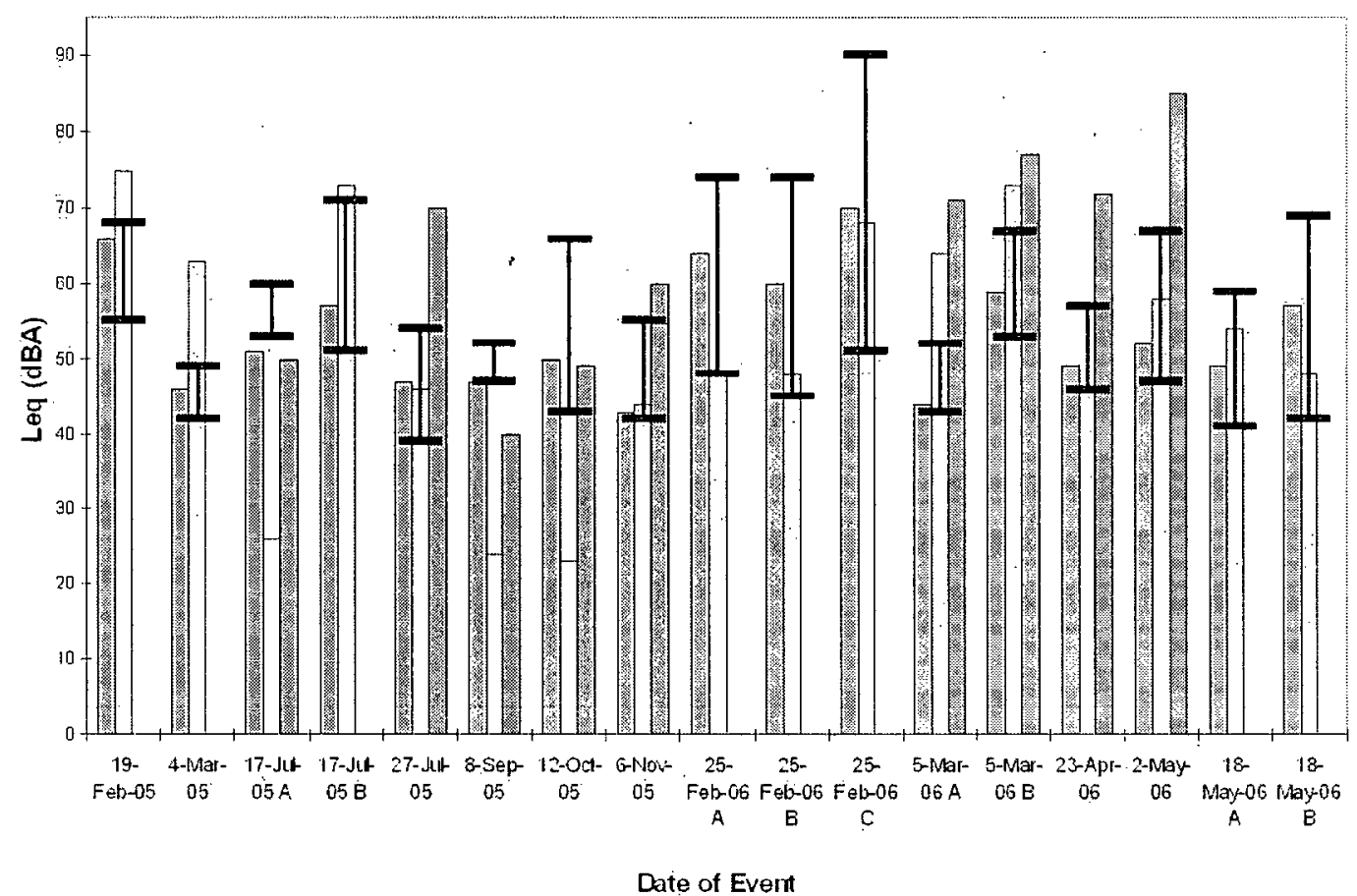

Figure 5.5: Summary of run-up noise levels from GFPE predictions, and estimates from NMT data. (ם) NMT Leq average, (ם) low GFPE prediction, (口) high GFPE prediction; black lines indicate the runge of NMT levels (from background to peak).

sometimes the case. For example, the person phoning on April 18th 2005 said that they were "woken up many times due to high-pitched noises between 2:30 and 5:30 AM".

To understand how well the GFPE predictions fit with measured NMT data, predictions should be compared with measured peak, average, and background levels. This has been done graphically in Figure 5.5, with the negative predictions from Table 5.1 removed. Two GFPE predictions are shown for events for which information was missing, and more than one value of the missing inputs was used. These have been labeled as 'low' and 'high' GFPE predictions - the names distinguish the two predictions, but imply nothing about the range of levels predicted by a GFPE simulation. The prediction that was closest to the average level was used in the discussion.

Due to the wind and temperature profiles, the speed of sound in several cases, particularly in neutral atmospheres, decreased with height. Typically the decrease was $\sim 1 \mathrm{~m} / \mathrm{s}$ from the ground level to $200 \mathrm{~m}$. This change in sound speed leads to very weak upward refraction. To verify if turbulence should be included in these cases, the phase screens from 

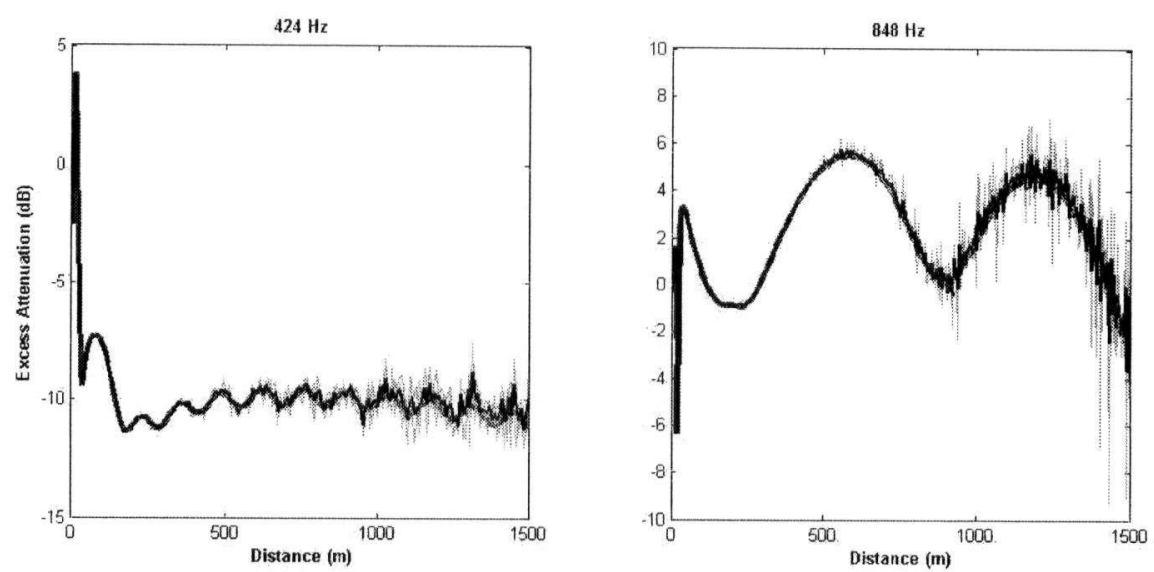

Figure 5.6: GFPE predictions using turbulence for the 8-Sept-05 sound speed profile.

is the non-turbulent prediction, (_ ), ( ), and ( ) correspond to the three predictions with turbulence, and (_-_-) is the $d B$ average of the three turbulent predictions.

Chapter 4 were used with the 8-Sep-05 sound speed profile.

Predictions for 424 and $848 \mathrm{~Hz}$ are shown in Figure 5.6. Fluctuations in the sound pressure level do increase with distance at both frequencies; however the decibel average of these fluctuations is still within $\sim 1 \mathrm{~dB}$ of the non-turbulent case. Predicting sound levels in a turbulent atmosphere is apparently not the cause of differences much greater than a few decibels. This demonstrates that turbulence need not be included in the current run-up predictions.

A discussion of the results in Figure 5.5 follows. Run-up events have been grouped according to available information and to their atmospheric state.

\subsubsection{Available Aircraft Source Levels, Known Headings}

There were three stable cases on two nights for which the correct aircraft source levels were available, the aircraft heading was known, and winds velocities were below $5 \mathrm{~m} / \mathrm{s}$ (in fact, they were all below $4 \mathrm{~m} / \mathrm{s}$ ): on 19-Feb-05, and the two run-ups on 18-May-06 (A and B). In the first two cases, GFPE predictions overestimated the average level by 9 and $5 \mathrm{dBA}$, respectively. This leads to a very interesting situation: toward the beginning of this thesis, it was mentioned that there was a blast fence on one side of Run-up Pad 5. As it turns out, the average insertion loss in each third-octave band was approximately $8 \mathrm{~dB}$ [18]. For 
the geometry of the run-up on 19-Feb-05, the blast fence was between the aircraft and the receiver. Applying the insertion loss estimate from [18], the predicted value for 19-Feb-05 is within $\sim 1 \mathrm{~dB}$ of measurement.

There was one case with a known heading and available aircraft noise data for neutral conditions. Predicted levels for this case, on 8-Sep-05, were $7 \mathrm{dBA}$ below the average measured level. This was the case for which turbulence was included in the prediction earlier (see Figure 5.6), but was found not to effect the prediction by more than a decibel or two.

\subsubsection{Unavailable Aircraft Source Levels, Known Headings}

For the cases when the source data was unavailable for the specific aircraft, but the heading was given, two stable cases (same aircraft, events one after the other the same night) and one neutral case existed with wind speeds below $5 \mathrm{~m} / \mathrm{s}$ : on 25-Feb-06 (A \& B), and on 4Mar-05, respectively. For the stable cases, the GFPE predictions were 12 and $16 \mathrm{dBA}$ below the average levels. The cause of this difference may very well be the aircraft substitution of a Beechcraft 1900 for a Beechcraft $100^{5}$.

The neutral case, on 4-Mar-05, over-predicted levels by $17 \mathrm{dBA}$ relative to the average level. While at first this seems surprising; since the CRJ200 was replacing Boeing 767 levels $^{6}$ here, again, the blast fence at Run-up Pad 5 fell between the source and receiver. Factoring its attenuation into the equation, measured levels are more closely predicted.

\subsubsection{Available Aircraft Source Levels, Unknown Headings}

Determining the aircraft heading (its orientation) when it hasn't been recorded makes predictions difficult, since aircraft are very directional (particularly propeller aircraft). One night's events (on 5-Mar-06 A\&B) predicted levels for NMT 2 and 3 for aircraft for which source levels were available, but the headings were unknown. These predictions were 20

\footnotetext{
${ }^{5}$ Both the 1900 and the 100 are twin engine turboprop aircraft. The 1900 is larger, designed to carry 19 passengers, while the 100 was designed for 12 passengers. The 100 is much older, and production was discontinied more than twenty years ago, shortly after the 1900 came into service. Production of the 1900 has also recently stoppèd.

${ }^{6}$ Takeoff, lateral, and approach levels in EPNdb arc 77.6, 82.4, and 92.1 for the CR.J200, and 93.2, 97.0, and 100.2 for the 767 , respectively $[52,65]$.
} 
and $14 \mathrm{dBA}$ above average levels, for Position 0 (Position 100 over-predicted levels even more). Taking into account the combination of the insertion loss due to the blast fence (this was another run-up that occurred at Run-up Pad 5), and the possibility that the true heading had lower sound pressure levels, this prediction could be much closer to the average measured level than as presented here. March 5, 2006 was also noted to have a stable atmosphere. The positive change in temperature between heights of 1.6 and $6 \mathrm{~m}$ is the largest of all the run-up events considered: $4.3^{\circ} \mathrm{C}$ over $4.4 \mathrm{~m}$. Wind velocities are relatively low, at $1.1 \mathrm{~m} / \mathrm{s}$ at $10 \mathrm{~m}$; the difference between prediction and measurement is not believed to be caused by unknown atmospheric parameters in this case.

\subsubsection{Unavailable Aircraft Source Levels, Unknown Headings}

Four run-up events fall into this category, with unknown aircraft source levels and unknown headings: two under stable conditions, on 17-Jul-05A and on 25-Feb-06C, and two neutral cases, on 27-Jul-05 and on 23-Apr-06. Astonishingly, GFPE predictions under-predicted the average levels in the four cases by only $1,2,1$, and $3 \mathrm{dBA}$, respectively. The event on 17-J111-05 was recorded as an idle event, but when the full power prediction level was used for the source, the best prediction was obtained. The idle power setting listed on 27-Jul-05 agreed very well - better than the full power prediction. This suggests that, sometimes, the aircraft being run-up are at the idle power setting that is recorded, and other times, they are likely at a higher power setting. The 23-Apr-06 prediction gives a level equal to the background noise. What is interesting here is how decibel addition $(\oplus)$ works: ignoring the A-weighting for a moment,

$$
46 \mathrm{~dB} \text { (background level) } \oplus 46 \mathrm{~dB} \text { (prediction level) }=49 \mathrm{~dB} \text {, }
$$

which happens to be the average measured level according to the NMT. The prediction for $25-F e b-06 \mathrm{C}$ is well above the background levels and below the peak level, and only $2 \mathrm{dBA}$ from the average noise event level. It is very surprising how well the levels in this section

agree, given the uncertainty in the input. This clearly won't always be the case, since chance has something to do with choosing the best values for the unknown parameters. 


\subsubsection{Predictions With Winds Above $5 \mathrm{~m} / \mathrm{s}$}

Four run-ups remain to be discussed, each having occurred during high wind conditions: on 17-Jul-05B, 2-May-06, 12-Oct-05, and 6-Nov-05. If wind velocities were smaller, the former two would have been categorized as stable, and the latter two as neutral. If stable and neutral conditions are assumed, respectively, predictions obtained with the GFPE model are $+16,+6,-1$, and $+1 \mathrm{dBA}$ different from the average measured levels of the noise events, respectively. Earlier in this chapter it was shown that the GFPE model is rather sensitive to higher winds - particularly upwind. Wind should not affect the propagation of sound significantly for any of these cases because the source-receiver direction is never parallel to the wind direction for any of the four events (the differences between the source-receiver directions and the wind directions for each case are $55^{\circ}, 124^{\circ}, 74^{\circ}$, and $135^{\circ}$, respectively). These stronger winds are, however, expected to affect the atmosphere, preventing the 'standard' temperature profiles (parabolic or linear) from existing at higher heights, or even becoming established.

What makes this analysis extremely difficult is that information pertaining to the aircraft source levels and/or the aircraft heading is missing for all four run-up events, except on 6-Nov-05 (which was over-predicted by only $1 \mathrm{dBA}$ ). While, theoretically, strong winds should affect the atmosphere such as to make predictions difficult, uncertainties corresponding to the aircraft make this hard to isolate. The fact that more than one GFPE prediction was made for all, events except 17-Jul-05, increases to the chance of finding one prediction that agrees with the average measured level well.

\subsubsection{Summary}

Predictions, corrected for insertion loss where applicable, have been plotted again in Figure 5.7. The many points near to the $y=x$ line suggest that the GFPE model is predicting sound pressure levels with reasonable accuracy to the measured levels. The scatter indicates the GFPE model doesn't have a tendency to over- or under-predict sound levels. In the end, a discussion comparing peak measurement values (as shown by the upper error bars in Figure 5.7) and GFPE predictions was omitted, as there are a plethora of possible explanations for the cause of peak levels (e.g. large trucks driving by, overhead aircraft, car 
alarms, dogs barking, etc.). The cause of the peak may not even be that obvious, since the peak level only needs to last one second to be registered.

The input parameters to which the GFPE model was most sensitive were also the parameters that ended up being most difficult to obtain correctly and with certainty. While many $(71 \%)$ of the event average levels in Figure 5.5 - once corrected for insertion loss, where applicable - were predicted within $10 \mathrm{dBA}$, some events were inaccurately predicted by nearly $20 \mathrm{dBA}$ : the worst prediction was for the Boeing 767 on March 4, 2005, which was 17 dBA higher than the average NMT level. The results from Figure 5.5 - again corrected for insertion loss, where applicable - have been split into those that were within, and those

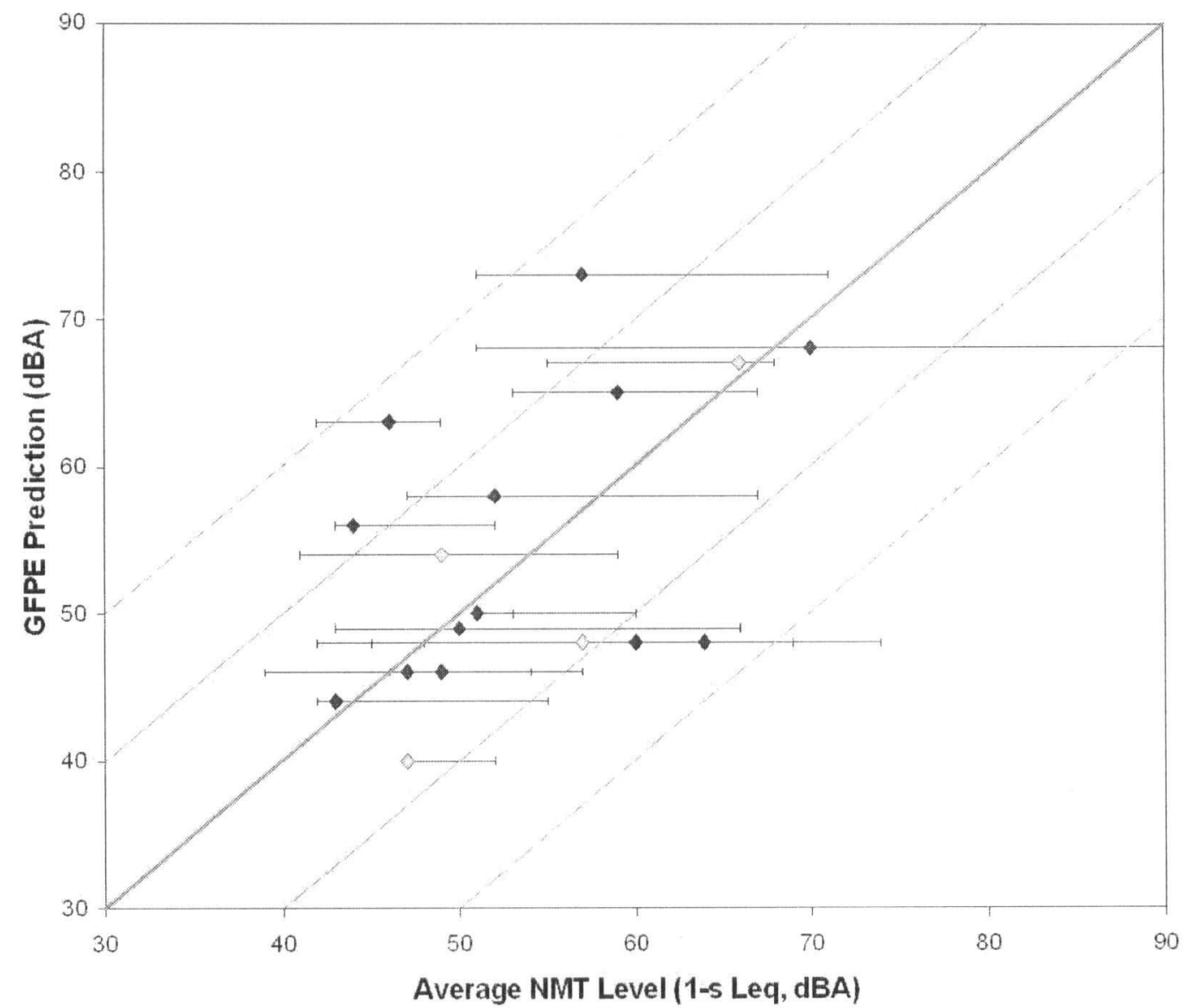

Figure 5.7: Scatter plot comparing GFPE predictions with NMT measurements. ( ) represents $y=x$, and 10 and $20 \mathrm{dBA}$ offsets, ( ) are the GFPE predictions where some information was unknown, and are the GFPE predictions where all information was known. Error bars represent the background and peak NMT measurement levels. 
Table 5.2: Summary of run-up event categories, separated into those within $10 \mathrm{dBA}$ of the average, and those for which predictions were greater than $10 \mathrm{dBA}$ of the average.

\begin{tabular}{|c|c|c|}
\hline Case & $\begin{array}{c}\text { Number of predictions } \\
\leq 10 \mathrm{dBA}(\%, \text { out of } 12)\end{array}$ & $\begin{array}{c}\text { Number of predictions } \\
>10 \mathrm{dBA}(\%, \text { out of } 5)\end{array}$ \\
\hline Available aircraft levels & $7(58)$ & $1(20)$ \\
\hline Known heading & $6(50)$ & $4(80)$ \\
\hline Wind less than $5 \mathrm{~m} / \mathrm{s}$ & $9(75)$ & $4(80)$ \\
\hline Stable (regardless of wind speed) & $7(58)$ & $4(80)$ \\
\hline Greater than average level & $5(42)$ & $3(60)$ \\
\hline
\end{tabular}

that were greater than, $10 \mathrm{dBA}$ with respect to the average levels in Table 5.2.

With this smaller sample size, and the surprisingly close predictions compared with average levels in Sections 5.3.4 and 5.3.5, it is hard to draw conclusions in regards to what conditions are more likely to result in inaccurate predictions; however Table 5.2 attempts to do so. It appears, although the sample size is rather small, that for run-up events for which the aircraft noise levels are available, there is nearly three times as good of a chance that the prediction will be within $10 \mathrm{dBA}$ than if the levels were estimated with another aircraft. It is more difficult to draw conclusions from the next four cases, which deal with whether the heading is known, if wind speeds are less than $5 \mathrm{~m} / \mathrm{s}$, if the atmosphere is stable (an increase in temperature from the $1.6 \mathrm{~m}$ to $6 \mathrm{~m}$ height, regardless of wind speed), as they give similar results. The final case, whether predictions tend to be above or below the average levels, was interesting: overestimates appear to be equally as likely to occur as underestimates of the average level. Again, with this small sample size, and the surprising predictions in Sections 5.3.4 and 5.3.5, it difficult to have confidence in the results in Table 5.2 .

According to the results in Section 5.3.1, if all information is available, particularly the noise levels of the aircraft performing the run-up, prediction can be expected to be within approximately $10 \mathrm{dBA}$. With relevant information unknown, prediction uncertainty will increase. With careful choices, however, predictions can be expected to be within approximately $20 \mathrm{dBA}$. 
Table 5.3: Summary of SEL data corresponding to SEL GFPE predictions

\begin{tabular}{|c|c|c|c|c|c|c|c|c|c|c|c|c|}
\hline \multirow{2}{*}{ Date } & \multicolumn{2}{|c|}{ Run-up Event } & \multicolumn{3}{|c|}{ GFPE Predictions } & \multicolumn{7}{|c|}{ SEL Data } \\
\hline & $\begin{array}{l}\text { Start } \\
\text { Time }\end{array}$ & $\begin{array}{c}\text { Finish } \\
\text { Time }\end{array}$ & $\begin{array}{c}\mathrm{Lp} \\
\text { (dBA) }\end{array}$ & $\begin{array}{c}\text { SEL } \\
(\mathrm{dBA})\end{array}$ & $\begin{array}{c}\text { Duration } \\
(s)\end{array}$ & $\begin{array}{l}\text { Start } \\
\text { time }\end{array}$ & $\begin{array}{l}\text { End } \\
\text { time }\end{array}$ & Leq (dBA) & SSEL (dBA) & Lamax & $\begin{array}{c}\text { Threshold } \\
\text { Level } \\
\text { (dBA) }\end{array}$ & $\begin{array}{c}\text { Duration } \\
\text { (5) }\end{array}$ \\
\hline 17-Jul=0.5 A & $20: 21$ & $20: 31$ & $28(50)$ & $53(76)$ & 600 & 20:23:56 & $20: 24: 18$ & 68 & 81.4 & 71.3 & 65 & 22 \\
\hline $12-0 c t-05$ & $23: 58$ & 003 & $23[49]$ & $53[78]$ & 300 & $0: 00: 48$ & $001: 14$ & 61.5 & 75.7 & 66 & 55 & 26 \\
\hline
\end{tabular}

\subsection{SEL and Annoyance}

SEL levels recorded by NMTs were listed in Table 5.1, to verify how well they identified the events predicted by the GFPE model's $S E L$ output. In general, the match was quite poor: only two run-up events had $S E L$ events occurring at the same time (on 17-Jul-05A and 12-Oct-05). While the SELs were quite close, the 1-s equivalent Leq from the SEL data was much higher, and the events much shorter, than those predicted with the GFPE model. These results have been summarized in Table 5.3 .

Using only the predictions in Figure 5.5, the percentage of people annoyed due to the engine run-ups identified in this work ranges from 1.8 to $9.5 \%$, with the average being $5.9 \%$. An uncertainty of $\pm 10 \mathrm{~dB}$ in $S E L$ level corresponds to an uncertainty of $\pm 1.4 \% ; \pm 20 \mathrm{~dB}$ results in $\pm 2.8 \%$. Remember also that the $S E L$ values predicted here are outdoor levels. If indoor levels were available and used, as they should be according to ANSI S12.9-2000/Part 6, SEL would be lower; thus the true percentage of people awoken is likely lower.

While light sleepers may be very sensitive to noise, and be awoken regardless of the $S E L$, to lower this percentage theoretically to zero, $S E L$ should be below $50 \mathrm{~dB}$. Exceptions will always exist; depending on the geometry of neighbourhoods and of houses, some sound focusing probably takes place, making some areas louder than others. Even if predictions of $S E L$ are well below $50 \mathrm{~dB}$, there is a chance that some people will be awoken.

The following chapter concludes this thesis. The work and results are summarized, and suggestions for future work follow. 


\section{Chapter 6}

\section{Conclusion}

\subsection{Summary}

The final objective of this work was to create a novel prediction tool that would aid the Vancouver Airport Authority in planning aircraft engine run-ups, to minimize noise levels, and thus community disturbance, for the residents of the community living near the airport. This objective has been achieved.

In order to attain this final objective, various parameters that influence the propagation of sound were understood, and incorporated into a simple outdoor sound propagation model. The original GFPE model that was available when this work began was simplistic modifications were necessary to better approximate the atmosphere, and also to produce a more practical output. The latter included multiple frequencies, air absorption, and overall A-weighted levels that were computed using frequency-dependent step sizes. The modifications to better approximate the propagation through the atmosphere were for wind and temperature gradients, mixed ground impedance, and atmospheric turbulence.

To ensure changes made to the GFPE code were accurate, the model was evaluated. A comparison of predictions with those in the literature found very good agreement; typically the two levels were within $2 \mathrm{~dB}$ of each other. Since someone with good hearing can only just make out a $1 \mathrm{~dB}$ difference, especially for distances out to several kilometers, the agreement with literature was considered to be sufficiently accurate to use the GFPE model in real-life predictions.

To test the GFPE code, a selection of run-ups that occurred between January 2005 and June 2006 were drawn upon. In order for a run-up to be considered, someone in the community, whose address or approximate location was available, was required to have reported a noise complaint to YVRAA corresponding to the approximate time of an engine run-up. Only nighttime run-up events were included. Seventeen such events were identified, and their resulting sound pressure levels and annoyance were predicted and discussed. 
Availability of input information turned out to be a large problem: a sensitivity analysis found that the state of the atmosphere, wind speeds, and aircraft power levels and headings were the major sources of variability to the outputs of the model. Each of these parameters was difficult to obtain with certainty, and estimates of unknown input data were required in several cases. A-weighted prediction levels for cases for which all input information was available were within $10 \mathrm{dBA}$ of the average level recorded at the corresponding noise monitoring terminal. For cases where some information (i.e. the aircraft heading) was missing, the uncertainty of the run-up predictions were within $20 \mathrm{dBA}$.

One more step was required to obtain the final objective: a prediction of the community annoyance caused by noise. ANSI Standard S12.9-200/Part 6, which relates sound exposure level to the percentage of people awoken, gives a simple relationship, which was used. According to the sound pressure levels predicted in the communities for this seventeen run-up event sample set, the corresponding percentage of people awoken ranged from 1.8 to $9.5 \%$. This method of identifying annoyance is likely a better way to assess the number of people disturbed by noise than are complaints, as the entire population will not feel inclined to report incidents, even if their sleep is disturbed.

While uncertainties of $\pm 10 \mathrm{dBA}$ (and $20 \mathrm{dBA}$ ) are rather large, given the complexity of the problem, the agreement, was extremely good. Almost all prediction parameters - the ground impedance, the temperature and wind profiles, the (negligible) atmospheric turbulence, the source levels, the source and receiver positions, the air absorption - had to be estimated or approximated in some way. More information - particularly relating to the atmosphere - is crucial to obtaining more reliable predictions.

Parabolic equation models for predicting outdoor sound propagation are popular, as they have the potential to incorporate many effects. The Green's function solution is a method of solving the wave equation fast; for these reasons the GFPE was the best choice for this work. In the end, the GFPE model was found to be rather robust, accurate, and relatively quick at predicting sound levels. It is not at the point where real-time predictions can be done, but on a standard desktop computer, final levels are usually obtained in a couple of minutes. 


\subsection{Contribution to the field}

The work accomplished in this thesis was unique in that outdoor acoustic theory, numerical methods, and noise measurements were combined with human perception of annoyance, to create a tool that can help solve a very real problem in our community: the cause of engine run-up noise complaints (aircraft, heading, atmospheric conditions, etc.), and the magnitude of the disturbance. Many groups of people - the Vancouver Airport Authority, the airlines, the mechanics and pilots repairing and testing the aircraft, and of course the residents of the local communities - are involved in this run-up noise problem. The best results can only be achieved when all of these groups work together; through feedback and careful documentation of both run-up and noise-complaint events, the problems and concerns can be identified and resolved. This thesis has created, validated, and applied a tool which can bring together the information contributed by each party and which can, through prediction, identify problematic locations, aircraft, and general atmospheric conditions, and quantify the noise levels responsible for annoyance.

\subsection{Future Work}

Predictions can be used immediately to compute sound pressure levels and annoyance in communities; however, this project is currently at the stage which makes a good starting point for future work. There are two major aspects that should be addressed and investigated in further work. The first, as suggested throughout the thesis, is related to the atmosphere. Wind profiles, temperatures profiles, and turbulence all have the potential to significantly alter the level of noise propagating outdoors. The best approximations possible with the current body of knowledge have been made in this thesis; however, it would be very interesting to look into this further - perhaps by measuring the temperature and wind profiles during a run-up, and using measured fluctuations in wind and temperature to predict turbulence.

The second issue involves a more creative endeavor. While the current GFPE model can predict noise levels and annoyance, the user must have a good understanding of what they are predicting, and what inputs they should be using. It could be very useful to modify the GFPE code further, such that it is more automated (i.e. the user only has to indicate the 
source and receiver positions, and all distances, including transitions from one ground type to another, are automatically calculated), and predicts sound pressure levels in more than one direction in a single simulation, or even, extend the 2D GFPE model to 3D. For instance, one extension could allow the user to choose three possible run-up pads: the GFPE model would calculate sound pressure levels in the key problematic communities, and the location and heading resulting in the least amount of disturbance would be identified for the particular aircraft. With the optimal heading and location known for the run-up of a certain type of aircraft and given atmospheric conditions, YVR Operations would be able to give permission to aircraft operators for run-ups, knowing that they have the best configuration possible to allow airlines to conduct business as required, while maintaining good relationships with the local community.

To ensure run-up noise is being modeled properly for all aircraft, it would also be beneficial to measure the noise characteristics of at least one larger jet aircraft, as the noise characteristics are expected to be different from the propeller and small jet aircraft previously measured. In an ideal world, the noise characteristics of all aircraft operating and performing run-ups at YVR would be measured. Daytime run-ups and curved ground could also be considered in future work.

With this prediction model now operational, many new avenues for study and implementation are available. Future work, in addition to the ideas described above, could include a study on the transmission loss in houses, or annoyance in forms other than sleep disturbance. Outdoor sound propagation is a complex phenomena; this thesis project has attempted to improve our ability to model its fundamental properties and predict its effects. Further work in this field will continue to promote a harmonious relationship between the Vancouver Airport and surrounding communities. 


\section{Bibliography}

[1] K. Attenborough; Personal communication; 2005.

[2] E.M. Salomons. Computational Atmospheric Acoustics. Kluwer Academic Publishers, Dordrecht, The Netherlands, 2001.

[3] F. Tewfik. Presentation, Noise Management: The Sound of a Gateway. 2004 Noise Management Training. YVRAA, 2004.

[4] The Vancouver International Airport Authority. Airport operations directives section: Noise abatement, Subject: Engine run-ups. Document, number 02-06-0100. 2003.

[5] Vancouver International Airport home page (http://www.yvr.ca/) 2006.

[6] T.F.W. Embleton. Tutorial on sound propagation outdoors. Journal of the Acoustical Society of America, 100:31-48, 1996.

[7] A. Muradali and K.R. Fyfe. Accurate barrier modeling in the presence of atmospheric effects. Applied Acoustics, 56:157-182, 1999.

[8] G.A. Daigle, J.E. Piercy, and T.F.W. Embleton. Effects of atmospheric turbulence on the interference of sound waves near a hard boundary. Journal of the Acoustical Society of America, 64:622-630, 1978.

[9] K.E. Gilbert, R. Raspet, and X. Di. Calculation of turbulence effects in an upwardrefracting atmosphere. Journal of the Acoustical Society of America, 87:2428-2437, 1990.

[10] Federal Interagency Committee on Aviation Noise website (www.fican.org) 2006.

[11] Federal Interagency Committee on Aviation Noise (FICAN) website (http : //www.fican.org/pdf/Effects viationNoise $_{S}$ leep.pdf). Effect of aviation noise on awakenings from sleep. 2006. 
[12] ANSI S12.9-2000/Part 6: American National Standard Quantities, Procedures for Description, and Measurement of Environmental Sound Part 6: Methods for Estimation of Awakenings Associated with Aircraft Noise Events Heard in Homes, 2005.

[13] A. Nakashima. Active control of propeller aircraft run-up noise. M.A.Sc. Thesis 2003.

[14] M. West, K. Gilbert, and R.A. Sack. A tutorial on the parabolic equation (pe) model used for long range sound propagation in the atmsophere. Applied Acoustics, 37:31-49, 1992.

[15] K. Attenborough, S. Taherzadeh, H.E. Bass, X. Di, R. Raspet, G.R. Becker, A. Gudesen, A. Chrestman, G.A. Daigle, A. L'Espérance, Y. Gabillet, K.E. Gilbert, Y.L. Li, M.J. White, P. Naz, J.M. Noble, and H.A.J.M. van Hoof. Benchmark cases for outdoor sound propagation models. Journal of the Acoustical Society of America, 97:173-191, 1995.

[16] B. Gauvreau, M. Bérengier, P Blanc-Benon, and C. Depollier. Traffic noise pediction with the parabolic equation method: Validation of a split-step padé approach in complex environments. Journal of the Acoustical Society of America, 112:2680-2687, 2002.

[17] G.A. Daigle, J. Nicolas, and J.-L. Berry. Propagation of noise above ground having an impedance discontinuity. Journal of the Acoustical Society of America, 77:127-138, 1985.

[18] P. Germain. Active control of run-up noise from propeller aircraft. M.A.Sc. Thesis 2000.

[19] K. Attenborough. Sound propagation close to the ground. Annual Review of Fluid Mechanics, 34:51-82, 2002.

[20] A. Nakashima and M. Hodgson. Effect of realistic grounds and atmospheric conditions on single-channel active control of outdoor sound propagation, Part 1. Journal of the Acoustical Society of America, 117:1080 to 1087, 2005.

[21] O. Zaporozhets, V. Tokarev, and K. Attenborough. Predicting noise from aircraft operated on the ground. Applied Acoustics, 64:941-953, 2003. 
[22] T.F.W. Embleton, J.E. Piercy, and N. Olson. Outdoor sound propagation over ground of finite impedance. Journal of the Acoustical Society of America, 59:267-277, 1976.

[23] M. Hodgson. Course notes from Fall 2005 Term Mech 584: Advanced Engineering Acoustics; University of British Columbia.

[24] A.J. Zuckerward. Acoustic ground impedance meter. Journal of the Acoustical Society of America, 73:2180-2186, 1983.

[25] G.A. Daigle, T.F.W. Embleton, and J.E. Piercy. Experiments on surface waves over a model impedance plane using acoustical pulses. Journal of the Acoustical Society of America, 99:1993-2005, 1996.

[26] M.E. Delany and E.N. Bazley. Acoustical properties of fibrous materials. Applied Acoustics, 3:105-116, 1970.

[27] T.F.W. Embleton, J.E. Piercy, and G.A. Daigle. Effective flow resistivity of grund surfaces determined by acoustical measurements. Journal of the Acoustical Society of America, 74:1239-1244, 1983.

[28] K. Attenborough. Acoustical impedance models for outdoor gorund surfaces. Journal of Sound and Vibration, 99:521-544, 1985.

[29] K. Attenborough. Ground parameter information for propagation modeling. Journal of the Acoustical Society of America, 92:418-427, 1992.

[30] M. Hodgson. Course notes from Winter Term 2005 Mech 405: Acoustics and Noise Control; University of British Columbia.

[31] M.A. Price, K. Attenborough, and N.W. Heap. Sound attenuation through trees: measurements and models. Journal of the Acoustical Society of America, 84:1836$1844,1988$.

[32] N. Barrier and Y. Gabillet. Traffic noise control by forests: the meteorological screening effect. Proceedings, Euro-Noise 1998, Munich.

[33] P. Chevret, Ph. Blanc-Benon, and D. Juv. A numerical model for sound propagation through a turbulent atmosphere near the ground. Journal of the Acoustical Society of America, 100:3587-3599, 1996. 
[34] V.E. Ostashev, E.M. Salomons, D.K. Wilson, P. Blanc-Benon, and D. Juvé. Sound propagation in a turbulent atmosphere near the ground: A parabolic equation approach. Journal of the Acoustical Society of America, 109:1894-1908, 2001.

[35] U.S. Environmental Protection Agency: World Trade Center (WTC) Research (http : //www.epa.gov/asmdnerl/FineScale/wtc.html) 2006.

[36] M. Stinson; Personal communication; 2006.

[37] J. Martin. Simulation of wave propagatio in random media: theory and applications, pages 463-486. SPIE - the International nSociety for Optical Engineering and the Institue of Physics Publishing, Bellingham, Bristol and Philadelphia, 1992.

[38] X. Di; Personal communication; 2006.

[39] A. Alexandre. An assessment of certain causal models used in surveys on aircraft noise annoyance. Journal of Sound and Vibration, 44:119-125, 1976.

[10] R. Rylander, S. Sorensen, and A. Kajland. Annoyance reactions from aircraft noise exposure. Journal of Sound and Vibration, 24:419-444, 1972.

[41] K. Llume, M. Gregg, C. Thomas, and D. Terranova. Complaints caused by aircraft, operations: an assessment of annoyance by noise level and time of day. Journal of Air Transport Management, 9:153-160, 2003.

[42] R.F.S. Job. The influence of subjective reactions to noise on health effects of the noise. Environment International, 22:93-104, 1996.

[43] S.A. Stansfeld and P. Lercher. Non-auditory physiological effects of noise: five year review and future directions. The 8th International Congress on Noise as a Public Health Problem. Rotterdam, The Netherlands, 2003.

[44] T. Matsui, T. Matsuno, K. Ashimine, K. Hiramatsu, Y. Osada, and T. Yamamoto. The okinawa study: effect of chronic aircraft noise exposure on birth weight, prematurity and intrauterine growth retardation. The 8th International Congress on Noise as a Public Health Problem. Rotterdam, The Netherlands, 2003.

[45] J. Banatvala. Unhealthy airports. The Lancet, 364, Issue 9435, 2004. 
[46] K.S. Pearsons, D.S. Barber, B.G. Tabacnick, and S. Fidell. Predicting noise-induced sleep disturbance. Journal of the Acoustical Society of America, 97:331-338, 1995.

[47] W. Passchier-Vermeer, H.M.E. Miedema, and H. Vos. Aircraft noise and sleep in the Netherlands. The 8th International Congress on Noise as a Public Health Problem. Rotterdam, The Netherlands, 2003.

[48] J.S. Bradley. IBANA-Calc validation studies (for NRC), IRC-RR-125, 2002.

[49] A. Garcia, L.J. Faus, and A.M. Garcia. The community response to aircraft noise around six spanish airports. Journal of Sound and Vibration, 164:45-52, 1993.

[50] R.R.A.M Mato and T.S. Mufuruki. Noise pollution associated with the operation of the dar es salaam international airport. Transportation Research Part D: Transport and Environment, 4:81-89, 1999.

[51] A.C. Pietrasanta. Noise measurement around some jet aircraft. Journal of the Acoustical Society of America, 28:434-442, 1956.

[52] Bombardier Regional Airraft CRJ200 website (http : //www.crj.bombardier.com/CRJ/en/specifications.jsp?langId = en \& $\operatorname{crj} I d=$ 200) 2006.

[53] Downsview Ontario Canada. (Revised November 7 2003) Canada Regional Jet Airport Planning Manual. Issue 7, Model CL-600-2B19 Series 100 / 200 / 440. CSP A-020. Airplane Characteristics for Airport Planning. (c) 2000 Bombardier Inc.

[54] International Organization for Standardization. Acoustics - Procedure for describing aircraft noise heard on the ground. Corrected and reprinted 1981-10-30, 1981.

[55] B. Magliozzi, B. Hanson, and R.K. Amiet. Propeller and Propfan Noise, pages 1-61. Acoustical Society of America, 500 Sunnyside Boulevard; Woodbury, NY, 1995.

[56] E.M. Salomons. Improved green's function parabolic equation method for atmospheric sound propagation. Journal of the Acoustical Society of America, 104:100-111, 1998.

[57] K.E. Gilbert and X. Di. A fast green's function method for one-way sound propagation in the atmosphere. Journal of the Acoustical Society of America, 94:2343-2352, 1993. 
[58] Y.A. Melnikov. Influence functions and matrices. Marcel Dekker, Inc., New York, NY, 1999.

[59] Klohn-Crippen Consultants Ltd. West Apron Expansion Project - Environmental Soil Sampling Final Report. Prepared for the Vancouver ,International Airport Authority, 1999.

[60] G.A. Daigle, T.F.W. Embleton, and J.E. Piercy. Propagation of sound in the presence of gradients and turbulence near the ground. Journal of the Acoustical Society of America, 79:613-627, 1986.

[61] G.S.K. Wong and T.F.W. Embleton. Variation of the speed of sound in air with humidity and temperature. Journal of the Acoustical Society of America, 77:1710$1712,1985$.

[62] J.S. Robertson, P.J. Schlatter, and W.L. Siegmann. Sound propagation over impedance discontinuities with the parabolic equation. Journal of the Acoustical Society of America, 99:761-767, 1996.

[63] F.M. Weiner and D.N. Keast. Experimental study of the propagation of sound over ground. Journal of the Acoustical Society of America, 31:724-733, 1959.

[64] D. Steyn; Personal communication; 2006.

[65] International requirements regarding aircraft noise. website (http : //www.adv net.org/end/drv/umlaerm $30 . p h p)$ ADV (c) 2006. 
In presenting this thesis in partial fulfilment of the requirements for an advanced degree at the University of British Columbia, I agree that the Library shall make it freely available for reference and study. I further agree that permission for extensive copying of this thesis for scholarly purposes may be granted by the head of my department or by his or her representatives. It is understood that copying or publication of this thesis for financial gain shall not be allowed without my written permission.

(Signature)

Department of Mechanical Engineering

The University of British Columbia

Vancouver, Canada

Date 